\title{
Discovery of a Pyrimidothiazolodiazepinone as a potent and selective Focal Adhesion Kinase (FAK) Inhibitor
}

Brian J. Groendyke ${ }^{\dagger, *}, \|$, Behnam Nabet ${ }^{\dagger, \star}, \|$, Mikaela L. Mohardt $\dagger$, Haisheng Zhang ${ }^{\S, \#}$, Ke Peng ${ }^{\#}$, Eriko Koide $\dagger$, Calvin R. Coffey $\dagger$, Jianwei Che ${ }^{\dagger, \ddagger}$, David A. Scott ${ }^{\dagger, \ddagger}$, Adam J. Bass ${ }^{\sharp, \diamond}$, Nathanael S. Gray $^{* \dagger, *}$

${ }^{\dagger}$ Department of Cancer Biology, Dana-Farber Cancer Institute, Boston, Massachusetts, 02115, United States; $\$$ Department of Biological Chemistry and Molecular Pharmacology and Harvard Medical School, Boston, Massachusetts, 02115, United States; §Department of General Surgery, Nanfang Hospital, Southern Medical University, Guangzhou, China; \#Division of Molecular and Cellular Oncology, Dana-Farber Cancer Institute, Harvard Medical School, Boston, Massachusetts, 02115, United States; $\diamond$ Broad Institute of MIT and Harvard, Cambridge, Massachusetts, 02142, United States.

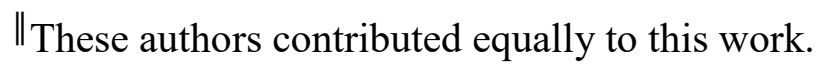

* Correspondence may be addressed to: Nathanael S. Gray (nathanael_gray@dfci.harvard.edu)

\section{Table of Contents}

Pages 2 - 17: KINOMEscan Profiling Supporting Tables and Figures

Pages 18 - 19: Biochemical $\mathrm{IC}_{50}$ Supporting Data

Pages 20 - 23: Microsome Stability and PK Supporting Data

Pages 24 - 25: Kinetic Solubility, Thermodynamic Solubility, and Caco-2 Permeability Assay 
Pages 25 - 27: Docking Analysis of Additional Compounds 3, 20, 26, 37, \& 38

Pages 28 - 29: General Chemistry Methods

Pages 29 - 90: Experimental Procedures and Characterizations

Pages 91 - 94: Biological Methods

Pages 95 - 100: Cell line Supporting Figures

Page 101: Organoid Supporting Figure

Pages $102-103$ : References

$\underline{\text { KINOMEscan Profiling Supporting Tables and Figures }}$

Supporting Table 1. KINOMEscan Profiling of 1 (BJG-01-181) and 4 (BJG-03-025).

\begin{tabular}{|c|c|c|c|}
\hline Structure & $\mathrm{CH}_{3}$ & $\mathrm{CH}_{3}$ & $\mathrm{C}_{3}$ \\
\hline Lab ID & BJG-01-181 & BJG-03-025 & BJG-03-025 \\
\hline GRAY ID & Gray1083 & Gray1142 & Gray 1142 \\
\hline Compound Concentration $(\mu \mathrm{M})$ & 10 & 10 & 1 \\
\hline AAK1 & 48 & 98 & 100 \\
\hline ABL1(E255K)-phosphorylated & 17 & 100 & 100 \\
\hline ABL1(F317I)-nonphosphorylated & 3.8 & 48 & 100 \\
\hline ABL1(F317I)-phosphorylated & 14 & 26 & 67 \\
\hline ABL1(F317L)-nonphosphorylated & 3.9 & 54 & 100 \\
\hline ABL1(F317L)-phosphorylated & 10 & 39 & 93 \\
\hline ABL1(H396P)-nonphosphorylated & 4.3 & 100 & 100 \\
\hline ABL1(H396P)-phosphorylated & 19 & 100 & 100 \\
\hline ABL1(M351T)-phosphorylated & 19 & 95 & 95 \\
\hline ABL1(Q252H)-nonphosphorylated & 7.8 & 93 & 100 \\
\hline ABL1(Q252H)-phosphorylated & 9.7 & 100 & 100 \\
\hline ABL1(T315I)-nonphosphorylated & 100 & 100 & 100 \\
\hline ABL1(T315I)-phosphorylated & 93 & 98 & 83 \\
\hline ABL1(Y253F)-phosphorylated & 47 & 100 & 100 \\
\hline
\end{tabular}




\begin{tabular}{|c|c|c|c|}
\hline ABL1-nonphosphorylated & 25 & 77 & 100 \\
\hline ABL1-phosphorylated & 17 & 79 & 100 \\
\hline ABL2 & 69 & 95 & 100 \\
\hline ACVR1 & 93 & 93 & 100 \\
\hline ACVR1B & 80 & 95 & 100 \\
\hline ACVR2A & 76 & 97 & 100 \\
\hline ACVR2B & 92 & 100 & 100 \\
\hline ACVRL1 & 96 & 99 & 100 \\
\hline ADCK3 & 54 & 88 & 100 \\
\hline ADCK4 & 83 & 73 & 99 \\
\hline AKT1 & 89 & 100 & 100 \\
\hline AKT2 & 75 & 100 & 100 \\
\hline AKT3 & 100 & 100 & 96 \\
\hline ALK & 100 & 99 & 97 \\
\hline $\operatorname{ALK}(\mathrm{C} 1156 \mathrm{Y})$ & 100 & 89 & 84 \\
\hline ALK(L1196M) & 80 & 79 & 86 \\
\hline AMPK-alpha1 & 79 & 91 & 100 \\
\hline AMPK-alpha2 & 83 & 100 & 100 \\
\hline ANKK1 & 80 & 92 & 92 \\
\hline ARK5 & 86 & 94 & 100 \\
\hline ASK1 & 74 & 88 & 100 \\
\hline ASK2 & 100 & 100 & 100 \\
\hline AURKA & 92 & 78 & 100 \\
\hline AURKB & 100 & 99 & 100 \\
\hline AURKC & 95 & 100 & 100 \\
\hline AXL & 100 & 97 & 100 \\
\hline BIKE & 73 & 100 & 100 \\
\hline BLK & 55 & 95 & 100 \\
\hline BMPR1A & 81 & 97 & 100 \\
\hline BMPR1B & 88 & 94 & 99 \\
\hline BMPR2 & 100 & 100 & 100 \\
\hline BMX & 76 & 90 & 100 \\
\hline BRAF & 86 & 95 & 99 \\
\hline BRAF(V600E) & 100 & 97 & 95 \\
\hline BRK & 66 & 100 & 100 \\
\hline BRSK1 & 69 & 100 & 100 \\
\hline BRSK2 & 78 & 91 & 100 \\
\hline BTK & 100 & 100 & 100 \\
\hline BUB1 & 100 & 100 & 100 \\
\hline CAMK1 & 100 & 92 & 100 \\
\hline CAMK1B & 95 & 92 & 95 \\
\hline CAMK1D & 98 & 86 & 100 \\
\hline CAMK1G & 74 & 95 & 100 \\
\hline
\end{tabular}




\begin{tabular}{|c|c|c|c|}
\hline CAMK2A & 76 & 100 & 100 \\
\hline CAMK2B & 81 & 93 & 100 \\
\hline CAMK2D & 82 & 92 & 100 \\
\hline CAMK2G & 79 & 97 & 100 \\
\hline CAMK4 & 98 & 100 & 100 \\
\hline CAMKK1 & 84 & 100 & 100 \\
\hline CAMKK2 & 80 & 88 & 100 \\
\hline CASK & 83 & 100 & 100 \\
\hline CDC2L1 & 74 & 100 & 100 \\
\hline CDC2L2 & 87 & 95 & 100 \\
\hline CDC2L5 & 100 & 100 & 98 \\
\hline CDK11 & 71 & 90 & 100 \\
\hline CDK2 & 81 & 100 & 100 \\
\hline CDK3 & 87 & 100 & 100 \\
\hline CDK4 & 100 & 100 & 93 \\
\hline CDK4-cyclinD1 & 100 & 90 & 100 \\
\hline CDK4-cyclinD3 & 95 & 100 & 100 \\
\hline CDK5 & 71 & 98 & 100 \\
\hline CDK7 & 94 & 100 & 100 \\
\hline CDK8 & 78 & 100 & 100 \\
\hline CDK9 & 74 & 98 & 100 \\
\hline CDKL1 & 72 & 80 & 100 \\
\hline CDKL2 & 75 & 95 & 100 \\
\hline CDKL3 & 75 & 100 & 98 \\
\hline CDKL5 & 97 & 100 & 100 \\
\hline CHEK1 & 79 & 59 & 100 \\
\hline CHEK2 & 72 & 100 & 100 \\
\hline CIT & 73 & 98 & 100 \\
\hline CLK1 & 90 & 100 & 100 \\
\hline CLK2 & 72 & 100 & 94 \\
\hline CLK3 & 97 & 97 & 100 \\
\hline CLK4 & 76 & 85 & 98 \\
\hline CSF1R & 36 & 80 & 99 \\
\hline CSF1R-autoinhibited & 90 & 100 & 100 \\
\hline CSK & 85 & 100 & 100 \\
\hline CSNK1A1 & 62 & 97 & 100 \\
\hline CSNK1A1L & 78 & 100 & 100 \\
\hline CSNK1D & 54 & 87 & 95 \\
\hline CSNK1E & 82 & 100 & 100 \\
\hline CSNK1G1 & 86 & 97 & 100 \\
\hline CSNK1G2 & 81 & 80 & 100 \\
\hline CSNK1G3 & 87 & 100 & 100 \\
\hline CSNK2A1 & 93 & 95 & 92 \\
\hline
\end{tabular}




\begin{tabular}{|c|c|c|c|}
\hline CSNK2A2 & 87 & 93 & 100 \\
\hline CTK & 98 & 96 & 83 \\
\hline DAPK1 & 72 & 84 & 100 \\
\hline DAPK2 & 60 & 83 & 100 \\
\hline DAPK3 & 62 & 97 & 100 \\
\hline DCAMKL1 & 91 & 7.9 & 48 \\
\hline DCAMKL2 & 87 & 3 & 52 \\
\hline DCAMKL3 & 81 & 86 & 100 \\
\hline DDR1 & 89 & 100 & 100 \\
\hline DDR2 & 100 & 100 & 100 \\
\hline DLK & 90 & 86 & 87 \\
\hline DMPK & 99 & 100 & 100 \\
\hline DMPK2 & 91 & 93 & 100 \\
\hline DRAK1 & 95 & 100 & 100 \\
\hline DRAK2 & 100 & 100 & 100 \\
\hline DYRK1A & 100 & 87 & 96 \\
\hline DYRK1B & 69 & 87 & 87 \\
\hline DYRK2 & 100 & 100 & 100 \\
\hline EGFR & 90 & 100 & 100 \\
\hline EGFR(E746-A750del) & 67 & 79 & 100 \\
\hline EGFR(G719C) & 34 & 100 & 100 \\
\hline EGFR(G719S) & 48 & 100 & 100 \\
\hline EGFR(L747-E749del, A750P) & 95 & 97 & 98 \\
\hline EGFR(L747-S752del, P753S) & 99 & 91 & 100 \\
\hline EGFR(L747-T751del,Sins) & 52 & 100 & 100 \\
\hline EGFR(L858R) & 79 & 100 & 100 \\
\hline EGFR(L858R,T790M) & 100 & 80 & 100 \\
\hline EGFR(L861Q) & 38 & 100 & 100 \\
\hline EGFR(S752-I759del) & 47 & 100 & 100 \\
\hline EGFR(T790M) & 100 & 93 & 100 \\
\hline EIF2AK1 & 73 & 100 & 100 \\
\hline EPHA1 & 54 & 100 & 100 \\
\hline EPHA2 & 91 & 91 & 100 \\
\hline EPHA3 & 99 & 100 & 100 \\
\hline EPHA4 & 87 & 68 & 100 \\
\hline EPHA5 & 65 & 95 & 100 \\
\hline EPHA6 & 83 & 86 & 100 \\
\hline EPHA7 & 71 & 97 & 100 \\
\hline EPHA8 & 64 & 100 & 100 \\
\hline EPHB1 & 48 & 93 & 100 \\
\hline EPHB2 & 53 & 99 & 100 \\
\hline EPHB3 & 70 & 100 & 100 \\
\hline EPHB4 & 61 & 100 & 100 \\
\hline
\end{tabular}




\begin{tabular}{|c|c|c|c|}
\hline EPHB6 & 45 & 100 & 100 \\
\hline ERBB2 & 98 & 100 & 93 \\
\hline ERBB3 & 100 & 100 & 100 \\
\hline ERBB4 & 57 & 100 & 100 \\
\hline ERK1 & 88 & 92 & 100 \\
\hline ERK2 & 60 & 98 & 100 \\
\hline ERK3 & 89 & 100 & 100 \\
\hline ERK4 & 84 & 100 & 100 \\
\hline ERK5 & 80 & 30 & 100 \\
\hline ERK8 & 86 & 100 & 100 \\
\hline ERN1 & 93 & 88 & 100 \\
\hline FAK & 0.55 & 0.1 & 0.45 \\
\hline FER & 74 & 57 & 100 \\
\hline FES & 81 & 97 & 100 \\
\hline FGFR1 & 75 & 90 & 100 \\
\hline FGFR2 & 80 & 100 & 100 \\
\hline FGFR3 & 92 & 98 & 100 \\
\hline FGFR3(G697C) & 78 & 100 & 100 \\
\hline FGFR4 & 90 & 88 & 100 \\
\hline FGR & 77 & 100 & 100 \\
\hline FLT1 & 87 & 100 & 100 \\
\hline FLT3 & 100 & 99 & 100 \\
\hline FLT3(D835H) & 67 & 100 & 100 \\
\hline FLT3(D835V) & 57 & 97 & 100 \\
\hline FLT3(D835Y) & 76 & 91 & 100 \\
\hline FLT3(ITD) & 83 & 100 & 100 \\
\hline FLT3(ITD,D835V) & 100 & 100 & 100 \\
\hline FLT3(ITD,F691L) & 100 & 93 & 100 \\
\hline FLT3(K663Q) & 96 & 96 & 98 \\
\hline FLT3(N841I) & 98 & 83 & 100 \\
\hline FLT3(R834Q) & 100 & 81 & 99 \\
\hline FLT3-autoinhibited & 100 & 99 & 100 \\
\hline FLT4 & 92 & 100 & 100 \\
\hline FRK & 37 & 99 & 100 \\
\hline FYN & 73 & 93 & 100 \\
\hline GAK & 4.2 & 31 & 98 \\
\hline GCN2(Kin.Dom.2,S808G) & 88 & 94 & 100 \\
\hline GRK1 & 86 & 81 & 100 \\
\hline GRK2 & 100 & 100 & 93 \\
\hline GRK3 & 100 & 81 & 92 \\
\hline GRK4 & 72 & 97 & 100 \\
\hline GRK7 & 97 & 100 & 100 \\
\hline GSK3A & 82 & 100 & 100 \\
\hline
\end{tabular}




\begin{tabular}{|c|c|c|c|}
\hline GSK3B & 95 & 99 & 96 \\
\hline HASPIN & 96 & 89 & 100 \\
\hline HCK & 69 & 95 & 96 \\
\hline HIPK1 & 83 & 94 & 100 \\
\hline HIPK2 & 100 & 100 & 92 \\
\hline HIPK3 & 100 & 100 & 100 \\
\hline HIPK4 & 82 & 100 & 91 \\
\hline HPK1 & 68 & 100 & 91 \\
\hline HUNK & 95 & 97 & 100 \\
\hline ICK & 100 & 100 & 93 \\
\hline IGF1R & 86 & 40 & 100 \\
\hline IKK-alpha & 100 & 100 & 100 \\
\hline IKK-beta & 100 & 96 & 100 \\
\hline IKK-epsilon & 78 & 100 & 100 \\
\hline INSR & 83 & 40 & 81 \\
\hline INSRR & 84 & 22 & 88 \\
\hline IRAK1 & 100 & 100 & 100 \\
\hline IRAK3 & 80 & 99 & 100 \\
\hline IRAK4 & 100 & 88 & 100 \\
\hline ITK & 87 & 99 & 100 \\
\hline JAK1(JH1domain-catalytic) & 85 & 100 & 100 \\
\hline JAK1(JH2domain-pseudokinase) & 85 & 89 & 92 \\
\hline JAK2(JH1domain-catalytic) & 100 & 99 & 93 \\
\hline JAK3(JH1domain-catalytic) & 83 & 100 & 92 \\
\hline JNK1 & 100 & 100 & 94 \\
\hline JNK2 & 100 & 98 & 100 \\
\hline JNK3 & 100 & 97 & 100 \\
\hline KIT & 54 & 100 & 100 \\
\hline KIT(A829P) & 47 & 100 & 100 \\
\hline KIT(D816H) & 22 & 100 & 100 \\
\hline KIT(D816V) & 11 & 100 & 100 \\
\hline KIT(L576P) & 47 & 100 & 100 \\
\hline KIT(V559D) & 56 & 100 & 100 \\
\hline KIT(V559D,T670I) & 86 & 95 & 100 \\
\hline KIT(V559D,V654A) & 100 & 100 & 100 \\
\hline KIT-autoinhibited & 100 & 100 & 91 \\
\hline LATS1 & 77 & 50 & 100 \\
\hline LATS2 & 100 & 98 & 100 \\
\hline LCK & 45 & 100 & 100 \\
\hline LIMK1 & 96 & 95 & 100 \\
\hline LIMK2 & 91 & 98 & 100 \\
\hline LKB1 & 44 & 100 & 100 \\
\hline LOK & 84 & 99 & 100 \\
\hline
\end{tabular}




\begin{tabular}{|c|c|c|c|}
\hline LRRK2 & 98 & 32 & 70 \\
\hline LRRK2(G2019S) & 100 & 21 & 67 \\
\hline LTK & 81 & 97 & 100 \\
\hline LYN & 69 & 100 & 100 \\
\hline LZK & 100 & 100 & 100 \\
\hline MAK & 71 & 100 & 100 \\
\hline MAP3K1 & 100 & 100 & 100 \\
\hline MAP3K15 & 90 & 84 & 95 \\
\hline MAP3K2 & 100 & 100 & 97 \\
\hline MAP3K3 & 100 & 100 & 100 \\
\hline MAP3K4 & 73 & 100 & 100 \\
\hline MAP4K2 & 100 & 94 & 90 \\
\hline MAP4K3 & 100 & 100 & 100 \\
\hline MAP4K4 & 79 & 100 & 100 \\
\hline MAP4K5 & 82 & 91 & 100 \\
\hline MAPKAPK2 & 100 & 100 & 100 \\
\hline MAPKAPK5 & 100 & 93 & 100 \\
\hline MARK1 & 87 & 89 & 99 \\
\hline MARK2 & 100 & 100 & 100 \\
\hline MARK3 & 96 & 81 & 100 \\
\hline MARK4 & 83 & 87 & 100 \\
\hline MAST1 & 84 & 100 & 81 \\
\hline MEK1 & 100 & 95 & 96 \\
\hline MEK2 & 100 & 99 & 97 \\
\hline MEK3 & 92 & 100 & 98 \\
\hline MEK4 & 100 & 100 & 100 \\
\hline MEK5 & 22 & 100 & 100 \\
\hline MEK6 & 100 & 98 & 100 \\
\hline MELK & 99 & 88 & 100 \\
\hline MERTK & 93 & 100 & 100 \\
\hline MET & 92 & 97 & 100 \\
\hline MET(M1250T) & 82 & 100 & 100 \\
\hline MET(Y1235D) & 90 & 98 & 100 \\
\hline MINK & 100 & 100 & 84 \\
\hline MKK7 & 100 & 100 & 100 \\
\hline MKNK1 & 100 & 96 & 100 \\
\hline MKNK2 & 80 & 90 & 100 \\
\hline MLCK & 94 & 100 & 100 \\
\hline MLK1 & 94 & 41 & 100 \\
\hline MLK2 & 96 & 76 & 100 \\
\hline MLK3 & 73 & 100 & 100 \\
\hline MRCKA & 98 & 84 & 100 \\
\hline MRCKB & 100 & 100 & 100 \\
\hline
\end{tabular}




\begin{tabular}{|c|c|c|c|}
\hline MST1 & 89 & 100 & 96 \\
\hline MST1R & 90 & 100 & 100 \\
\hline MST2 & 100 & 100 & 100 \\
\hline MST3 & 89 & 90 & 100 \\
\hline MST4 & 100 & 100 & 93 \\
\hline MTOR & 85 & 31 & 94 \\
\hline MUSK & 92 & 100 & 100 \\
\hline MYLK & 53 & 36 & 96 \\
\hline MYLK2 & 84 & 97 & 100 \\
\hline MYLK4 & 77 & 100 & 100 \\
\hline MYO3A & 79 & 100 & 100 \\
\hline MYO3B & 74 & 100 & 100 \\
\hline NDR1 & 97 & 98 & 92 \\
\hline NDR2 & 86 & 64 & 100 \\
\hline NEK1 & 78 & 97 & 100 \\
\hline NEK10 & 100 & 94 & 99 \\
\hline NEK11 & 100 & 100 & 100 \\
\hline NEK2 & 84 & 100 & 100 \\
\hline NEK3 & 88 & 100 & 89 \\
\hline NEK4 & 100 & 100 & 100 \\
\hline NEK5 & 97 & 100 & 100 \\
\hline NEK6 & 78 & 90 & 100 \\
\hline NEK7 & 63 & 100 & 100 \\
\hline NEK9 & 84 & 100 & 100 \\
\hline NIK & 85 & 93 & 98 \\
\hline NIM1 & 100 & 100 & 100 \\
\hline NLK & 59 & 93 & 100 \\
\hline OSR1 & 100 & 100 & 100 \\
\hline p38-alpha & 75 & 93 & 96 \\
\hline p38-beta & 55 & 95 & 100 \\
\hline p38-delta & 82 & 100 & 100 \\
\hline p38-gamma & 67 & 95 & 100 \\
\hline PAK1 & 64 & 92 & 100 \\
\hline PAK2 & 72 & 89 & 99 \\
\hline PAK3 & 78 & 92 & 100 \\
\hline PAK4 & 65 & 100 & 100 \\
\hline PAK6 & 97 & 100 & 100 \\
\hline PAK7 & 97 & 88 & 100 \\
\hline PCTK1 & 100 & 100 & 100 \\
\hline PCTK2 & 76 & 100 & 100 \\
\hline PCTK3 & 90 & 98 & 100 \\
\hline PDGFRA & 100 & 88 & 100 \\
\hline PDGFRB & 50 & 100 & 100 \\
\hline
\end{tabular}




\begin{tabular}{|c|c|c|c|}
\hline PDPK1 & 76 & 96 & 100 \\
\hline PFCDPK1(P.falciparum) & 100 & 99 & 97 \\
\hline PFPK5(P.falciparum) & 100 & 97 & 100 \\
\hline PFTAIRE2 & 86 & 100 & 100 \\
\hline PFTK1 & 90 & 100 & 100 \\
\hline PHKG1 & 78 & 84 & 100 \\
\hline PHKG2 & 69 & 38 & 100 \\
\hline PIK3C2B & 86 & 100 & 100 \\
\hline PIK3C2G & 69 & 100 & 100 \\
\hline PIK3CA & 100 & 100 & 100 \\
\hline PIK3CA(C420R) & 90 & 100 & 100 \\
\hline PIK3CA(E542K) & 100 & 93 & 95 \\
\hline PIK3CA(E545A) & 84 & 100 & 98 \\
\hline PIK3CA(E545K) & 90 & 100 & 92 \\
\hline PIK3CA(H1047L) & 66 & 80 & 84 \\
\hline PIK3CA(H1047Y) & 91 & 100 & 100 \\
\hline PIK3CA(I800L) & 100 & 70 & 94 \\
\hline PIK3CA(M1043I) & 87 & 100 & 100 \\
\hline PIK3CA(Q546K) & 100 & 94 & 94 \\
\hline PIK3CB & 97 & 87 & 88 \\
\hline PIK3CD & 98 & 92 & 81 \\
\hline PIK3CG & 93 & 88 & 87 \\
\hline PIK4CB & 85 & 100 & 94 \\
\hline PIKFYVE & 98 & 60 & 88 \\
\hline PIM1 & 70 & 97 & 100 \\
\hline PIM2 & 92 & 100 & 100 \\
\hline PIM3 & 70 & 100 & 100 \\
\hline PIP5K1A & 100 & 100 & 100 \\
\hline PIP5K1C & 99 & 95 & 100 \\
\hline PIP5K2B & 76 & 39 & 100 \\
\hline PIP5K2C & 86 & 75 & 93 \\
\hline PKAC-alpha & 72 & 100 & 100 \\
\hline PKAC-beta & 82 & 100 & 100 \\
\hline PKMYT1 & 90 & 95 & 100 \\
\hline PKN1 & 69 & 100 & 100 \\
\hline PKN2 & 100 & 100 & 65 \\
\hline PKNB(M.tuberculosis) & 91 & 98 & 100 \\
\hline PLK1 & 100 & 0.7 & 10 \\
\hline PLK2 & 100 & 62 & 100 \\
\hline PLK3 & 52 & 20 & 59 \\
\hline PLK4 & 81 & 19 & 80 \\
\hline PRKCD & 86 & 94 & 100 \\
\hline PRKCE & 100 & 100 & 91 \\
\hline
\end{tabular}




\begin{tabular}{|c|c|c|c|}
\hline PRKCH & 89 & 94 & 99 \\
\hline PRKCI & 73 & 97 & 100 \\
\hline PRKCQ & 89 & 88 & 100 \\
\hline PRKD1 & 78 & 43 & 84 \\
\hline PRKD2 & 72 & 46 & 100 \\
\hline PRKD3 & 75 & 34 & 99 \\
\hline PRKG1 & 93 & 100 & 100 \\
\hline PRKG2 & 73 & 100 & 100 \\
\hline PRKR & 100 & 65 & 100 \\
\hline PRKX & 96 & 99 & 100 \\
\hline PRP4 & 100 & 100 & 100 \\
\hline PYK2 & 41 & 85 & 100 \\
\hline QSK & 100 & 94 & 100 \\
\hline RAF1 & 85 & 95 & 92 \\
\hline RET & 99 & 100 & 100 \\
\hline RET(M918T) & 87 & 100 & 100 \\
\hline RET(V804L) & 95 & 93 & 100 \\
\hline RET(V804M) & 100 & 73 & 100 \\
\hline RIOK1 & 62 & 100 & 100 \\
\hline RIOK2 & 82 & 100 & 100 \\
\hline RIOK3 & 79 & 99 & 100 \\
\hline RIPK1 & 100 & 96 & 100 \\
\hline RIPK2 & 88 & 95 & 100 \\
\hline RIPK4 & 100 & 100 & 99 \\
\hline RIPK5 & 100 & 60 & 93 \\
\hline ROCK1 & 100 & 100 & 100 \\
\hline ROCK2 & 100 & 100 & 100 \\
\hline ROS1 & 74 & 75 & 100 \\
\hline RPS6KA4(Kin.Dom.1-N-terminal) & 100 & 100 & 100 \\
\hline RPS6KA4(Kin.Dom.2-C-terminal) & 100 & 30 & 90 \\
\hline RPS6KA5(Kin.Dom.1-N-terminal) & 70 & 91 & 92 \\
\hline RPS6KA5(Kin.Dom.2-C-terminal) & 72 & 63 & 100 \\
\hline RSK1(Kin.Dom.1-N-terminal) & 78 & 83 & 100 \\
\hline RSK1(Kin.Dom.2-C-terminal) & 81 & 55 & 100 \\
\hline RSK2(Kin.Dom.1-N-terminal) & 99 & 97 & 100 \\
\hline RSK2(Kin.Dom.2-C-terminal) & 100 & 99 & 100 \\
\hline RSK3(Kin.Dom.1-N-terminal) & 83 & 100 & 100 \\
\hline RSK3(Kin.Dom.2-C-terminal) & 84 & 31 & 100 \\
\hline RSK4(Kin.Dom.1-N-terminal) & 100 & 91 & 100 \\
\hline RSK4(Kin.Dom.2-C-terminal) & 69 & 67 & 100 \\
\hline S6K1 & 100 & 100 & 100 \\
\hline SBK1 & 100 & 98 & 100 \\
\hline SGK & 100 & 100 & 99 \\
\hline
\end{tabular}




\begin{tabular}{|c|c|c|c|}
\hline SgK110 & 86 & 92 & 100 \\
\hline SGK2 & 99 & 98 & 92 \\
\hline SGK3 & 100 & 100 & 90 \\
\hline SIK & 69 & 100 & 100 \\
\hline SIK2 & 86 & 99 & 99 \\
\hline SLK & 70 & 100 & 100 \\
\hline SNARK & 100 & 91 & 86 \\
\hline SNRK & 94 & 100 & 100 \\
\hline SRC & 63 & 100 & 100 \\
\hline SRMS & 78 & 97 & 100 \\
\hline SRPK1 & 100 & 100 & 100 \\
\hline SRPK2 & 87 & 89 & 100 \\
\hline SRPK3 & 75 & 100 & 100 \\
\hline STK16 & 81 & 100 & 100 \\
\hline STK33 & 84 & 10 & 96 \\
\hline STK35 & 89 & 100 & 100 \\
\hline STK36 & 61 & 100 & 100 \\
\hline STK39 & 100 & 77 & 93 \\
\hline SYK & 77 & 89 & 100 \\
\hline TAK1 & 95 & 100 & 100 \\
\hline TAOK1 & 100 & 80 & 96 \\
\hline TAOK2 & 95 & 93 & 99 \\
\hline TAOK3 & 100 & 77 & 99 \\
\hline TBK1 & 71 & 88 & 100 \\
\hline TEC & 69 & 100 & 100 \\
\hline TESK1 & 81 & 100 & 100 \\
\hline TGFBR1 & 88 & 100 & 98 \\
\hline TGFBR2 & 96 & 98 & 100 \\
\hline TIE1 & 80 & 96 & 100 \\
\hline TIE2 & 83 & 100 & 100 \\
\hline TLK1 & 90 & 81 & 100 \\
\hline TLK2 & 100 & 100 & 100 \\
\hline TNIK & 94 & 99 & 100 \\
\hline TNK1 & 53 & 10 & 75 \\
\hline TNK2 & 20 & 87 & 100 \\
\hline TNNI3K & 79 & 88 & 100 \\
\hline TRKA & 100 & 100 & 94 \\
\hline TRKB & 100 & 100 & 100 \\
\hline TRKC & 100 & 100 & 100 \\
\hline TRPM6 & 100 & 92 & 100 \\
\hline TSSK1B & 74 & 68 & 98 \\
\hline TSSK3 & 98 & 98 & 79 \\
\hline TTK & 51 & 6.9 & 55 \\
\hline
\end{tabular}




\begin{tabular}{|l|l|l|l|} 
TXK & 73 & 99 & 100 \\
\hline TYK2(JH1domain-catalytic) & 84 & 97 & 100 \\
\hline TYK2(JH2domain-pseudokinase) & 100 & 100 & 100 \\
\hline TYRO3 & 92 & 88 & 100 \\
\hline ULK1 & 100 & 98 & 100 \\
\hline ULK2 & 100 & 81 & 98 \\
\hline ULK3 & 95 & 97 & 100 \\
\hline VEGFR2 & 100 & 95 & 99 \\
\hline VPS34 & 100 & 89 & 100 \\
\hline VRK2 & 91 & 97 & 100 \\
\hline WEE1 & 88 & 98 & 100 \\
\hline WEE2 & 65 & 100 & 100 \\
\hline WNK1 & 100 & 89 & 78 \\
\hline WNK2 & 74 & 100 & 98 \\
\hline WNK3 & 100 & 97 & 75 \\
\hline WNK4 & 100 & 100 & 100 \\
\hline YANK1 & 77 & 100 & 86 \\
\hline YANK2 & 85 & 100 & 98 \\
\hline YANK3 & 68 & 98 & 100 \\
\hline YES & 72 & 97 & 100 \\
\hline YSK1 & 79 & 100 & 100 \\
\hline YSK4 & 100 & 93 & 100 \\
\hline ZAK & 92 & 100 & 100 \\
\hline ZAP70 & 100 & 100 & 92 \\
\hline & & & \\
\hline
\end{tabular}


Supporting Table 2. Kinome Profiles of previously reported FAK inhibitors.
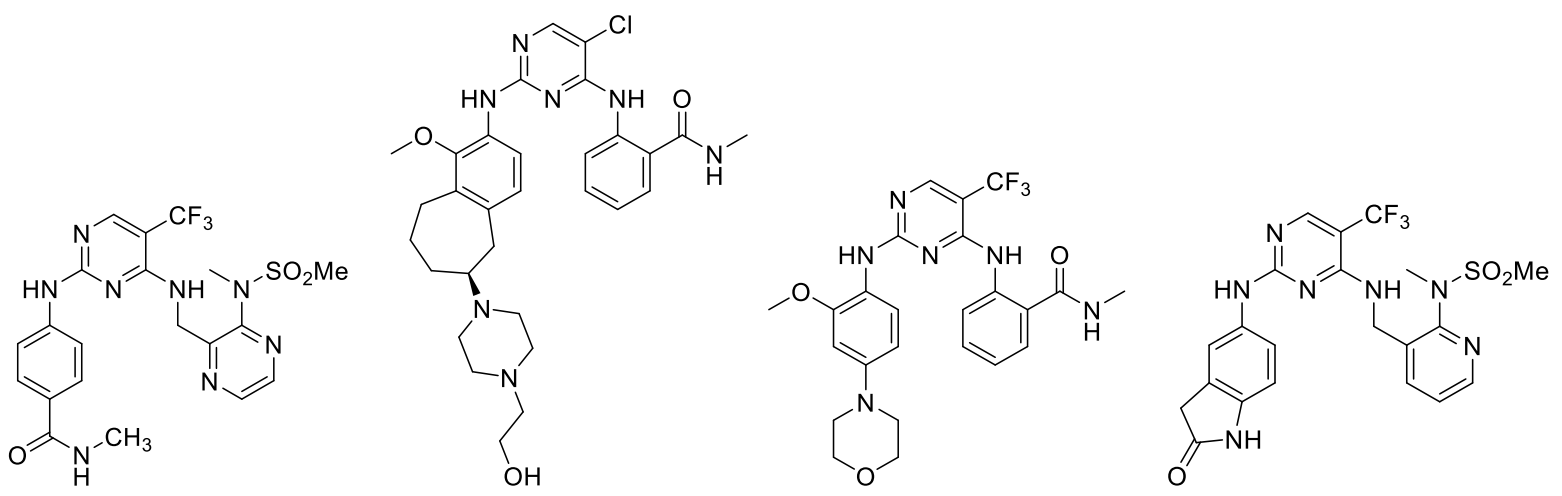

\begin{tabular}{|c|c|c|c|c|c|c|c|}
\hline \multicolumn{2}{|c|}{ Defactinib $^{1}$} & \multicolumn{2}{|c|}{ CEP-37440 ${ }^{2}$} & \multicolumn{2}{|c|}{ VS-4718 (PND-1186) ${ }^{3}$} & \multicolumn{2}{|l|}{ PF-562,271 } \\
\hline \multicolumn{2}{|c|}{$1 \mu \mathrm{M}$ concentration } & \multicolumn{2}{|c|}{$1 \mu \mathrm{M}$ concentration } & \multicolumn{2}{|c|}{$1 \mu \mathrm{M}$ concentration } & \multicolumn{2}{|l|}{--} \\
\hline Kinase & $\%$ Inhibition & Kinase & $\%$ Inhibition & Kinase & $\%$ Inhibition & Kinase & $\mathrm{IC}_{50}(\mathrm{nM})$ \\
\hline \multicolumn{2}{|c|}{39 kinases $>90 \%$ inhib. } & \multicolumn{2}{|c|}{37 kinases $>90 \%$ inhib. } & & & FAK & 1.5 \\
\hline FAK & 100 & FAK & 100 & FAK & 100 & PYK2 & 13 \\
\hline FLT3 & 99 & ALK & 97 & ACK1 & 84 & CDK1/cyclinB & 58 \\
\hline JAK1 & 100 & DYRK2 & 99 & AURKA & 92 & CDK2/cyclinE & 30 \\
\hline JAK2 & 100 & EGFR & 98 & FLT3 & 92 & CDK3/cyclinE & 47 \\
\hline JAK3 & 100 & RSK2 & 100 & LCK & 68 & CDK7/cyclinH/MAT1 & 97 \\
\hline STK16 & 99 & STK33 & 100 & TRKA & 73 & FLT3 & 97 \\
\hline TRKA & 98 & TNK1 & 100 & & & GSK3 $\beta$ & 133 \\
\hline
\end{tabular}

Full kinome profiles and/or biochemical IC50 values for off-targets can be found in the referenced publications. ${ }^{1-4}$ Detailed kinome profiling for PF-573228 (PF228) is unavailable. 
Supporting Figure 1. KINOMEscan of $1 @ 10 \mu \mathrm{M}$.

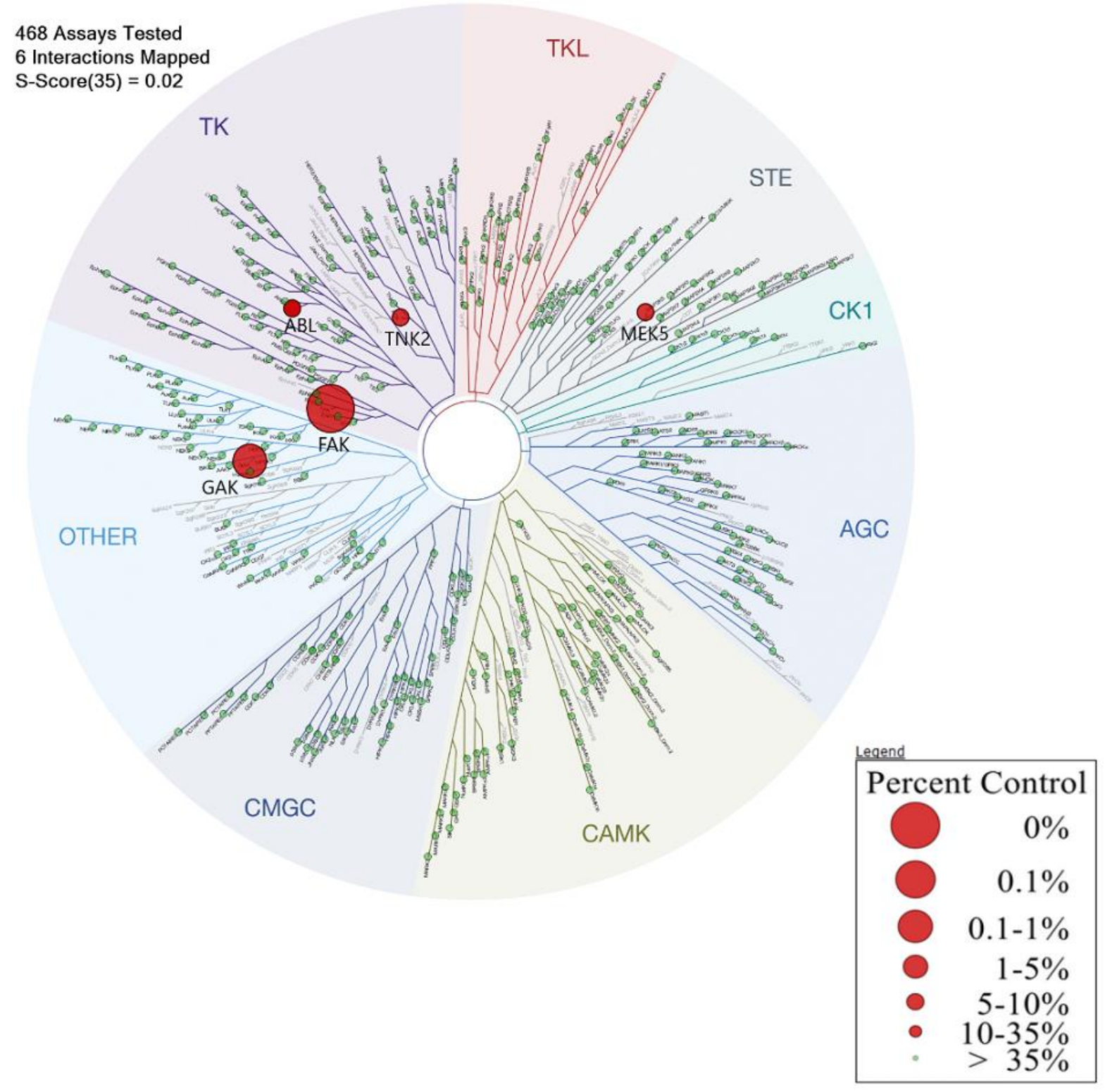


Supporting Figure 2. KINOMEscan of $4 @ 10 \mu \mathrm{M}$. All IC I $_{50}$ are reported as the average of at least two experiments.

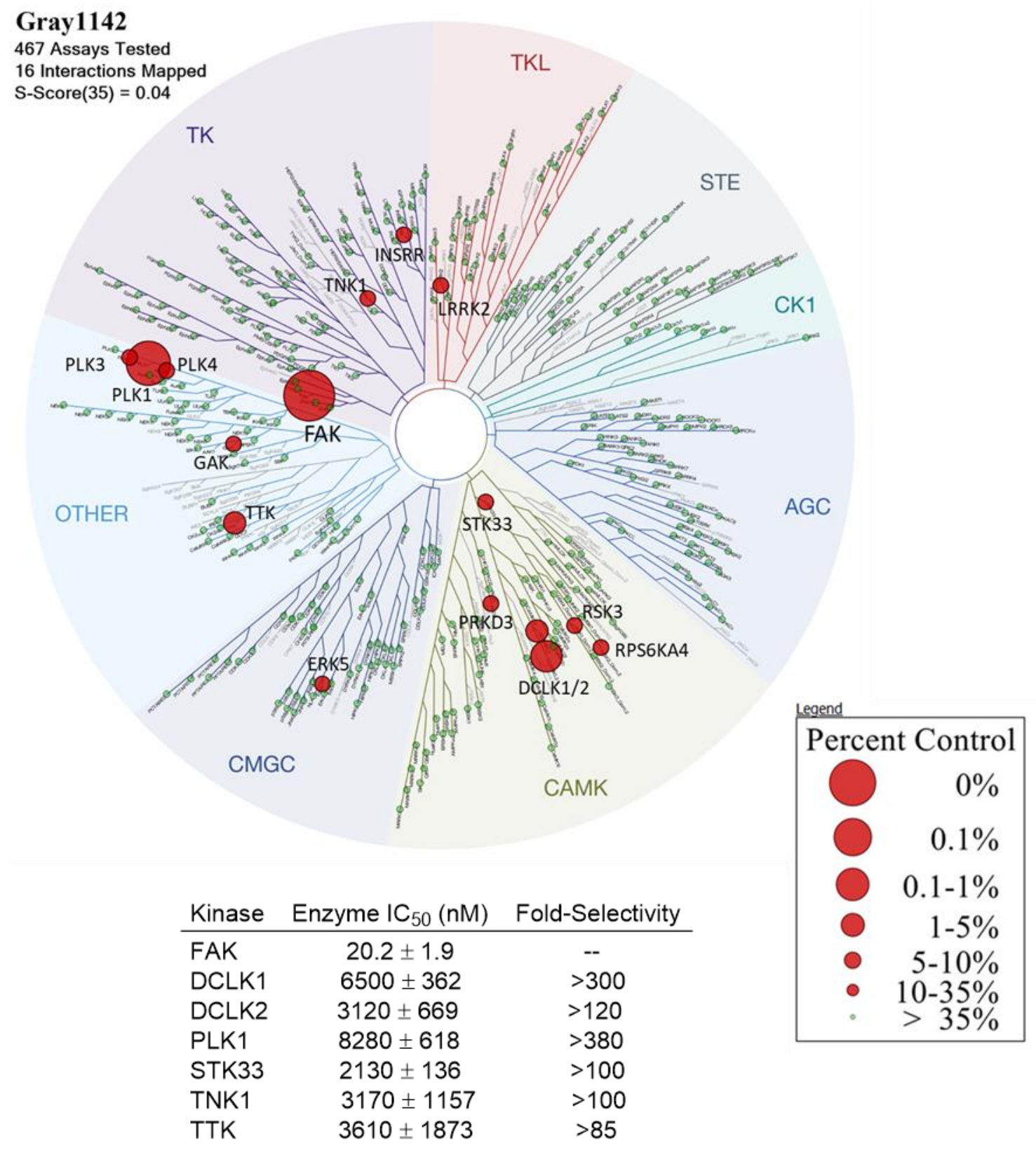


Supporting Figure 3. KINOMEscan of $4 @ 1 \mu \mathrm{M}$.

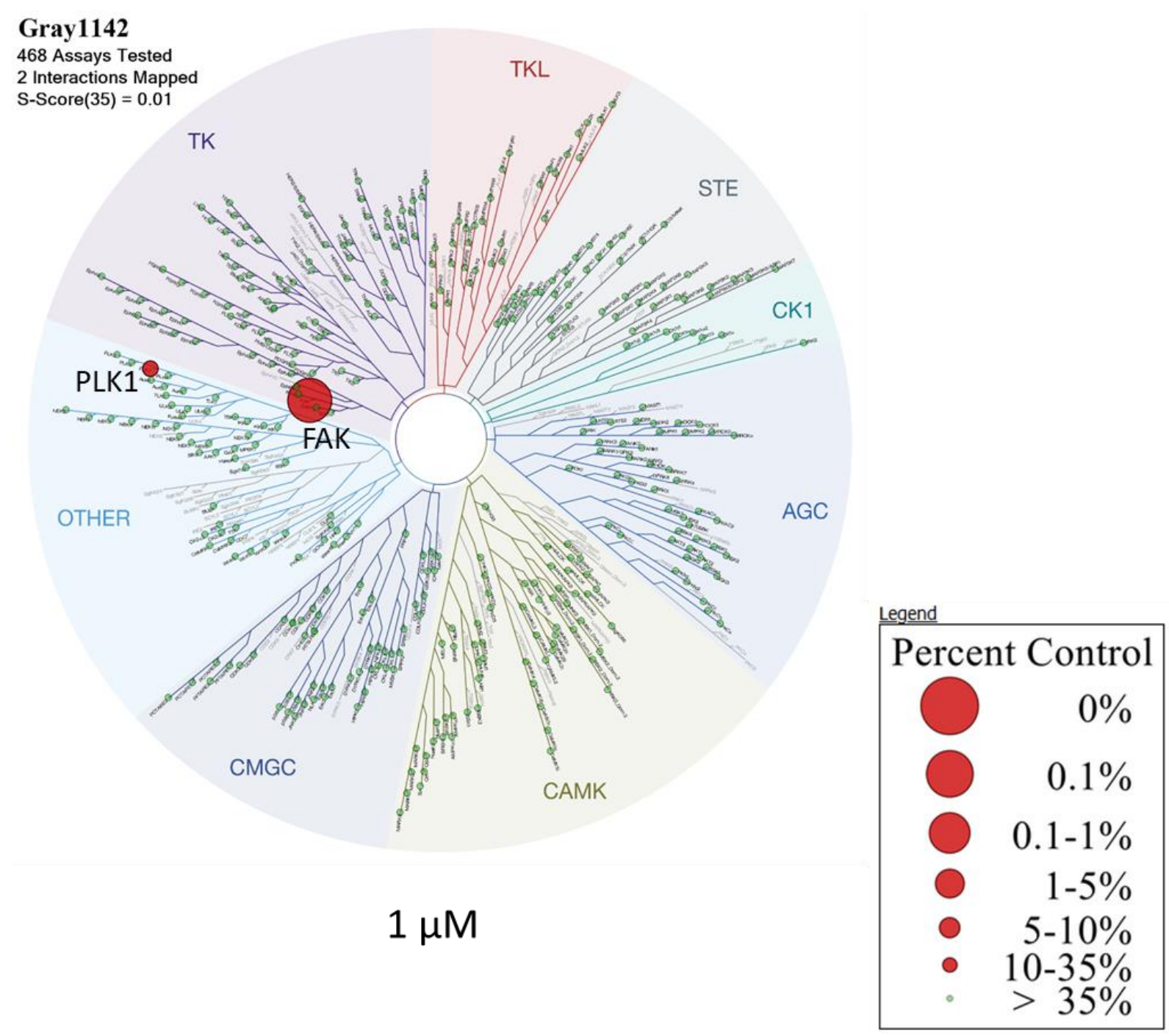




\section{Biochemical IC 50 Supporting Data}

Supporting Table 3. Biochemical IC $_{50}$ data of secondary amide series.

\begin{tabular}{|l|l|}
\hline \multicolumn{1}{|c|}{ Compound } & Biochemical $\mathrm{IC}_{50}(\mathrm{nM})$ \\
\hline $\mathbf{S}-46$ (BJG-01-177) & $228 \pm 3.8$ \\
\hline $\mathbf{S - 4 7}(\mathrm{BJG}-01-178)$ & $580 \pm 44.1$ \\
\hline $\mathbf{1}$ (BJG-01-181) & $62.2 \pm 2.1$ \\
\hline $\mathbf{S - 4 8}$ (BJG-02-015) & $90.4 \pm 5.2$ \\
\hline $\mathbf{S - 4 9}$ (BJG-03-106) & $48.7 \pm 1.8$ \\
\hline $\mathbf{2}$ (BJG-01-085) & $>10000$ \\
\hline $\mathbf{3}$ (BJG-02-063) & $132 \pm 3.7$ \\
\hline
\end{tabular}

Supporting Table 4. Biochemical IC50 data of $\mathrm{N}$-methyl amide series.

\begin{tabular}{|c|c|c|c|}
\hline Compound & Biochemical $\mathrm{IC}_{50}$ & Compound & Biochemical $\mathrm{IC}_{50}(\mathrm{nM})$ \\
\hline 4 (BJG-03-025) & $20.2 \pm 1.9$ & 23 (BJG-03-187-2) & $37.8 \pm 1.6$ \\
\hline 11 (BJG-03-161) & $30.3 \pm 0.5$ & 24 (BJG-04-015-2) & $73.9 \pm 1.7$ \\
\hline 13 (CRC-01-029) & $82.4 \pm 1.9$ & 25 (BJG-04-064-2) & $67.2 \pm 2.8$ \\
\hline 14 (BJG-03-181) & $46.9 \pm 2.6$ & 26 (BJG-03-204) & $196 \pm 11.5$ \\
\hline 15 (BJG-04-064-1) & $45.7 \pm 1.6$ & 27 (BJG-04-006-1) & $666 \pm 49.1$ \\
\hline 16 (BJG-03-187-1) & $31.5 \pm 0.8$ & 28 (BJG-04-005) & $77.7 \pm 4.0$ \\
\hline 17 (BJG-04-015-1) & $51.9 \pm 1.6$ & 12 (BJG-03-153-1) & $43.5 \pm 1.0$ \\
\hline 18 (BJG-03-179-1) & $54.5 \pm 1.3$ & $\mathbf{S - 5 0}$ (BJG-03-153-2) & $53.6 \pm 1.4$ \\
\hline 19 (BJG-03-179-2) & $465 \pm 31.9$ & S-51 (BJG-04-155) & $62.9 \pm 2.4$ \\
\hline 20 (BJG-03-184-1) & $173 \pm 8.6$ & S-52 (CRC-01-042) & $83.5 \pm 3.3$ \\
\hline 21 (BJG-03-184-2) & $45.4 \pm 2.1$ & S-53 (CRC-01-031) & $155 \pm 4.7$ \\
\hline 22 (BJG-04-013) & $670 \pm 12.7$ & & \\
\hline
\end{tabular}


Supporting Table 5. Biochemical IC $_{50}$ data of tricyclic core modifications.

\begin{tabular}{|l|l|}
\hline \multicolumn{1}{|c|}{ Compound } & \multicolumn{1}{c|}{ Biochemical $\mathrm{IC}_{50}(\mathrm{nM})$} \\
\hline $\mathbf{2 9}$ (BJG-03-084) & $510 \pm 34.9$ \\
\hline $\mathbf{3 0}$ (CRC-01-025) & $31.7 \pm 1.0$ \\
\hline $\mathbf{3 1}$ (CRC-01-033) & $58.8 \pm 1.0$ \\
\hline $\mathbf{3 2}$ (BJG-04-020) & $>10000$ \\
\hline $\mathbf{3 3}$ (BJG-04-035) & $134 \pm 1.2$ \\
\hline $\mathbf{3 4}$ (BJG-03-180) & $18.8 \pm 0.8$ \\
\hline $\mathbf{3 5}$ (BJG-03-201-1) & $22.9 \pm 0.4$ \\
\hline $\mathbf{3 6}$ (BJG-04-091) & $68.3 \pm 2.1$ \\
\hline $\mathbf{3 7}$ (BJG-04-067) & $46.6 \pm 1.3$ \\
\hline $\mathbf{3 8}$ (BJG-04-016) & $525 \pm 15.8$ \\
\hline
\end{tabular}




\section{Microsome Stability and PK Supporting Data}

Microsome stability and PK studies were conducted by the Michael Cameron lab, Scripps FL. PK studies were performed in C57BI/6 male mice, $\mathrm{n}=3$ for each route. BJG-03-025 was administered at a dose of $2 \mathrm{mg} / \mathrm{kg}$ (IV) or $10 \mathrm{mg} / \mathrm{kg}$ (PO) and formulated as a $0.2 \mathrm{mg} / \mathrm{mL}$ (IV) or $1 \mathrm{mg} / \mathrm{mL}$ (PO) aqueous solution containing 30\% captisol. Carbamazepine was used as internal standard.

Supporting Table 6. Compound stability and intrinsic clearance assessed in $1 \mathrm{mg} / \mathrm{mL}$ mouse hepatic microsomes.

\begin{tabular}{|l|r|r|}
\hline \multicolumn{1}{|c|}{ Compound ID } & $\mathrm{T}_{1 / 2}(\mathrm{~min})$ & $\begin{array}{c}\mathrm{CL}_{\text {int }} \\
(\mu \mathrm{L} / \mathrm{min} / \mathrm{mg})\end{array}$ \\
\hline $\mathbf{1}$ (BJG-01-181) & $>120$ & $<6$ \\
\hline $\mathbf{4}$ (BJG-03-025) & $>120$ & $<6$ \\
\hline $\mathbf{1 1}$ (BJG-03-161) & 28.2 & 25 \\
\hline $\mathbf{1 4}$ (BJG-03-181) & $>120$ & $<6$ \\
\hline $\mathbf{1 6}$ (BJG-03-187-1) & 55.7 & 12 \\
\hline $\mathbf{1 7}$ (BJG-04-015-1) & 51.0 & 14 \\
\hline $\mathbf{2 1}$ (BJG-03-184-2) & 113.8 & 6 \\
\hline $\mathbf{2 3}$ (BJG-03-187-2) & 25.5 & 27 \\
\hline $\mathbf{3 3}$ (BJG-04-035) & 64.1 & 11 \\
\hline
\end{tabular}


Supporting Table 7. PK data for 4 ( $2 \mathrm{mg} / \mathrm{kg}$ IV dose).

\begin{tabular}{|c|c|c|c|c|c|c|c|c|c|c|c|}
\hline Subject & $\mathbf{T}_{1 / 2}$ & $\mathbf{T}_{\max }$ & $\mathrm{C}_{\max }$ & $\mathrm{C}_{\max }$ & AUClast & AUClast & AUCINF_obs & AUC & Cl_obs & MRTINF_obs & Vss_ol \\
\hline Dose, $2 \mathrm{mg} / \mathrm{kg}$ & $h r$ & $h r$ & $n g / m L$ & $\mu M$ & $\min * n g / m L$ & $\mu M . h r$ & $\min * n g / m L$ & \%Extrap & $\mathrm{mL} / \mathrm{min} / \mathrm{kg}$ & $h r$ & $\mathrm{~L} / \mathrm{kg}$ \\
\hline IV Mouse-1 & 9.17 & 0.08 & 2480 & 4.40 & 132313 & 3.91 & 147310 & 10.18 & 13.58 & 7.21 & 5.88 \\
\hline IV Mouse-2 & 4.41 & 0.08 & 1570 & 2.79 & 78885 & 2.33 & 79916 & 1.29 & 25.03 & 2.97 & 4.46 \\
\hline IV Mouse-3 & 2.29 & 0.08 & 2770 & 4.92 & 113325 & 3.35 & 118208 & 4.13 & 16.92 & 1.85 & 1.88 \\
\hline Avg. & 5.29 & 0.08 & 2273 & 4.04 & 108174 & 3.20 & 115145 & 5.20 & 18.51 & 4.01 & 4.07 \\
\hline Std dev & 2.87 & & 511 & 0.91 & 22114 & 0.65 & 27599 & 3.71 & 4.81 & 2.31050712 & 1.66 \\
\hline
\end{tabular}

Supporting Table 8. PK data for 4 (10 mg/kg PO dose).

\begin{tabular}{|c|c|c|c|c|c|c|c|c|c|c|}
\hline Subject & $\mathbf{T}_{1 / 2}$ & $\mathbf{T}_{\max }$ & $\mathrm{C}_{\max }$ & $\mathrm{C}_{\max }$ & AUClast & AUClast & AUCINF_obs & $\mathbf{A U C}$ & Cl_obs & $\mathbf{F} \%$ \\
\hline Dose, $10 \mathrm{mg} / \mathrm{kg}$ & $h r$ & $h r$ & $n g / m L$ & $\mu M$ & $\min * n g / m L$ & $\mu M . h r$ & $\min * n g / m L$ & $\%$ Extrap & $\mathrm{mL} / \mathrm{min} / \mathrm{kg}$ & \\
\hline PO Mouse-4 & 4.88 & 4.00 & 249 & 0.44 & 148352 & 4.39 & 153928 & 3.62 & 64.97 & \\
\hline PO Mouse-5 & 2.58 & 4.00 & 213 & 0.38 & 70638 & 2.09 & 80122 & 11.84 & 124.81 & \\
\hline PO Mouse-6 & 2.34 & 4.00 & 254 & 0.45 & 78026 & 2.31 & 93695 & 16.72 & 106.73 & \\
\hline Avg. & 3.27 & 4.00 & 239 & 0.42 & 99005 & 2.93 & 109248 & 10.73 & 98.83 & 18.30 \\
\hline Std dev & 1.15 & & 18 & 0.03 & 35023 & 1.04 & 32075 & 5.41 & 25.06 & \\
\hline
\end{tabular}


Supporting Figure 4. Individual plots from PK study of 4 (IV dosing).
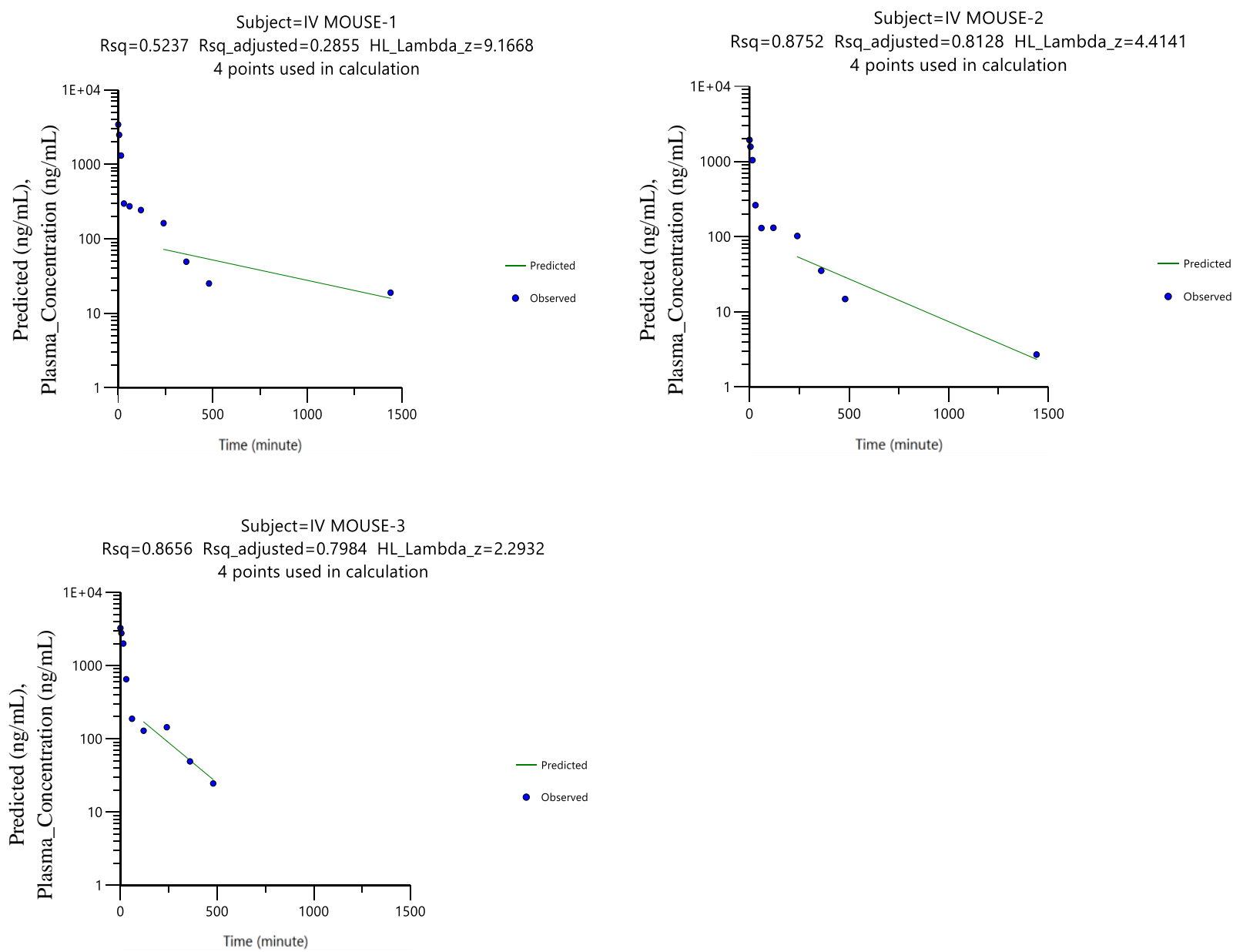
Supporting Figure 5. Individual plots from PK study of 4 (PO dosing).
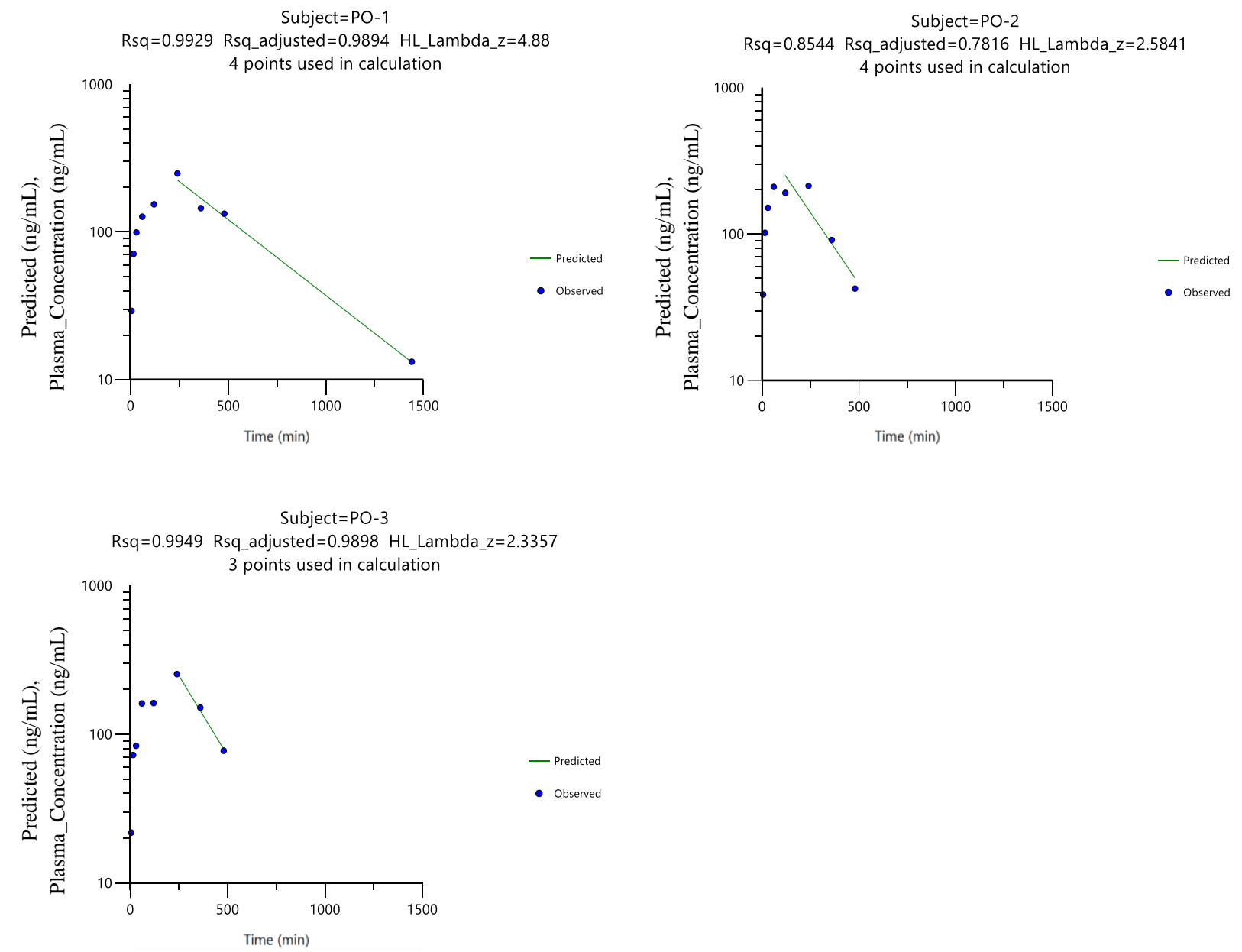


\title{
Kinetic Solubility, Thermodynamic Solubility, and Caco-2 Permeability Assay Data
}

Kinetic solubility, thermodynamic solubility, and Caco-2 permeability studies were conducted at BioDuro Shanghai. Kinetic solubility was determined by shaking sample stock in $\mathrm{pH}=7.4$ buffer at room temperature for 4 hours, followed by filtering and subjecting to LC-MS analysis. Atenolol was used as a reference. Thermodynamic solubility was determined by shaking sample stock in $\mathrm{pH}=7.4$ buffer at room temperature for 16 hours, followed by filtering and subjecting to LC-MS analysis. Caco-2 permeability assays were conducted by incubating both sides with sample stocks, comparing $\mathrm{t}=0 \mathrm{~min}$ and $\mathrm{t}=90 \mathrm{~min}$ samples by LC-MS. Propranolol, atenolol, and taxol were used as references. Permeability classifications are high $\left(\mathrm{P}_{\text {app }}>1010^{-6} \mathrm{~cm} / \mathrm{s}\right)$, medium $\left(\mathrm{P}_{\text {app }} 1-1010^{-6}\right.$ $\mathrm{cm} / \mathrm{s})$, or low $\left(\mathrm{P}_{\mathrm{app}}<110^{-6} \mathrm{~cm} / \mathrm{s}\right)$. Efflux ratios $>2.0$ suggest that the compound is a potential efflux transporter substrate.

\author{
Supporting Table 8. Kinetic solubility data for 4, 34, and 35 . \\ Compound \\ 4 (BJG-03-025) \\ 34 (BJG-03-180) \\ 35 (BJG-03-201-1) \\ Atenolol \\ Target concentration of test compound $=200 \mu \mathrm{M}$ \\ Solubility $(\mu \mathrm{M})$ in $\mathrm{pH}=7.4$ buffer \\ 167.0 \\ 186.5 \\ 128.0 \\ 474.5
}

\section{Supporting Table 9. Thermodynamic solubility data for 4, 34, and 35 .}

\section{Compound}

4 (BJG-03-025)

34 (BJG-03-180)

35 (BJG-03-201-1)
Solubility $(\mu \mathrm{M})$ in $\mathrm{pH}=7.4$ buffer

741.5

807.5

805.5

Theoretical solubility is $1000 \mu \mathrm{M}$. 
Supporting Table 10. Caco-2 permeability assay data for 4,34 , and 35 .

\begin{tabular}{|c|c|c|c|c|c|}
\hline \multirow[b]{2}{*}{ Compound } & \multicolumn{2}{|c|}{$P_{\text {app }}\left(10^{-6} \mathrm{~cm} / \mathrm{s}\right)$} & \multirow[b]{2}{*}{$\begin{array}{l}\text { Efflux } \\
\text { Ratio }\end{array}$} & \multicolumn{2}{|c|}{ Recovery (\%) } \\
\hline & $A \rightarrow B$ & $\mathbf{B} \rightarrow \mathbf{A}$ & & $A \rightarrow B$ & $\mathbf{B} \rightarrow \mathbf{A}$ \\
\hline 4 (BJG-03-025) & 5.8 & 23.7 & 4.1 & 108.7 & 117.4 \\
\hline 34 (BJG-03-180) & 8.3 & 18.5 & 2.2 & 114.8 & 103.6 \\
\hline 35 (BJG-03-201-1) & 7.6 & 22.1 & 2.9 & 105.7 & 106.7 \\
\hline Atenolol & 0.5 & 0.5 & 0.9 & 101.4 & 97.4 \\
\hline Propranolol & 40.7 & 30.8 & 0.8 & 103.3 & 92.9 \\
\hline Taxol & 0.1 & 3.3 & 27.0 & 119.8 & 132.3 \\
\hline
\end{tabular}

\section{Docking Analysis of Additional Compounds 3, 20, 26, 37, \& 38}

Supporting Figure 6. Compounds 3, 20, 26, 37, and 38 docked with FAK (PDB 6I8Z).

A
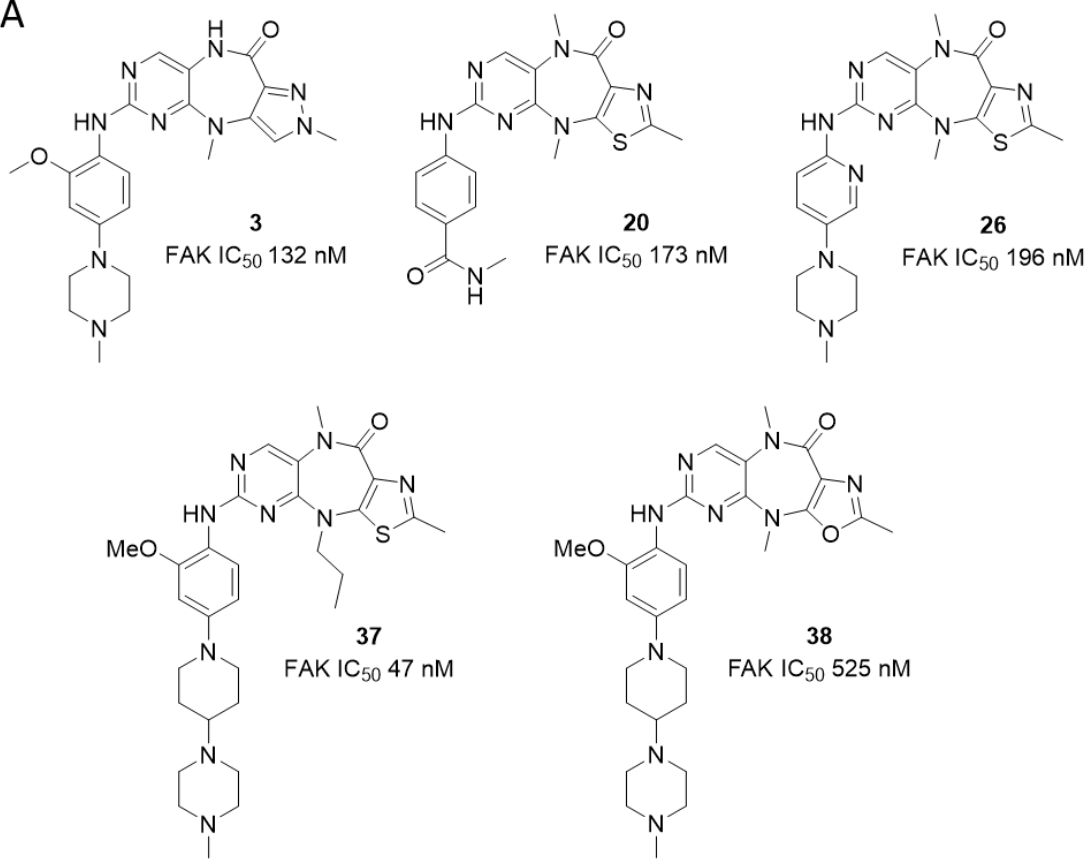
B
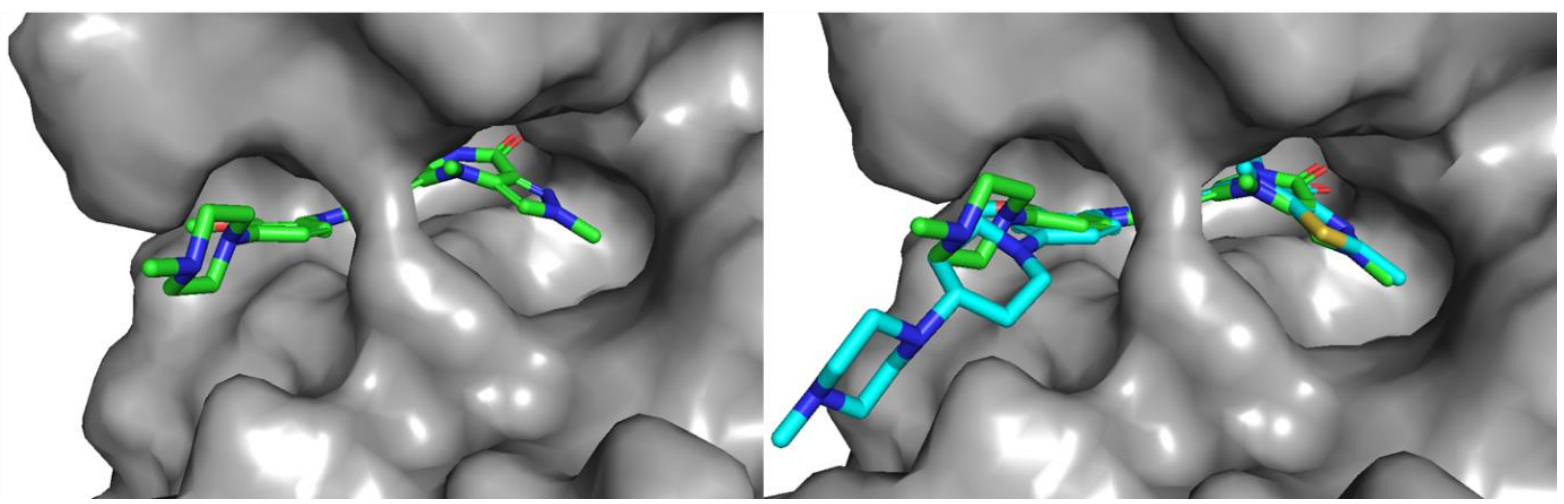

C
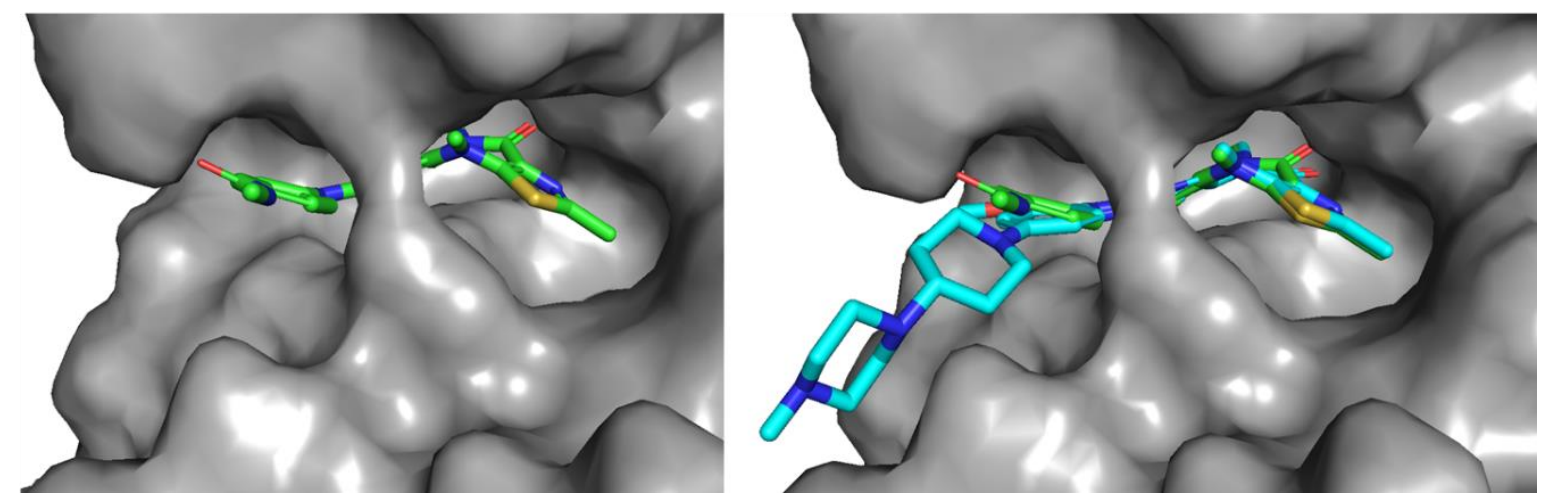

D
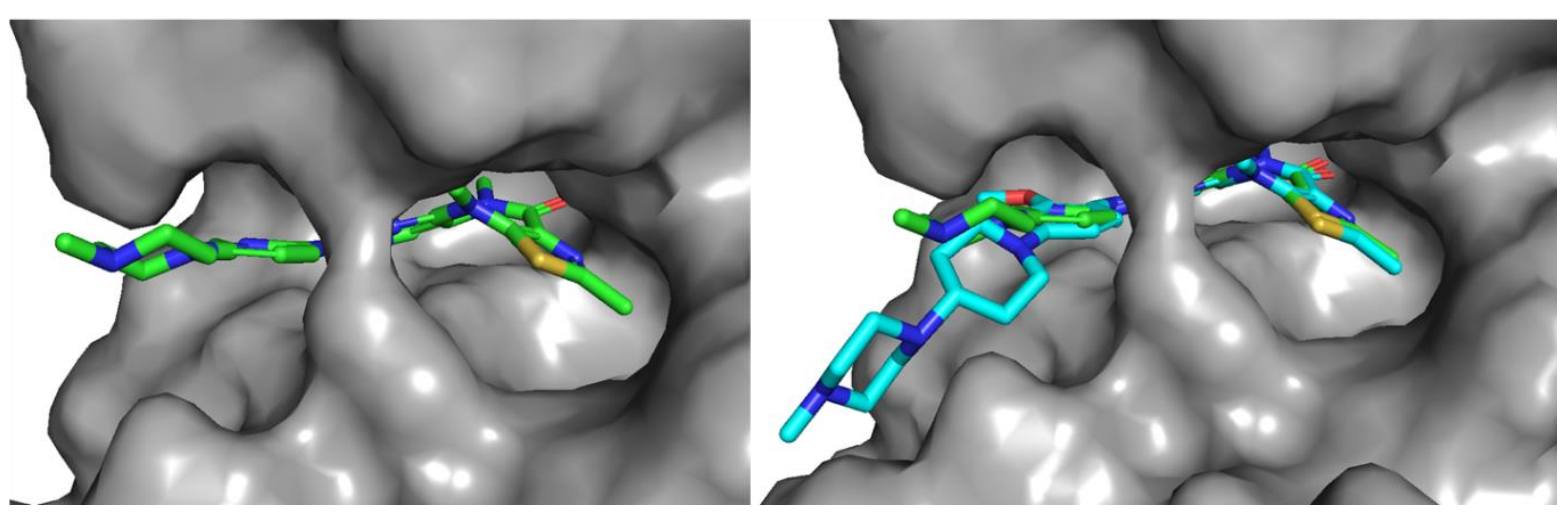

E

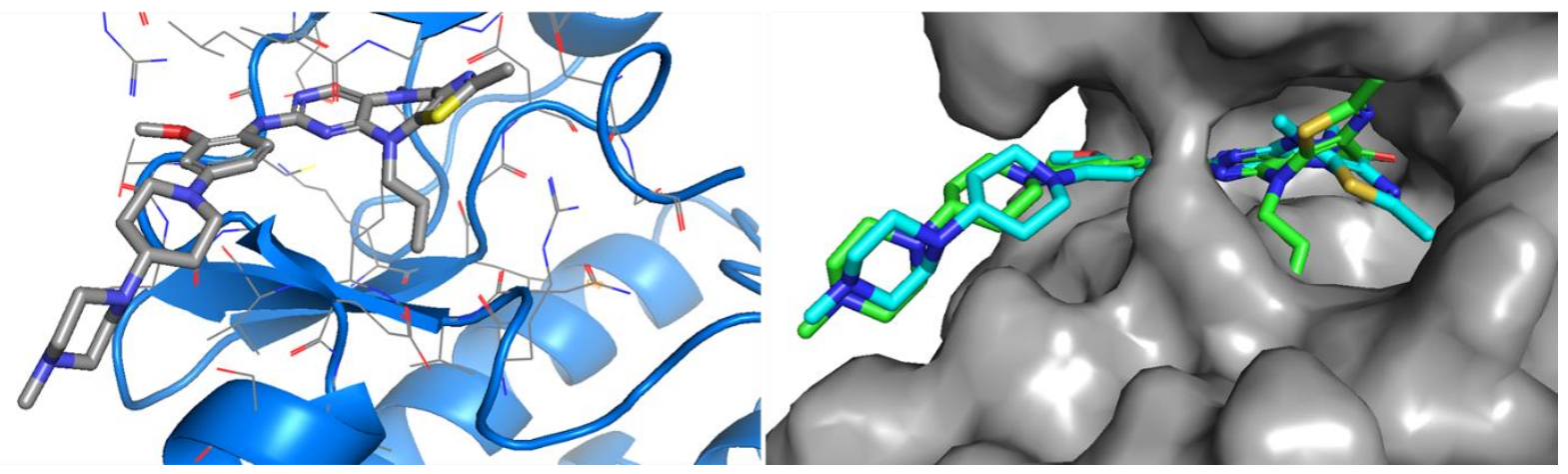




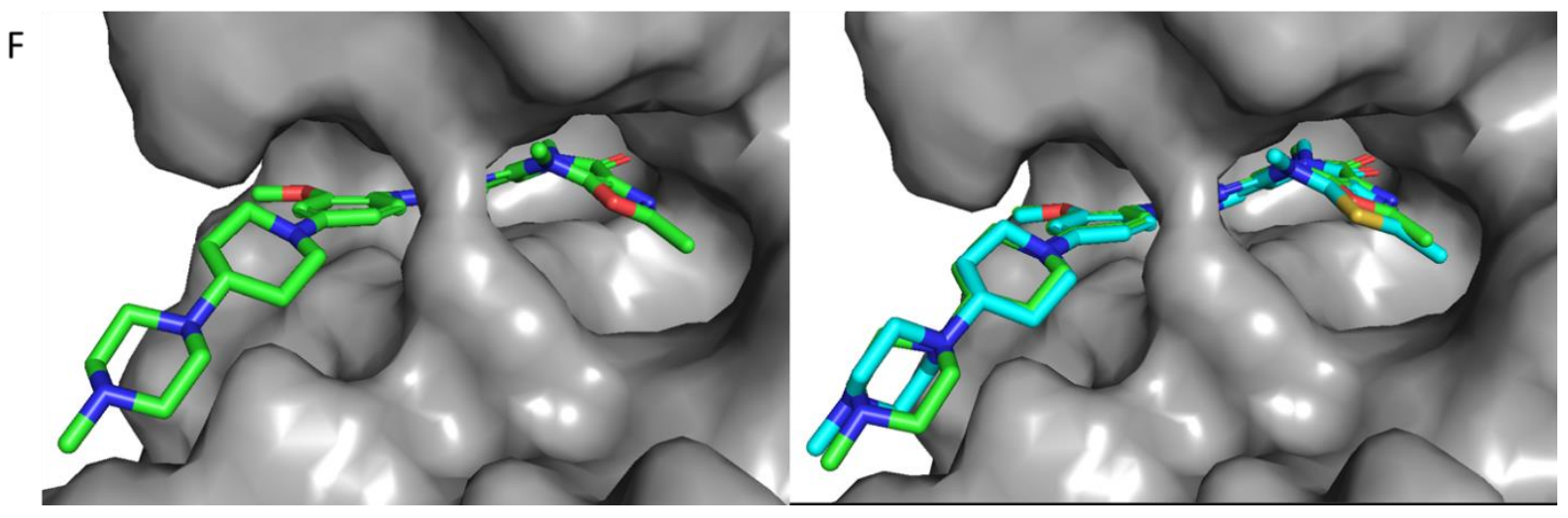

(A) Chemical structures of docked molecules. (B) Docking of $\mathbf{3}$ with FAK, and overlay with $\mathbf{4}$ (cyan). (C) Docking of 20 with FAK, and overlay with 4 (cyan). (D) Docking of 26 with FAK, and overlay with 4 (cyan). (E) Docking of 37 with FAK, and overlay with 4 (cyan). (F) Docking of $\mathbf{3 8}$ with FAK, and overlay with $\mathbf{4}$ (cyan). 


\section{General Chemistry Methods}

Analytical grade solvents and commercially available reagents were purchased from commercial sources and used directly without further purification unless otherwise stated. Thin-layer chromatography (TLC) was carried out on Merck $60 \mathrm{~F}_{254}$ precoated, glass silica plates which were visualized with either ultraviolet light or stained with $\mathrm{KMnO}_{4}$. Experiments were conducted under ambient conditions unless otherwise stated. ${ }^{1} \mathrm{H}-\mathrm{NMR},{ }^{13} \mathrm{C}-\mathrm{NMR}$, and ${ }^{19} \mathrm{~F}-\mathrm{NMR}$ spectra were recorded at room temperature using a Bruker $500\left({ }^{1} \mathrm{H}-\mathrm{NMR}\right.$ at $500 \mathrm{MHz},{ }^{13} \mathrm{C}-\mathrm{NMR}$ at $125 \mathrm{MHz}$, and ${ }^{19} \mathrm{~F}-\mathrm{NMR}$ at $471 \mathrm{MHz}$ ). Chemical shifts are reported in ppm with reference to solvent signals $\left[{ }^{1} \mathrm{H}-\mathrm{NMR}: \mathrm{CDCl}_{3}(7.26 \mathrm{ppm}), \mathrm{DMSO}-d_{6}(2.50 \mathrm{ppm}) ;{ }^{13} \mathrm{C}-\mathrm{NMR}: \mathrm{CDCl}_{3}(77.16 \mathrm{ppm}), \mathrm{DMSO}-d_{6}\right.$ (39.52 ppm)]. Signal patterns are indicated as s, singlet; br s, broad singlet; d, doublet; t, triplet, q, quartet; p, pentet; and m, multiplet. Mass spectrometry (MS) analysis was obtained on a Waters Acquity UPLC-MS system using electrospray ionization (ESI) in positive ion mode, reporting the molecular ion $[\mathrm{M}+\mathrm{H}]^{+},[\mathrm{M}+\mathrm{Na}]^{+}$, or a suitable fragment ion. Flash chromatography purification was conducted using an ISCO CombiFlash RF+ with RediSep Rf silica cartridges. Preparative reverse-phase HPLC purification was conducted using a Waters model 2545 pump and 2489 UV/Vis detector using SunFire Prep C18 $5 \mu \mathrm{m}$ columns $(18 \times 100 \mathrm{~mm}, 20 \mathrm{~mL} / \mathrm{min}$ flow rate; 30x250 mm, $40 \mathrm{~mL} / \mathrm{min}$ flow rate), and a gradient solvent system of water $(0.035 \%$ TFA)/methanol (0.035\% TFA) or water (0.035\% TFA)/acetonitrile $(0.035 \%$ TFA).

\footnotetext{
Abbreviations

$\mathrm{AcOH}$, glacial acetic acid; $\mathrm{AUC}_{\mathrm{inf}}$, area under the curve extrapolated to infinity; $\mathrm{BINAP},( \pm)-2,2^{\prime}-$ bis(diphenylphosphino)-1,1'-binaphthalene; CL, clearance; $C_{\max }$, maximum concentration; dba, dibenzylideneacetone; DCM, dichloromethane; DIPEA, diisopropylethylamine; DMF, N,Ndimethylformamide; DMSO, dimethyl sulfoxide; EtOAc, ethyl acetate; $F$, bioavailability; hex,
} 
hexanes; IV, intravenous; MeCN, acetonitrile; NBS, N-bromosuccinimide; PK, pharmacokinetics; PO, per os; $t_{1 / 2}$, half-life; SAR, structure-activity relationship; TFA, trifluoroacetic acid; THF, tetrahydrofuran; TLC, thin-layer chromatography; $T_{\max }$, time at $C_{\max }$; UPLC-MS, ultraperformance liquid chromatography-mass spectrometry; $V_{\mathrm{ss}}$, volume of distribution at steady state; XPhos, 2-dicyclohexylphosphino-2',4',6'-triisopropylbiphenyl.

\section{Experimental Procedures and Characterizations}

General Procedure A: Bromination of thiazoles with NBS. ${ }^{5}$

To a solution of thiazole-4-carboxylate $(1$ equiv) in acetonitrile $(0.3 \mathrm{M})$ was added $\mathrm{N}$ bromosuccinimide ( 2.5 equiv). The mixture was heated to $40{ }^{\circ} \mathrm{C}$ for 2 days. The reaction was then diluted with DCM and water. The organic layer was removed, and the aqueous layer extracted with DCM. The combined organic layers were washed twice with water, once with brine, dried over $\mathrm{MgSO}_{4}$, filtered and then concentrated. The brominated product was purified by ISCO flash chromatography (silica, EtOAc/hexanes).

General Procedure B: Alkylation and $\mathrm{S}_{\mathrm{N}}$ Ar reactions of aminothiazoles with $\mathrm{NaH}$ deprotonation.

A round-bottom flask was dried with a heat gun. Sodium hydride (1.3 equiv, 60\% dispersion in mineral oil) was added and washed twice with hexanes. Anhydrous THF (0.15 M) was added and the flask was cooled on an ice bath. To this suspension, a solution of aminothiazole (1.0 equiv, 0.2 $\mathrm{M}$ in THF) was added dropwise. The reaction was stirred for 30-60 minutes and then the desired electrophile (1.5 equiv) was added dropwise. The reaction was stirred overnight, slowly warming to room temperature. The reaction was quenched with water and extracted with EtOAc (4x50 mL). The combined organic layers were washed with brine, dried over $\mathrm{MgSO}_{4}$, filtered and concentrated. The product was purified by ISCO flash chromatography (silica, EtOAC/hexanes). 
$\underline{\text { General Procedure C: Formation of diazepinone via iron-mediated reductive lactamization. }}{ }^{6}$

A suspension of nitropyrimidine substrate (1.0 equiv) and iron powder (10 equiv) in glacial acetic acid $(0.07 \mathrm{M})$ was heated to $50{ }^{\circ} \mathrm{C}$ overnight. The reaction was cooled to room temperature and residual iron was removed with a magnetic wand. The crude reaction mixture was poured into a beaker with water and stirred at room temperature for 15-30 minutes. The resulting precipitate was collected by suction filtration, washing with water, and dried in vacuo to provide the tricyclic product, which was used without further purification.

General Procedure D: Alkylation of secondary amides. ${ }^{6}$

To a suspension of secondary amide (1.0 equiv) and alkyl iodide (2.0 equiv) in anhydrous DMF $(0.08 \mathrm{M})$ at $0{ }^{\circ} \mathrm{C}, \mathrm{NaH}$ (2.2 equiv, $60 \%$ dispersion in mineral oil) was added in a single portion. The reaction was typically stirred for 1-3 hours, or until UPLC-MS analysis showed complete consumption of starting material. The reaction was then quenched with water and extracted with DCM or EtOAc. The combined organic layers were washed twice with water, once with brine, dried over $\mathrm{MgSO}_{4}$, filtered and concentrated. If necessary, the product was purified by ISCO flash chromatography (silica, 0-10\% MeOH/DCM gradient).

General Procedure E: Pd-catalyzed amination of chloropyrimidines. ${ }^{6}$

To a 1-dram vial with a stir bar were added chloropyrimidine tricyclic core (1.0 equiv), $\operatorname{Pd}_{2}(\mathrm{dba})_{3}$ (0.10 equiv), XPhos ( 0.20 equiv), $\mathrm{K}_{2} \mathrm{CO}_{3}$ (3.0 equiv) and the desired aniline (1.30-1.50 equiv). Tert-butanol $(0.08 \mathrm{M})$ was added, the flask was sealed with a septum-lined cap, and the suspension was sparged with nitrogen for 10 minutes. The reaction was heated to $100{ }^{\circ} \mathrm{C}$ overnight, and then diluted with EtOAc $(10 \mathrm{~mL})$, filtered through Celite, concentrated in vacuo, and purified by reverse-phase preparative HPLC using a water $(0.035 \%$ TFA $) /$ methanol $(0.035 \%)$ or water $(0.035 \%$ TFA)/acetonitrile $(0.035 \%)$ gradient. 
General synthetic scheme for substituted anilines<smiles>[R]Oc1cc(F)ccc1[N+](=O)[O-]</smiles>

1.5-3.0 equiv<smiles>[R]Oc1cc(N2CCN([R])CC2)ccc1N</smiles>

$S_{N} A$ r reaction of para-nitroaryl fluorides and piperazines

A suspension of substituted para-nitroarylfluoride (1.0 equiv), substituted piperazine (1.5-3.0 equiv), and $\mathrm{K}_{2} \mathrm{CO}_{3}$ (2.0 equiv) in $\mathrm{DMF}(0.3-0.5 \mathrm{M})$ was heated to $100^{\circ} \mathrm{C}$ for 1 hour, or until UPLCMS analysis showed complete conversion of the aryl fluoride. The reaction was then cooled, diluted with ethyl acetate, washed three times with water, once with $1 \mathrm{M} \mathrm{HCl}$, and once with brine, dried over $\mathrm{MgSO} 4$, filtered, and concentrated. The crude $\mathrm{S}_{\mathrm{N}} \mathrm{Ar}$ product was generally $>95 \%$ pure by ${ }^{1} \mathrm{H}-\mathrm{NMR}$ analysis and was carried on to the reduction step without purification.

\section{Reduction of nitro group by catalytic hydrogenation}

A suspension of nitroaryl intermediate (1.0 equiv) and $\mathrm{Pd} / \mathrm{C}(0.10$ equiv, 10 wt \%) in methanol (0.1-0.2 M) was sparged with $\mathrm{H}_{2}$ for 10 minutes, and then stirred at room temperature under a balloon atmosphere of $\mathrm{H}_{2}$ until UPLC-MS analysis showed complete reduction of the nitro group. The reaction was then filtered through Celite, washing with additional $\mathrm{MeOH}$, and concentrated. The aniline product was used without further purification. 


\section{$\underline{\text { Synthetic Intermediates }}$}

Synthesis of 2-methylthiazole core
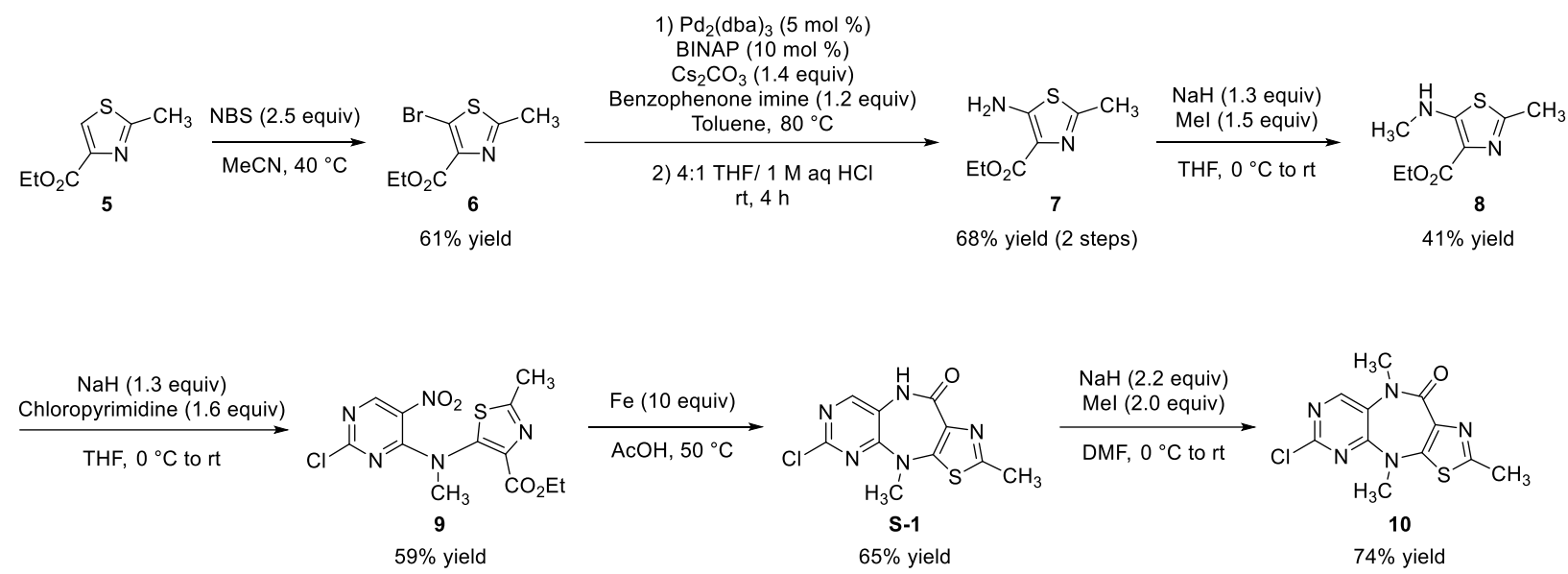

Note: While electron-rich bromothiazoles such as 6 require Pd-catalyzed amination with benzophenone imine followed by N-methylation, electron-deficient bromothiazoles (2trifluoromethyl, 2-H) and bromooxazoles successfully undergo $\mathrm{S}_{\mathrm{N}} \mathrm{Ar}$ reaction with methylamine.

\section{Ethyl 5-bromo-2-methylthiazole-4-carboxylate (6)}<smiles>CCOc1nc(C)sc1Br</smiles>

General Procedure A was followed on $14 \mathrm{mmol}$ scale using 2-methylthiazole-4-carboxylate 5 (2.417 g). The mixture was heated to $40{ }^{\circ} \mathrm{C}$ for 2 days. The reaction was then diluted with DCM $(100 \mathrm{~mL})$ and water $(200 \mathrm{~mL})$. The organic layer was removed, and the aqueous layer extracted with DCM $(3 \times 75 \mathrm{~mL})$. The combined organic layers were washed twice with water, once with brine, dried over $\mathrm{MgSO}_{4}$, filtered and then concentrated to provide a dark, viscous oil. ISCO flash chromatography (40 g silica, 20-80\% EtOAc/hexanes, $20 \mathrm{~min}$ gradient) provided the title compound as a light yellow solid ( $2.174 \mathrm{~g}, 61 \%$ yield).

${ }^{1} \mathrm{H}$ NMR $\left(500 \mathrm{MHz}, \mathrm{CDCl}_{3}\right) \delta 4.44(\mathrm{q}, J=7.2 \mathrm{~Hz}, 2 \mathrm{H}), 2.71(\mathrm{~s}, 3 \mathrm{H}), 1.42(\mathrm{t}, J=7.2 \mathrm{~Hz}, 3 \mathrm{H})$. LRMS (ESI) calculated for $\mathrm{C}_{7} \mathrm{H}_{9} \mathrm{BrNO}_{2} \mathrm{~S}[\mathrm{M}+\mathrm{H}]^{+}$249.95, 251.95; found 249.78, 251.87 . 
Ethyl 5-amino-2-methylthiazole-4-carboxylate $(7)^{7}$

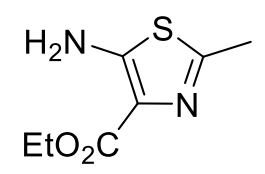

A mixture of ethyl 5-bromo-2-methylthiazole-4-carboxylate 6 (2.010 g, $8.0 \mathrm{mmol}$, 1 equiv), $\mathrm{Pd}_{2}(\mathrm{dba})_{3}$ (368 mg, $0.40 \mathrm{mmol}, 0.05$ equiv), ( \pm )-BINAP (495 mg, $0.80 \mathrm{mmol}, 0.10$ equiv), and cesium carbonate (3.685 g, $11.2 \mathrm{mmol}, 1.4$ equiv), and benzophenone imine (1.61 mL, $9.6 \mathrm{mmol}$, 1.2 equiv) in anhydrous toluene $(35 \mathrm{~mL})$ was sparged with $\mathrm{N}_{2}$ for 10 minutes. The reaction was heated to $80{ }^{\circ} \mathrm{C}$ for 20 hours, and then cooled to room temperature, diluted with EtOAc (100 mL) and filtered through Celite. Solvents were removed in vacuo to provide a dark red syrup. The intermediate ethyl 5-((diphenylmethylene)amino)-2-methylthiazole-4-carboxylate was carried on directly to hydrolysis. LRMS (ESI) calculated for $\mathrm{C}_{20} \mathrm{H}_{19} \mathrm{~N}_{2} \mathrm{O}_{2} \mathrm{~S}[\mathrm{M}+\mathrm{H}]^{+} 351.12$, found 350.87 .

Crude ethyl 5-((diphenylmethylene)amino)-2-methylthiazole-4-carboxylate was dissolved in 20 $\mathrm{mL} \mathrm{THF}$ and $5 \mathrm{~mL} 1 \mathrm{M} \mathrm{HCl}$. The mixture was stirred at room temperature for 4 hours, at which point ultra-performance liquid chromatography-mass spectrometry (UPLC-MS) analysis showed complete hydrolysis of the imine. The reaction was diluted with $50 \mathrm{~mL}$ EtOAc and then extracted with $1 \mathrm{M} \mathrm{HCl}(4 \times 50 \mathrm{~mL})$. The combined aqueous layers were neutralized to $\mathrm{pH}>12$ and then extracted with DCM $(5 \times 50 \mathrm{~mL})$. The combined DCM layers were washed with saturated aqueous $\mathrm{NaHCO}_{3}$, dried over $\mathrm{MgSO}_{4}$, filtered and concentrated to provide the title compound as a light yellow solid (1.011 g, 68\% yield over 2 steps). The product was sufficiently pure and did not require further purification.

${ }^{1} \mathrm{H}$ NMR $\left(500 \mathrm{MHz}, \mathrm{CDCl}_{3}\right) \delta 5.87(\mathrm{~s}, 2 \mathrm{H}), 4.38(\mathrm{q}, J=7.1 \mathrm{~Hz}, 2 \mathrm{H}), 2.53(\mathrm{~s}, 3 \mathrm{H}), 1.40(\mathrm{t}, J=7.1$ $\mathrm{Hz}, 3 \mathrm{H})$. LRMS (ESI) calculated for $\mathrm{C}_{7} \mathrm{H}_{11} \mathrm{~N}_{2} \mathrm{O}_{2} \mathrm{~S}[\mathrm{M}+\mathrm{H}]^{+}$187.05, found 186.87.

\section{Ethyl 2-methyl-5-(methylamino)thiazole-4-carboxylate (8)}<smiles>CCOC(=O)c1nc(C)sc1NC</smiles> 
General Procedure B was followed on $6.0 \mathrm{mmol}$ scale using ethyl 5-amino-2-methylthiazole-4carboxylate $7(1.120 \mathrm{~g})$ and iodomethane $(0.56 \mathrm{~mL})$ as the electrophile. ISCO flash chromatography (20 - 80\% EtOAC/hex, 18 minutes, $40 \mathrm{~g}$ silica) provided the title compound as a light yellow solid (502 mg, $41 \%$ yield).

${ }^{1} \mathrm{H}$ NMR (500 MHz, $\left.\mathrm{CDCl}_{3}\right) \delta 7.21(\mathrm{~s}, 1 \mathrm{H}), 4.36(\mathrm{q}, J=7.1 \mathrm{~Hz}, 2 \mathrm{H}), 3.00(\mathrm{~d}, J=5.2 \mathrm{~Hz}, 3 \mathrm{H}), 2.54$ (s, 3H), 1.38 (t, $J=7.1 \mathrm{~Hz}, 3 \mathrm{H})$. LRMS (ESI) calculated for $\mathrm{C}_{8} \mathrm{H}_{13} \mathrm{~N}_{2} \mathrm{O}_{2} \mathrm{~S}[\mathrm{M}+\mathrm{H}]^{+} 201.07$, found 200.87 .

Ethyl 5-((2-chloro-5-nitropyrimidin-4-yl)(methyl)amino)-2-methylthiazole-4-carboxylate (9)<smiles>CCOC(=O)c1nc(C)sc1N(C)c1nc(Cl)ncc1[N+](=O)[O-]</smiles>

General Procedure B was followed on 2.4 mmol scale using ethyl 2-methyl-5(methylamino)thiazole-4-carboxylate 8 (480 mg) as the nucleophile and 2,4-dichloro-5nitropyrimidine (746 mg, $3.85 \mathrm{mmol}, 1.6$ equiv) as the electrophile. The reaction was stirred for 5 hours. ISCO flash chromatography (20 - 80\% EtOAC/hex, 18 minutes, $40 \mathrm{~g}$ silica) provided the title compound as a yellow oil that partially solidified on standing (508 mg, 59\% isolated yield).

${ }^{1} \mathrm{H}$ NMR $\left(500 \mathrm{MHz}, \mathrm{CDCl}_{3}\right) \delta 8.59(\mathrm{~s}, 1 \mathrm{H}), 4.26(\mathrm{q}, J=7.1 \mathrm{~Hz}, 2 \mathrm{H}), 3.63(\mathrm{~s}, 3 \mathrm{H}), 2.73$ (s, 3H), $1.25(\mathrm{t}, J=7.1 \mathrm{~Hz}, 3 \mathrm{H})$. LRMS (ESI) calculated for $\mathrm{C}_{12} \mathrm{H}_{13} \mathrm{ClN}_{5} \mathrm{O}_{4} \mathrm{~S}[\mathrm{M}+\mathrm{H}]^{+}$358.04, found 357.77.

\section{6-chloro-2,4-dimethyl-4,9-dihydro-10H-pyrimido[5,4-b]thiazolo[5,4-e][1,4]diazepin-10-one} (S-1)<smiles>Cc1nc2c(s1)N(C)c1nc(Cl)ncc1NC2=O</smiles>

General Procedure C was followed on $1.4 \mathrm{mmol}$ scale using ethyl 5-((2-chloro-5-nitropyrimidin4-yl)(methyl)amino)-2-methylthiazole-4-carboxylate (499 mg). The reaction was cooled to room temperature and residual iron was removed with a magnetic wand. The crude reaction mixture was poured into a beaker with $150 \mathrm{~mL}$ water and stirred at room temperature for 30 minutes. The 
resulting precipitate was collected by suction filtration, washing with $\sim 100 \mathrm{~mL}$ water, and dried in vacuo to provide the title compound as a beige solid (258 $\mathrm{mg}, 65 \%$ isolated yield).

${ }^{1} \mathrm{H}$ NMR (500 MHz, MeOD) $\delta 8.09$ (s, 1H), 3.46 (s, 3H), 2.60 (s, 3H). LRMS (ESI) calculated for $\mathrm{C}_{10} \mathrm{H}_{9} \mathrm{ClN}_{5} \mathrm{OS}[\mathrm{M}+\mathrm{H}]^{+} 282.02$, found 282.07 .

6-chloro-2,4,9-trimethyl-4,9-dihydro-10H-pyrimido[5,4-b]thiazolo[5,4-e][1,4]diazepin-10one (10)<smiles>Cc1nc2c(s1)N(C)c1nc(Cl)ncc1N(C)C2=O</smiles>

General Procedure D was followed on $0.95 \mathrm{mmol}$ scale, using S-1 (267 mg, $0.95 \mathrm{mmol}$, 1.0 equiv) and iodomethane $(0.130 \mathrm{~mL}, 2.0 \mathrm{mmol}, 2.1$ equiv). The reaction was stirred for 2 hours. The reaction was then quenched with water $(20 \mathrm{~mL})$ and extracted with EtOAc $(4 \times 40 \mathrm{~mL})$. The combined organic layers were washed twice with water, once with brine, dried over $\mathrm{MgSO}_{4}$, filtered and concentrated. ISCO flash chromatography (24 g silica, 0-10\% MeOH/DCM, 14 min gradient) provided the title compound as a light yellow solid (219.7 mg, $74 \%$ yield).

${ }^{1} \mathrm{H}$ NMR $\left(500 \mathrm{MHz}, \mathrm{CDCl}_{3}\right) \delta 8.23(\mathrm{~s}, 1 \mathrm{H}), 3.45$ (s, 3H), 3.43 (s, 3H), 2.62 (s, 3H). LRMS (ESI) calculated for $\mathrm{C}_{11} \mathrm{H}_{11} \mathrm{ClN}_{5} \mathrm{OS}[\mathrm{M}+\mathrm{H}]^{+} 296.04$, found 295.77 .

6-chloro-9-ethyl-2,4-dimethyl-4,9-dihydro-10H-pyrimido[5,4-b]thiazolo[5,4-e][1,4]diazepin10-one (S-2)<smiles>CCN1C(=O)c2nc(C)sc2N(C)c2nc(Cl)ncc21</smiles>

To a suspension of 6-chloro-2,4-dimethyl-4,9-dihydro-10H-pyrimido[5,4-b]thiazolo[5,4e][1,4]diazepin-10-one $\mathbf{S}-1$ (29.5 mg, $0.10 \mathrm{mmol}, 1.0$ equiv) and iodoethane $(20.0 \mu \mathrm{L}, 0.25 \mathrm{mmol}$, 2.5 equiv) in anhydrous $\mathrm{DMF}(1.5 \mathrm{~mL})$ at $0{ }^{\circ} \mathrm{C}, \mathrm{NaH}(10.1 \mathrm{mg}, 0.22 \mathrm{mmol}, 2.2$ equiv, 60\% 
dispersion in mineral oil) was added in a single portion. The reaction was stirred overnight, and UPLC-MS analysis showed complete consumption of starting material. The reaction was then quenched with water $(10 \mathrm{~mL})$ and extracted with EtOAc $(4 \times 10 \mathrm{~mL})$. The combined organic layers were washed twice with water, once with brine, dried over $\mathrm{MgSO}_{4}$, filtered and concentrated. ISCO flash chromatography (12 g silica, 0-10\% MeOH/DCM, 10 min gradient) provided the title compound as an amber oil (35.0 mg, quantitative yield).

LRMS (ESI) calculated for $\mathrm{C}_{12} \mathrm{H}_{13} \mathrm{ClN}_{5} \mathrm{OS}[\mathrm{M}+\mathrm{H}]^{+} 310.05$, found 309.77 .

Synthesis of unsubstituted thiazole core

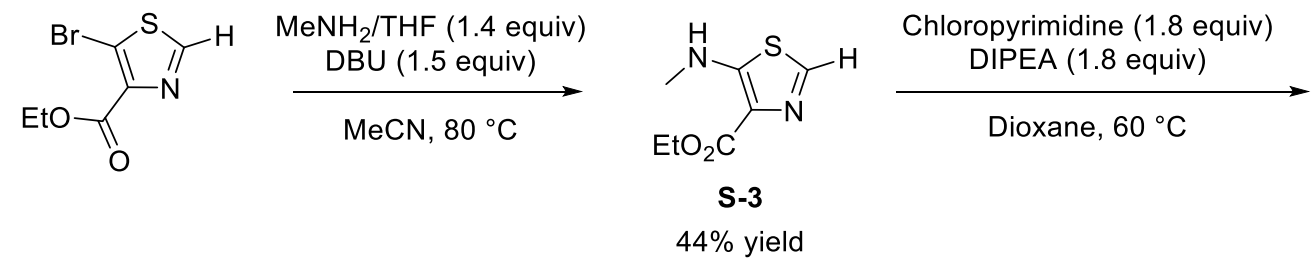

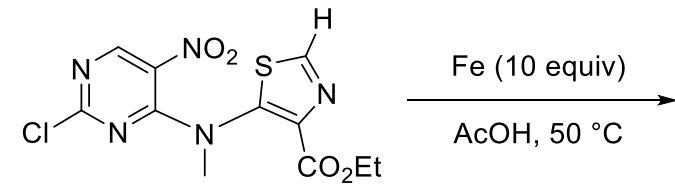

S-4

$45 \%$ yield

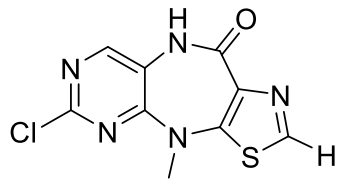

S-5

$52 \%$ yield

\section{Ethyl 5-(methylamino)thiazole-4-carboxylate (S-3) ${ }^{8}$}<smiles>CCOC(=O)c1nccs1</smiles>

To a solution of ethyl 5-bromothiazole-4-carboxylate $(1.191 \mathrm{~g}, 5.0 \mathrm{mmol}, 1.0$ equiv) in anhydrous acetonitrile $(12 \mathrm{~mL})$ were added methylamine (2.0 M in THF, $3.6 \mathrm{~mL}, 7.2 \mathrm{mmol}, 1.44$ equiv) and DBU (1.120 mL, $7.5 \mathrm{mmol}, 1.5$ equiv). The mixture was heated to $80{ }^{\circ} \mathrm{C}$ for 4 hours, and then cooled to room temperature. The reaction was diluted with $50 \mathrm{~mL}$ each EtOAc and water. The organic layer was removed and the aqueous was extracted with EtOAc $(3 \times 40 \mathrm{~mL})$. The combined 
organic layers were washed with brine, dried over $\mathrm{MgSO}_{4}$, filtered and concentrated. ISCO flash chromatography ( $24 \mathrm{~g}$ silica, 25 to $80 \%$ EtOAc/hexanes gradient, 18 minutes) provided the title compound as a white solid (412 $\mathrm{mg}, 44 \%$ yield).

${ }^{1} \mathrm{H}$ NMR $\left(500 \mathrm{MHz}, \mathrm{CDCl}_{3}\right) \delta 7.85(\mathrm{~d}, J=1.0 \mathrm{~Hz}, 1 \mathrm{H}), 7.31(\mathrm{~s}, 1 \mathrm{H}), 4.37(\mathrm{q}, J=7.1 \mathrm{~Hz}, 2 \mathrm{H}), 3.05$ $(\mathrm{d}, J=5.1 \mathrm{~Hz}, 3 \mathrm{H}), 1.41(\mathrm{t}, J=7.0 \mathrm{~Hz}, 3 \mathrm{H})$. LRMS (ESI) calculated for $\mathrm{C}_{7} \mathrm{H}_{11} \mathrm{~N}_{2} \mathrm{O}_{2} \mathrm{~S}[\mathrm{M}+\mathrm{H}]^{+}$ 187.05, found 187.19.

Ethyl 5-((2-chloro-5-nitropyrimidin-4-yl)(methyl)amino)thiazole-4-carboxylate (S-4)<smiles>CCOC(=O)c1nc(C)sc1N(C)c1nc(Cl)ncc1[N+](=O)[O-]</smiles>

A mixture of ethyl 5-(methylamino)thiazole-4-carboxylate $\mathbf{S - 3}$ (377 mg, $2.0 \mathrm{mmol}, 1.0$ equiv), 2,4-dichloro-5-nitropyrimidine (696 mg, $3.6 \mathrm{mmol}, 1.8$ equiv), and DIPEA (0.65 mL, $3.6 \mathrm{mmol}$, 1.8 equiv) in dioxane $(8 \mathrm{~mL})$ was heated to $80{ }^{\circ} \mathrm{C}$ for 2 days. The reaction was diluted with $50 \mathrm{~mL}$ EtOAc and $100 \mathrm{~mL}$ water. The organic layer was removed and the aqueous was extracted with EtOAc $(5 \times 40 \mathrm{~mL})$. The combined organic layers were washed twice with water and twice with brine, dried over $\mathrm{MgSO}_{4}$, filtered and concentrated. ISCO flash chromatography (40 g silica, 20 to $80 \%$ EtOAc/hexanes gradient, 18 minutes) provided the title compound as a dark red solid (311 $\mathrm{mg}, 45 \%$ isolated yield).

${ }^{1} \mathrm{H}$ NMR (500 MHz, $\left.\mathrm{CDCl}_{3}\right) \delta 8.76(\mathrm{~s}, 1 \mathrm{H}), 8.62(\mathrm{~s}, 1 \mathrm{H}), 4.28(\mathrm{q}, J=7.1 \mathrm{~Hz}, 2 \mathrm{H}), 3.67(\mathrm{~s}, 3 \mathrm{H})$, $1.29(\mathrm{t}, J=7.1 \mathrm{~Hz}, 3 \mathrm{H})$. LRMS (ESI) calculated for $\mathrm{C}_{11} \mathrm{H}_{11} \mathrm{ClN}_{5} \mathrm{O}_{4} \mathrm{~S}[\mathrm{M}+\mathrm{H}]^{+}$344.02, found 344.07 $(\mathrm{M}+\mathrm{H})^{+}$.

6-chloro-4-methyl-4,9-dihydro-10H-pyrimido[5,4-b]thiazolo[5,4-e][1,4]diazepin-10-one (S5)<smiles></smiles> 
General Procedure C was followed on $0.90 \mathrm{mmol}$ scale using ethyl 5-((2-chloro-5-nitropyrimidin4-yl)(methyl)amino)thiazole-4-carboxylate $\mathbf{S - 4}$ (311 mg) for 16 hours. The crude reaction mixture was poured into a beaker with $40 \mathrm{~mL}$ water and stirred at room temperature for 30 minutes. The resulting precipitate was collected by suction filtration, washing with $\sim 100 \mathrm{~mL}$ water, and dried in vacuo to provide the title compound as a light tan solid (127.4 mg, 52\% yield).

LRMS (ESI) calculated for $\mathrm{C}_{9} \mathrm{H}_{7} \mathrm{~N}_{5} \mathrm{OS}[\mathrm{M}+\mathrm{H}]^{+}$268.01, found 268.07.

Synthesis of 2-ethylthiazole tricyclic core
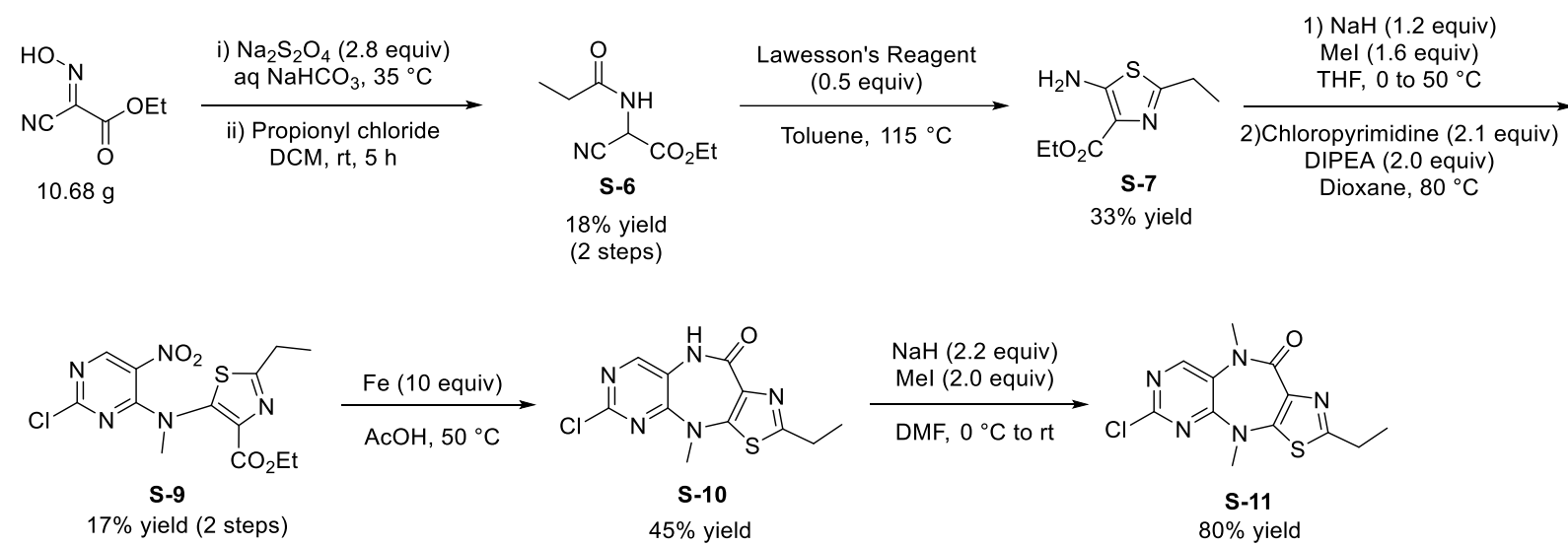

Ethyl 2-cyano-2-propionamidoacetate (S-6) ${ }^{9}$<smiles>CCOC(=O)C(C#N)NC(=O)CC</smiles>

To a stirred solution of ethyl cyano(hydroxyimino)acetate (8.590 g, $60 \mathrm{mmol}, 1.0$ equiv) in 180 mL saturated aqueous $\mathrm{NaHCO}_{3}$, sodium hydrosulfite (34.3 g, $168 \mathrm{mmol}, 2.8$ equiv) was added portion-wise over 5 minutes. The reaction was heated to $35^{\circ} \mathrm{C}$ for 1 hour, and then cooled to room temperature and extracted with DCM $(5 \times 100 \mathrm{~mL})$. The combined organic layers were washed with brine, dried over $\mathrm{MgSO}_{4}$, filtered and concentrated to provide a clear, orange oil $(1.790 \mathrm{~g}, 23 \%$ yield). The crude ethyl cyanoglycine was further reacted without purification. 
${ }^{1} \mathrm{H}$ NMR $\left(500 \mathrm{MHz}, \mathrm{CDCl}_{3}\right) \delta 4.43(\mathrm{~s}, 1 \mathrm{H}), 4.34(\mathrm{q}, J=7.2 \mathrm{~Hz}, 2 \mathrm{H}), 1.95(\mathrm{~s}, 2 \mathrm{H}), 1.35(\mathrm{t}, J=7.1$ $\mathrm{Hz}, 3 \mathrm{H})$.

To a solution of ethyl cyanoglycine (512 mg, $4.0 \mathrm{mmol}, 1.0$ equiv) in DCM (8 mL), propionyl chloride $(0.49 \mathrm{~mL}, 5.6 \mathrm{mmol}, 1.4$ equiv) was added dropwise, followed by triethylamine $(0.89 \mathrm{~mL}$, $6.4 \mathrm{mmol}, 1.6$ equiv). The reaction was stirred at room temperature overnight, and then diluted with DCM and saturated aqueous $\mathrm{NaHCO}_{3}(25 \mathrm{~mL}$ each). The organic layer was removed, and the aqueous layer extracted with DCM $(2 \times 20 \mathrm{~mL})$. The combined organic layers were washed with brine, dried over $\mathrm{MgSO}_{4}$, filtered and concentrated. ISCO flash chromatography (40 g silica, 50 to $80 \% \mathrm{EtOAc/hexanes} \mathrm{gradient)} \mathrm{provided} \mathrm{the} \mathrm{title} \mathrm{compound} \mathrm{as} \mathrm{an} \mathrm{off-white} \mathrm{solid} \mathrm{(577} \mathrm{mg}, 78 \%$ yield).

${ }^{1} \mathrm{H}$ NMR $\left(500 \mathrm{MHz}, \mathrm{CDCl}_{3}\right) \delta 6.30(\mathrm{~s}, 1 \mathrm{H}), 5.54(\mathrm{~d}, J=7.7 \mathrm{~Hz}, 1 \mathrm{H}), 4.36(\mathrm{q}, J=7.1 \mathrm{~Hz}, 2 \mathrm{H}), 2.35$ $(\mathrm{q}, J=7.6 \mathrm{~Hz}, 2 \mathrm{H}), 1.37(\mathrm{t}, J=7.1 \mathrm{~Hz}, 3 \mathrm{H}), 1.20(\mathrm{t}, J=7.6 \mathrm{~Hz}, 3 \mathrm{H})$.

\section{Ethyl 5-amino-2-ethylthiazole-4-carboxylate (S-7)}<smiles>CCOC(=O)c1nc(CC)sc1N</smiles>

To a $50 \mathrm{~mL}$ round-bottom flask were added ethyl 2-cyano-2-propionamidoacetate $\mathbf{S - 6}$ (554 mg, $3.0 \mathrm{mmol}, 1.0$ equiv), anhydrous toluene (16 mL), Lawesson's reagent (739 mg, $1.8 \mathrm{mmol}, 0.6$ equiv), and $3 \AA$ molecular sieves (powder, $130 \mathrm{mg}$ ). The suspension was flushed with nitrogen, sealed and heated overnight. The reaction was then cooled to room temperature, filtered and washed with $50 \mathrm{~mL}$ EtOAc. The title product was isolated by acidic extraction (extracted crude material 4x25 mL $1 \mathrm{M} \mathrm{HCl}$, neutralized aqueous component, and extracted 4x50 mL EtOAc) and concentrated to provide the title compound as a light brown solid (202 mg, 33\% yield). The material was carried on to the next step without further purification.

LRMS (ESI) calculated for $\mathrm{C}_{8} \mathrm{H}_{13} \mathrm{~N}_{2} \mathrm{O}_{2} \mathrm{~S}[\mathrm{M}+\mathrm{H}]^{+}$201.07, found 200.26. 
Ethyl 2-ethyl-5-(methylamino)thiazole-4-carboxylate (S-8)<smiles>CCOC(=O)c1nc(CC)sc1NC</smiles>

General Procedure B was followed on $1.0 \mathrm{mmol}$ scale using ethyl 5-amino-2-ethylthiazole-4carboxylate S-7 (199 $\mathrm{mg})$ and iodomethane as the electrophile. Modification: the reaction was heated to $50{ }^{\circ} \mathrm{C}$ for 15 hours. ISCO flash chromatography (12 g silica, 25-80\% EtOAc/hexanes, $16 \mathrm{~min}$ gradient) provided the title compound as a clear yellow oil (70.1 mg, 33\% yield).

${ }^{1} \mathrm{H}$ NMR (500 MHz, $\left.\mathrm{CDCl}_{3}\right) \delta 7.24(\mathrm{~s}, 1 \mathrm{H}), 4.37(\mathrm{q}, J=7.1 \mathrm{~Hz}, 2 \mathrm{H}), 3.02(\mathrm{~d}, J=5.1 \mathrm{~Hz}, 3 \mathrm{H}), 2.89$ $(\mathrm{q}, J=7.6 \mathrm{~Hz}, 2 \mathrm{H}), 1.39(\mathrm{t}, J=7.1 \mathrm{~Hz}, 3 \mathrm{H}), 1.31(\mathrm{t}, J=7.6 \mathrm{~Hz}, 3 \mathrm{H})$. LRMS (ESI) calculated for $\mathrm{C}_{9} \mathrm{H}_{15} \mathrm{~N}_{2} \mathrm{O}_{2} \mathrm{~S}[\mathrm{M}+\mathrm{H}]^{+}$215.08, found 215.28.

Ethyl 5-((2-chloro-5-nitropyrimidin-4-yl)(methyl)amino)-2-ethylthiazole-4-carboxylate (S9)<smiles>CCOC(=O)c1nc(CC)sc1N(C)c1nc(Cl)ncc1[N+](=O)[O-]</smiles>

A mixture of ethyl 2-ethyl-5-(methylamino)thiazole-4-carboxylate $\mathbf{S - 8}$ (70.1 mg, $0.33 \mathrm{mmol}, 1.0$ equiv), 2,4-dichloro-5-nitropyrimidine (135.8 mg, $0.70 \mathrm{mmol}, 2.1$ equiv), and DIPEA (0.11 mL, $0.60 \mathrm{mmol}, 1.8$ equiv) in dioxane $(1.5 \mathrm{~mL})$ was heated to $80{ }^{\circ} \mathrm{C}$ for 22 hours. ISCO flash chromatography (12 g silica, 20-80\% EtOAc/hexanes, $15 \mathrm{~min}$ gradient) provided the title compound (66.0 mg, 53\% yield) as a clear yellow-orange oil. ${ }^{1} \mathrm{H} \mathrm{NMR}\left(500 \mathrm{MHz}, \mathrm{CDCl}_{3}\right) \delta 8.58$ $(\mathrm{s}, 1 \mathrm{H}), 4.26(\mathrm{q}, J=7.1 \mathrm{~Hz}, 2 \mathrm{H}), 3.63(\mathrm{~s}, 3 \mathrm{H}), 3.04(\mathrm{q}, J=7.6 \mathrm{~Hz}, 2 \mathrm{H}), 1.40$ (t, $J=7.6 \mathrm{~Hz}, 3 \mathrm{H})$, $1.25(\mathrm{t}, J=7.1 \mathrm{~Hz}, 3 \mathrm{H})$. LRMS (ESI) calculated for $\mathrm{C}_{13} \mathrm{H}_{15} \mathrm{ClN}_{5} \mathrm{O}_{4} \mathrm{~S}[\mathrm{M}+\mathrm{H}]^{+} 372.05$, found 372.07 . 

one (S-10)<smiles>CCc1nc2c(s1)N(C)c1nc(Cl)ncc1NC2=O</smiles>

General Procedure C was followed on 0.177 mmol scale using ethyl 5-((2-chloro-5nitropyrimidin-4-yl)(methyl)amino)-2-ethylthiazole-4-carboxylate S-9 (66.0 mg, 0.177 mmol, 1.0 equiv). Modified isolation: after the precipitate settled, the supernatant was removed by decantation. The precipitate was washed with water $\left(2 \times 3 \mathrm{~mL}\right.$, decantation) and dried at $45{ }^{\circ} \mathrm{C}$ under a stream of $\mathrm{N}_{2}$ to provide the title compound as a yellow solid ( $21.3 \mathrm{mg}, 45 \%$ yield).

${ }^{1} \mathrm{H}$ NMR $\left(500 \mathrm{MHz}, \mathrm{CDCl}_{3}\right) \delta 9.15(\mathrm{~s}, 1 \mathrm{H}), 8.17$ (s, 1H), 3.45 (s, 3H), 2.93 (s, 2H), 1.33 (s, 3H) (signals broadened due to paramagnetism of residual Fe). LRMS (ESI) calculated for $\mathrm{C}_{11} \mathrm{H}_{11} \mathrm{ClN}_{5} \mathrm{OS}[\mathrm{M}+\mathrm{H}]^{+} 296.04$, found 296.07.

6-chloro-2-ethyl-4,9-dimethyl-4,9-dihydro-10H-pyrimido[5,4-b]thiazolo[5,4-e][1,4]diazepin10-one (S-11)<smiles>CCc1nc2c(s1)N(C)c1nc(Cl)ncc1N(C)C2=O</smiles>

General Procedure D was followed on $0.20 \mathrm{mmol}$ scale using 6-chloro-2-ethyl-4-methyl-4,9dihydro-10H-pyrimido[5,4-b]thiazolo[5,4-e][1,4]diazepin-10-one $\quad \mathbf{S - 1 0} \quad(63.0 \quad \mathrm{mg}) \quad$ and iodomethane $(25.0 \mu \mathrm{L})$ as the electrophile, for a reaction time of 3 hours. The reaction was then quenched with water $(5 \mathrm{~mL})$ and extracted with EtOAc $(4 \times 5 \mathrm{~mL})$. The combined organic layers were washed three times with water, once with brine, dried over $\mathrm{MgSO}_{4}$, filtered and concentrated. ISCO flash chromatography (12 g silica, 0-5\% MeOH/DCM, 12 min gradient) provided the title compound as a light yellow solid (49.8 mg, $80 \%$ yield). 
${ }^{1} \mathrm{H}$ NMR (500 MHz, DMSO-d $) \delta 8.58(\mathrm{~s}, 1 \mathrm{H}), 3.37$ (s, 3H), $3.31(\mathrm{~s}, 3 \mathrm{H}), 2.88(\mathrm{q}, J=7.5 \mathrm{~Hz}, 2 \mathrm{H})$, $1.24(\mathrm{t}, J=7.5 \mathrm{~Hz}, 3 \mathrm{H})$. LRMS (ESI) calculated for $\mathrm{C}_{12} \mathrm{H}_{13} \mathrm{ClN}_{5} \mathrm{OS}[\mathrm{M}+\mathrm{H}]^{+} 310.05$, found 309.87 $(\mathrm{M}+\mathrm{H})^{+}$.

Synthesis of 2-methylthiazole-4-N-ethyl core

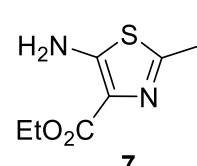

7

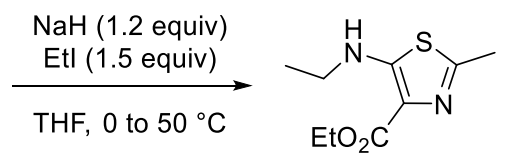

S-12
Chloropyrimidine ( 2.1 equiv) DIPEA ( 2.5 equiv)

Dioxane, $80^{\circ} \mathrm{C}$ $24 \%$ yield

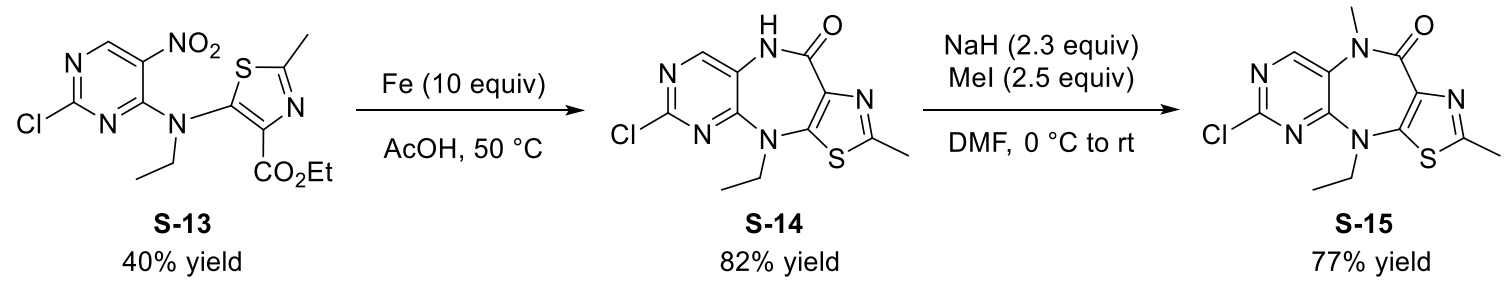

Ethyl 5-(ethylamino)-2-methylthiazole-4-carboxylate (S-12)<smiles>CCNc1sc(C)nc1C(=O)OCC</smiles>

General Procedure B was followed on $1.03 \mathrm{mmol}$ scale using ethyl 5-amino-2-methylthiazole-4carboxylate $7(191.5 \mathrm{mg})$ as nucleophile and iodoethane $(0.120 \mathrm{~mL})$ as the electrophile. The reaction was heated to $50{ }^{\circ} \mathrm{C}$ for 15 hours. ISCO flash chromatography (12 g silica, 25-80\% EtOAc/hexanes, $16 \mathrm{~min}$ gradient) provided the title compound as a red-orange oil (52.9 mg, 24\% yield).

${ }^{1} \mathrm{H}$ NMR (500 MHz, $\left.\mathrm{CDCl}_{3}\right) \delta 7.24(\mathrm{~s}, 1 \mathrm{H}), 4.37(\mathrm{q}, J=7.1 \mathrm{~Hz}, 2 \mathrm{H}), 3.24(\mathrm{qd}, J=7.2,5.6 \mathrm{~Hz}$, 2H), $2.55(\mathrm{~s}, 3 \mathrm{H}), 1.39(\mathrm{t}, J=7.1 \mathrm{~Hz}, 3 \mathrm{H}), 1.31(\mathrm{t}, J=7.2 \mathrm{~Hz}, 3 \mathrm{H})$. LRMS (ESI) calculated for $\mathrm{C}_{9} \mathrm{H}_{15} \mathrm{~N}_{2} \mathrm{O}_{2} \mathrm{~S}[\mathrm{M}+\mathrm{H}]^{+}$215.08, found 215.18. 
Ethyl 5-((2-chloro-5-nitropyrimidin-4-yl)(ethyl)amino)-2-methylthiazole-4-carboxylate (S13)<smiles>CCOC(=O)c1nc(C)sc1N(CC)c1nc(Cl)ncc1[N+](=O)[O-]</smiles>

A solution of ethyl 5-(ethylamino)-2-methylthiazole-4-carboxylate S-12 (52.9 mg, $0.25 \mathrm{mmol}, 1.0$ equiv), 2,4-dichloro-5-nitropyrimidine (105 mg, $0.54 \mathrm{mmol}, 2.1$ equiv), and DIPEA (0.08 mL, $0.45 \mathrm{mmol}, 1.8$ equiv) in dioxane $(1.0 \mathrm{~mL})$ was heated to $80{ }^{\circ} \mathrm{C}$ for 22 hours. ISCO flash chromatography (12 g silica, 20-80\% EtOAc/hexanes, $15 \mathrm{~min}$ gradient) provided the title compound as a yellow-orange waxy solid (38.0 mg, $40 \%$ yield).

${ }^{1} \mathrm{H}$ NMR $\left(500 \mathrm{MHz}, \mathrm{CDCl}_{3}\right) \delta 8.55(\mathrm{~s}, 1 \mathrm{H}), 4.31-4.05(\mathrm{~m}, 4 \mathrm{H}), 2.72(\mathrm{~s}, 3 \mathrm{H}), 1.29(\mathrm{t}, J=7.1 \mathrm{~Hz}$, $3 \mathrm{H}), 1.23(\mathrm{t}, J=7.1 \mathrm{~Hz}, 3 \mathrm{H})$. LRMS (ESI) calculated for $\mathrm{C}_{13} \mathrm{H}_{15} \mathrm{ClN}_{5} \mathrm{O}_{4} \mathrm{~S}[\mathrm{M}+\mathrm{H}]^{+} 372.05$, found 372.07 .

6-chloro-4-ethyl-2-methyl-4,9-dihydro-10H-pyrimido[5,4-b]thiazolo[5,4-e][1,4]diazepin-10one (S-14)<smiles>CCN1c2nc(Cl)ncc2NC(=O)c2nc(C)sc21</smiles>

General Procedure C was followed on $0.10 \mathrm{mmol}$ scale using ethyl 5-((2-chloro-5-nitropyrimidin4-yl)(ethyl)amino)-2-methylthiazole-4-carboxylate S-13 (38.0 mg). Modified isolation: the reaction was diluted with $5 \mathrm{~mL}$ water, residual Fe was removed with a magnet, and then the aqueous component was extracted with DCM $(4 \times 8 \mathrm{~mL})$ The combined DCM layers were washed with brine, dried over $\mathrm{MgSO}_{4}$, filtered and concentrated to provide the title compound as a light yellow solid (24.5 mg, $82 \%$ yield). 
${ }^{1} \mathrm{H}$ NMR (500 MHz, $\left.\mathrm{CDCl}_{3}\right) \delta 9.21(\mathrm{~s}, 1 \mathrm{H}), 8.13(\mathrm{~s}, 1 \mathrm{H}), 4.00(\mathrm{q}, J=7.1 \mathrm{~Hz}, 2 \mathrm{H}), 2.61(\mathrm{~s}, 3 \mathrm{H})$, $1.43(\mathrm{t}, J=7.1 \mathrm{~Hz}, 3 \mathrm{H})$. LRMS (ESI) calculated for $\mathrm{C}_{11} \mathrm{H}_{11} \mathrm{ClN}_{5} \mathrm{OS}[\mathrm{M}+\mathrm{H}]^{+} 296.04$, found 296.07.

6-chloro-4-ethyl-2,9-dimethyl-4,9-dihydro-10H-pyrimido[5,4-b]thiazolo[5,4-e][1,4]diazepin10-one (S-15)<smiles>CCN1c2nc(Cl)ncc2N(C)C(=O)c2nc(C)sc21</smiles>

General Procedure D was followed on $0.13 \mathrm{mmol}$ scale using 6-chloro-4-ethyl-2-methyl-4,9dihydro-10H-pyrimido[5,4-b]thiazolo[5,4-e][1,4]diazepin-10-one $\quad \mathbf{S - 1 4} \quad(38.4 \quad \mathrm{mg})$ and iodomethane (20.0 $\mu \mathrm{L}, 2.5$ equiv) as the electrophile, with a reaction time of 1 hour. The reaction was then quenched with water $(5 \mathrm{~mL})$ and extracted with EtOAc $(5 \times 8 \mathrm{~mL})$. The combined organic layers were washed twice with water, once with brine, dried over $\mathrm{MgSO}_{4}$, filtered and concentrated. ISCO flash chromatography (12 g silica, 0-10\% MeOH/DCM, $12 \mathrm{~min}$ gradient) provided the title compound as a yellow oil (31.0 mg, $77 \%$ yield).

LRMS (ESI) calculated for $\mathrm{C}_{12} \mathrm{H}_{13} \mathrm{ClN}_{5} \mathrm{OS}[\mathrm{M}+\mathrm{H}]^{+} 310.05$, found 309.77 .

Synthesis of 2-methylthiazole-4-N-propyl core

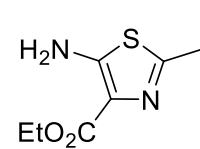

7

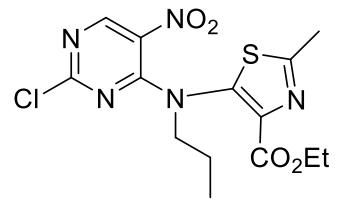

S-17

$56 \%$ yield

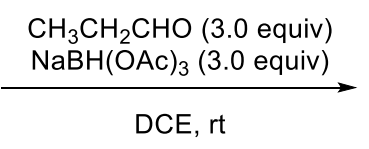

DCE, rt

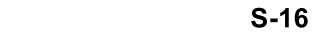

$43 \%$ yield

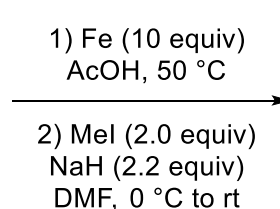

$\mathrm{DMF}, 0^{\circ} \mathrm{C}$ to $\mathrm{rt}$

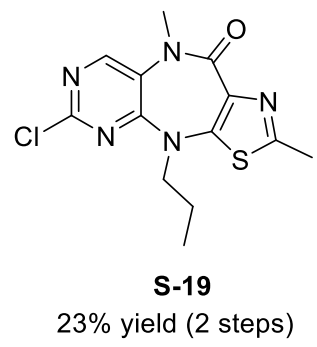

$\mathrm{NaH}$ (1.3 equiv)

Chloropyrimidine (1.8 equiv)

THF, $0^{\circ} \mathrm{C}$ to $\mathrm{rt}$ 
Ethyl 2-methyl-5-(propylamino)thiazole-4-carboxylate $(\mathrm{S}-16)^{10}$

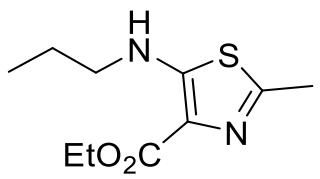

To a solution of ethyl 5-amino-2-methylthiazole-4-carboxylate 7 (702 mg, $3.5 \mathrm{mmol}, 1.0$ equiv) and propionaldehyde $\left(0.80 \mathrm{~mL}, 10.5 \mathrm{mmol}, 3.0\right.$ equiv) in DCE $(18 \mathrm{~mL}), \mathrm{NaBH}(\mathrm{OAc})_{3}(2.260 \mathrm{~g}$, $10.5 \mathrm{mmol}, 3.0$ equiv) was added portion-wise over 3 minutes at room temperature. The reaction was stirred for 4 hours, and then quenched with sat. $\mathrm{NaHCO}_{3}(100 \mathrm{~mL})$. The aqueous component was extracted with DCM $(3 \times 30 \mathrm{~mL})$; the combined organic layers were washed with water and brine, dried over $\mathrm{MgSO}_{4}$, filtered and concentrated. ISCO flash chromatography (24 g silica, 20$70 \%$ EtOAc/Hex, $15 \mathrm{~min}$ ) provided the title compound as a yellow oil (349 $\mathrm{mg}, 43 \%$ yield).

${ }^{1} \mathrm{H} \mathrm{NMR}\left(500 \mathrm{MHz}, \mathrm{CDCl}_{3}\right) \delta 7.33(\mathrm{~s}, 1 \mathrm{H}), 4.37$ (q, J = 7.1 Hz, 2H), $3.17(\mathrm{td}, \mathrm{J}=7.0,5.8 \mathrm{~Hz}, 2 \mathrm{H})$, 2.54 (s, 3H), 1.70 (h, J = 7.4 Hz, 2H), 1.39 (t, J = 7.1 Hz, 3H), 0.99 (t, J = 7.4 Hz, 3H). LRMS (ESI) calculated for $\mathrm{C}_{10} \mathrm{H}_{17} \mathrm{~N}_{2} \mathrm{O}_{2} \mathrm{~S}[\mathrm{M}+\mathrm{H}]^{+} 229.10$, found 229.08 .

Ethyl 5-((2-chloro-5-nitropyrimidin-4-yl)(propyl)amino)-2-methylthiazole-4-carboxylate (S17)<smiles>CCCN(c1nc(Cl)ncc1[N+](=O)[O-])c1sc(C)nc1C(=O)OCC</smiles>

General Procedure B was followed on $1.5 \mathrm{mmol}$ scale using ethyl 2-methyl-5(propylamino)thiazole-4-carboxylate $\mathbf{S - 1 6}(349 \mathrm{mg})$ as nucleophile and 2,4-dichloro-5nitropyrimidine (551 mg, 1.8 equiv) was added. The reaction was warmed to room temperature over 1 hour, and then heated to $68{ }^{\circ} \mathrm{C}$ for 6 hours. ISCO flash chromatography (20-70\% EtOAC/hex, 17 minutes, $24 \mathrm{~g}$ silica) provided the title compound as a red oil (327 $\mathrm{mg}$, 56\% isolated yield). 
${ }^{1} \mathrm{H}$ NMR $\left(500 \mathrm{MHz}, \mathrm{CDCl}_{3}\right) \delta 8.55(\mathrm{~s}, 1 \mathrm{H}), 4.23(\mathrm{q}, \mathrm{J}=7.2 \mathrm{~Hz}, 2 \mathrm{H}), 4.10-3.91(\mathrm{~m}, 2 \mathrm{H}), 2.72(\mathrm{~s}$, $3 \mathrm{H}), 1.79-1.65(\mathrm{~m}, 2 \mathrm{H}), 1.23(\mathrm{t}, \mathrm{J}=7.1 \mathrm{~Hz}, 3 \mathrm{H}), 0.97$ (t, J = 7.4 Hz, 3H). LRMS (ESI) calculated for $\mathrm{C}_{14} \mathrm{H}_{17} \mathrm{ClN}_{5} \mathrm{O}_{4} \mathrm{~S}[\mathrm{M}+\mathrm{H}]^{+} 386.07$, found 385.87.

6-chloro-2-methyl-4-propyl-4,9-dihydro-10H-pyrimido[5,4-b]thiazolo[5,4-e][1,4]diazepin10-one (S-18)<smiles>CCCN1c2nc(Cl)ncc2NC(=O)c2nc(C)sc21</smiles>

General Procedure C was followed on 0.84 mmol scale using ethyl 5-((2-chloro-5-nitropyrimidin4-yl)(propyl)amino)-2-methylthiazole-4-carboxylate S-17 (327 mg) for 18 hours. Modified isolation: After removal of iron, the crude reaction mixture was poured into $100 \mathrm{~mL}$ water and extracted with DCM $(5 \times 20 \mathrm{~mL})$. The combined organic layers were washed twice with sat. aqueous $\mathrm{NaHCO}_{3}$ and then brine, dried over $\mathrm{MgSO}_{4}$, filtered and concentrated to provide the title compound as a yellow oil (245 $\mathrm{mg}, 94 \%$ yield).

${ }^{1} \mathrm{H}$ NMR $\left(500 \mathrm{MHz}, \mathrm{CDCl}_{3}\right) \delta 8.09$ (s, 1H), 3.93 (t, J = 7.2 Hz, 2H), 2.62 (s, 3H), 1.84 (h, J = 7.4 $\mathrm{Hz}, 2 \mathrm{H}), 1.00(\mathrm{t}, \mathrm{J}=7.4 \mathrm{~Hz}, 3 \mathrm{H})$. LRMS (ESI) calculated for $\mathrm{C}_{12} \mathrm{H}_{13} \mathrm{ClN}_{5} \mathrm{OS}[\mathrm{M}+\mathrm{H}]^{+} 310.05$, found 309.87 .

6-chloro-2,9-dimethyl-4-propyl-4,9-dihydro-10H-pyrimido[5,4-b]thiazolo[5,4e][1,4]diazepin-10-one (S-19)<smiles>CCCN1c2nc(Cl)ncc2N(C)C(=O)c2nc(C)sc21</smiles> 
To a suspension of 6-chloro-2-methyl-4-propyl-4,9-dihydro-10H-pyrimido[5,4-b]thiazolo[5,4e][1,4]diazepin-10-one $\mathbf{S - 1 8}$ (245 mg, $0.80 \mathrm{mmol}, 1.0$ equiv) and iodomethane (0.10 $\mathrm{mL}, 1.60$ mmol, 2.0 equiv) in anhydrous DMF $(10 \mathrm{~mL})$ at $0{ }^{\circ} \mathrm{C}, \mathrm{NaH}(70 \mathrm{mg}, 1.76 \mathrm{mmol}, 2.2$ equiv, 60\% dispersion in mineral oil) was added in a single portion. The reaction was stirred for 1.5 hours. The reaction was then quenched with water $(20 \mathrm{~mL})$ and extracted with DCM $(5 \times 8 \mathrm{~mL})$. The combined organic layers were washed twice with water, once with brine, dried over $\mathrm{MgSO}_{4}$, filtered and concentrated. ISCO flash chromatography (12 g silica, 0-10\% MeOH/DCM, 12 min gradient) provided the title compound as a yellow semi-solid (64.9 mg, 25\% yield).

${ }^{1} \mathrm{H}$ NMR $\left(500 \mathrm{MHz}, \mathrm{CDCl}_{3}\right) \delta 8.09(\mathrm{~s}, 1 \mathrm{H}), 3.93(\mathrm{t}, \mathrm{J}=7.2 \mathrm{~Hz}, 2 \mathrm{H}), 2.62(\mathrm{~s}, 3 \mathrm{H}), 1.84(\mathrm{~h}, \mathrm{~J}=7.4$ $\mathrm{Hz}, 2 \mathrm{H}), 1.00(\mathrm{t}, \mathrm{J}=7.4 \mathrm{~Hz}, 3 \mathrm{H})$. LRMS (ESI) calculated for $\mathrm{C}_{13} \mathrm{H}_{15} \mathrm{ClN}_{5} \mathrm{OS}[\mathrm{M}+\mathrm{H}]^{+} 324.07$, found 323.77 .

Synthesis of 2-methylthiazole-4-N-trifluoroethyl core
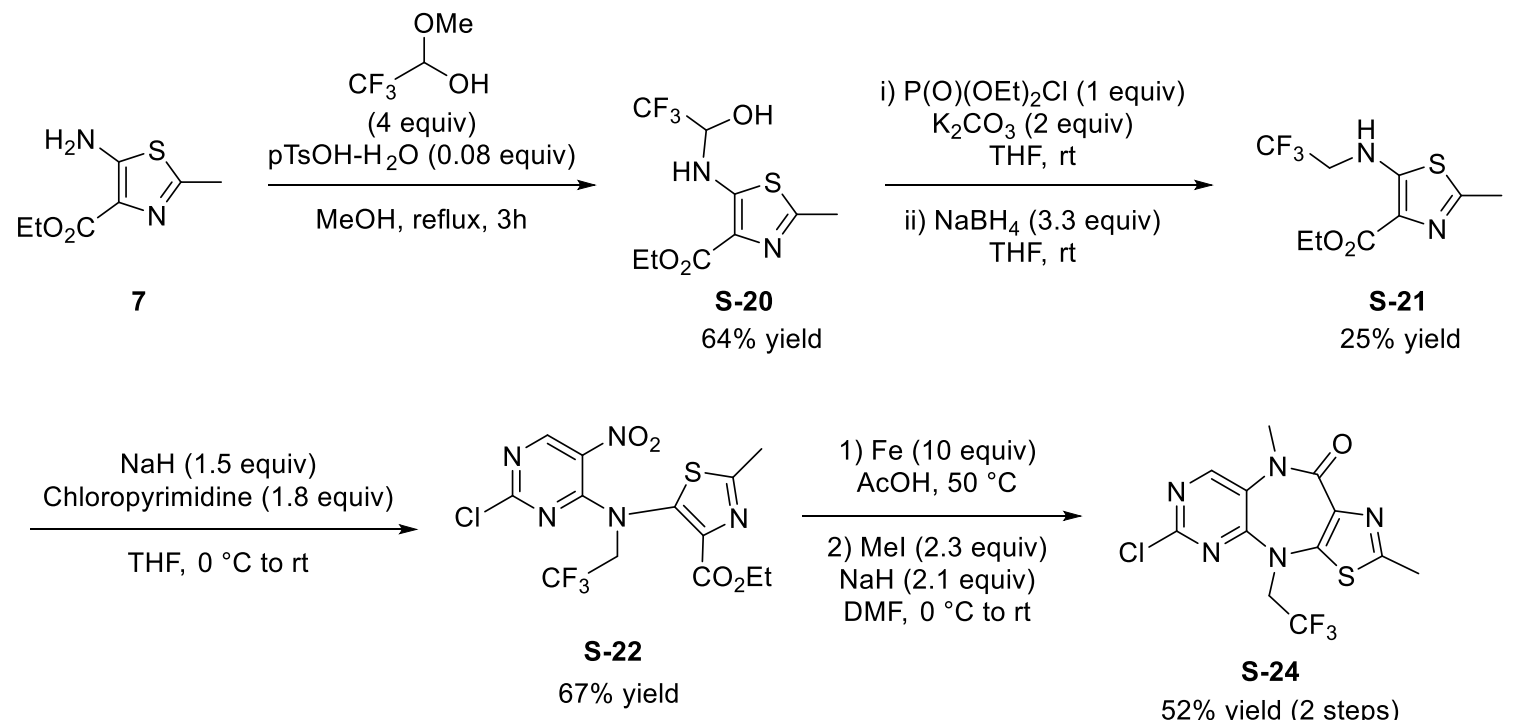

Ethyl 2-methyl-5-((2,2,2-trifluoro-1-hydroxyethyl)amino)thiazole-4-carboxylate (S-20 $)^{11}$<smiles>CCOC(=O)c1nc(C)sc1NC(O)C(F)(F)F</smiles> 
To a suspension of ethyl 5-amino-2-methylthiazole-4-carboxylate 7 ( $701 \mathrm{mg}, 3.5 \mathrm{mmol}, 1.0$ equiv) and p-toluenesulfonic acid monohydrate $(52.5 \mathrm{mg}, 0.28 \mathrm{mmol}, 0.08$ equiv) in methanol $(9 \mathrm{~mL})$, trifluoroacetaldehyde methyl hemiacetal $(1.34 \mathrm{~mL}, 14.0 \mathrm{mmol}, 4.0$ equiv) was added and the mixture was heated to $70{ }^{\circ} \mathrm{C}$ for three hours. The reaction was then cooled, diluted with EtOAc $(30 \mathrm{~mL})$ and poured into $100 \mathrm{~mL}$ saturated aqueous $\mathrm{NaHCO}_{3}$. The organic layer was separated, and the aqueous component was extracted with EtOAc $(2 \times 30 \mathrm{~mL})$. The combined organic layers were washed twice with water and once with brine, dried over $\mathrm{MgSO}_{4}$, filtered and concentrated. ISCO flash chromatography ( $15-70 \%$ EtOAC/hex, 20 minutes, $40 \mathrm{~g}$ silica) provided the title compound as a viscous yellow oil (644 $\mathrm{mg}, 64 \%$ isolated yield).

${ }^{1} \mathrm{H}$ NMR (500 MHz, CDCl $) \delta 7.88(\mathrm{~d}, \mathrm{~J}=8.7 \mathrm{~Hz}, 1 \mathrm{H}), 5.08(\mathrm{dq}, \mathrm{J}=8.7,4.3 \mathrm{~Hz}, 1 \mathrm{H}), 4.75(\mathrm{~d}, \mathrm{~J}=$ $3.7 \mathrm{~Hz}, 1 \mathrm{H}), 4.39$ (q, J = 7.1 Hz, 2H), 2.56 (s, 3H), 1.39 (t, J = 7.1 Hz, 3H). ${ }^{19} \mathrm{~F}$ NMR (471 MHz, $\left.\mathrm{CDCl}_{3}\right) \delta-82.02\left(\mathrm{~d}, \mathrm{~J}=4.5 \mathrm{~Hz}\right.$ ). LRMS (ESI) calculated for $\mathrm{C}_{9} \mathrm{H}_{12} \mathrm{~F}_{3} \mathrm{~N}_{2} \mathrm{O}_{3} \mathrm{~S}[\mathrm{M}+\mathrm{H}]^{+} 285.05$, found 284.77 .

Ethyl 2-methyl-5-((2,2,2-trifluoroethyl)amino)thiazole-4-carboxylate (S-21)

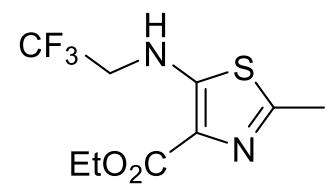

To a suspension of ethyl 2-methyl-5-((2,2,2-trifluoro-1-hydroxyethyl)amino)thiazole-4carboxylate $\mathbf{S - 2 0}$ (810 mg, $2.8 \mathrm{mmol}, 1.0$ equiv) and $\mathrm{K}_{2} \mathrm{CO}_{3}(781 \mathrm{mg}, 5.6 \mathrm{mmol}, 2.0$ equiv) in anhydrous THF (15 mL), diethyl chlorophosphate $(0.42 \mathrm{~mL}, 2.8 \mathrm{mmol}, 1.0$ equiv) was added at room temperature and the reaction was for 14 hours. The mixture was then diluted with anhydrous THF ( $8 \mathrm{~mL}$ ) and $\mathrm{NaBH}_{4}$ (346 mg, $9.1 \mathrm{mmol}, 3.3$ equiv) was added. The reaction was stirred for 6 hours, and then quenched with dilute aqueous $\mathrm{NH}_{4} \mathrm{Cl}$. The aqueous component was extracted with EtOAc $(3 \times 25 \mathrm{~mL})$. The combined organic layers were washed with water and brine, dried over $\mathrm{MgSO}_{4}$, filtered and concentrated. ISCO flash chromatography (15 - 70\% EtOAC/hex, 20 minutes, $24 \mathrm{~g}$ silica) provided the title compound as an off-white solid (189.8 $\mathrm{mg}, 25 \%$ yield). 
${ }^{1} \mathrm{H}$ NMR $\left(500 \mathrm{MHz}, \mathrm{CDCl}_{3}\right) \delta 7.66(\mathrm{t}, \mathrm{J}=6.1 \mathrm{~Hz}, 1 \mathrm{H}), 4.40(\mathrm{q}, \mathrm{J}=7.1 \mathrm{~Hz}, 2 \mathrm{H}), 3.80(\mathrm{qd}, \mathrm{J}=8.5$, $7.0 \mathrm{~Hz}, 2 \mathrm{H}), 2.58(\mathrm{~s}, 3 \mathrm{H}), 1.41(\mathrm{t}, \mathrm{J}=7.1 \mathrm{~Hz}, 3 \mathrm{H}) .{ }^{19} \mathrm{~F} \mathrm{NMR}\left(471 \mathrm{MHz}, \mathrm{CDCl}_{3}\right) \delta-72.46(\mathrm{t}, \mathrm{J}=$ 8.5 Hz). LRMS (ESI) calculated for $\mathrm{C}_{9} \mathrm{H}_{12} \mathrm{~F}_{3} \mathrm{~N}_{2} \mathrm{O}_{2} \mathrm{~S}[\mathrm{M}+\mathrm{H}]^{+}$269.06, found 268.87.

\section{Ethyl 5-((2-chloro-5-nitropyrimidin-4-yl)(2,2,2-trifluoroethyl)amino)-2-methylthiazole-4-} carboxylate ( $\mathrm{S}-22)$<smiles>CCOC(=O)c1nc(C)sc1N(CC(F)(F)F)c1nc(Cl)ncc1[N+](=O)[O-]</smiles>

General Procedure B was followed on $0.70 \mathrm{mmol}$ scale using ethyl 2-methyl-5-((2,2,2trifluoroethyl)amino)thiazole-4-carboxylate S-21 (186.2 $\mathrm{mg}$ ) and 2,4-dichloro-5-nitropyrimidine (245 mg, 1.8 equiv) as the electrophile. The reaction was run at $60{ }^{\circ} \mathrm{C}$ overnight. ISCO flash chromatography $(15-60 \%$ EtOAC/hex, 16 minutes, $12 \mathrm{~g}$ silica) provided the title compound as a yellow oil (199.9 mg, 67\% isolated yield).

${ }^{1} \mathrm{H}$ NMR (500 MHz, $\left.\mathrm{CDCl}_{3}\right) \delta 8.63(\mathrm{~s}, 1 \mathrm{H}), 4.77(\mathrm{~s}, 2 \mathrm{H}), 4.27(\mathrm{q}, \mathrm{J}=7.1 \mathrm{~Hz}, 2 \mathrm{H}), 2.74(\mathrm{~s}, 3 \mathrm{H})$, $1.26(\mathrm{t}, \mathrm{J}=7.1 \mathrm{~Hz}, 3 \mathrm{H}) .{ }^{19} \mathrm{~F}$ NMR $\left(471 \mathrm{MHz}, \mathrm{CDCl}_{3}\right) \delta-68.21(\mathrm{t}, \mathrm{J}=8.4 \mathrm{~Hz})$. LRMS (ESI) calculated for $\mathrm{C}_{13} \mathrm{H}_{12} \mathrm{ClF}_{3} \mathrm{~N}_{5} \mathrm{O}_{4} \mathrm{~S}[\mathrm{M}+\mathrm{H}]^{+} 426.02$, found 426.29 .

\section{6-chloro-2-methyl-4-(2,2,2-trifluoroethyl)-4,9-dihydro-10H-pyrimido[5,4-b]thiazolo[5,4-} e][1,4]diazepin-10-one (S-23)<smiles>Cc1nc2c(s1)N(CC(F)(F)F)c1nc(Cl)ncc1NC2=O</smiles>

General Procedure C was followed on $0.48 \mathrm{mmol}$ scale using ethyl 5-((2-chloro-5-nitropyrimidin4-yl)(2,2,2-trifluoroethyl)amino)-2-methylthiazole-4-carboxylate $\mathbf{S - 2 2}$ (195.9 mg) for 18 hours. Modified isolation: after removing iron, the crude reaction mixture was poured into $50 \mathrm{~mL}$ water and extracted with DCM $(4 \times 10 \mathrm{~mL})$. The combined organic layers were washed twice with sat. 
aqueous $\mathrm{NaHCO}_{3}$ and then brine, dried over $\mathrm{MgSO}_{4}$, filtered and concentrated to provide the title compound as a yellow foam $(154.0 \mathrm{mg})$.

${ }^{1} \mathrm{H}$ NMR (500 MHz, DMSO-d6) $\delta 10.27(\mathrm{~s}, 1 \mathrm{H}), 8.34(\mathrm{~s}, 1 \mathrm{H}), 4.96(\mathrm{q}, \mathrm{J}=8.8 \mathrm{~Hz}, 2 \mathrm{H}), 2.57$ (s, $3 \mathrm{H}) .{ }^{19} \mathrm{~F}$ NMR (471 MHz, DMSO-d6) $\delta-69.58(\mathrm{t}, \mathrm{J}=8.9 \mathrm{~Hz})$. LRMS (ESI) calculated for $\mathrm{C}_{11} \mathrm{H}_{8} \mathrm{ClF}_{3} \mathrm{~N}_{5} \mathrm{OS}[\mathrm{M}+\mathrm{H}]^{+} 350.01$, found 349.77 .

6-chloro-2,9-dimethyl-4-(2,2,2-trifluoroethyl)-4,9-dihydro-10H-pyrimido[5,4-b]thiazolo[5,4e][1,4]diazepin-10-one (S-24)<smiles>Cc1nc2c(s1)N(CC(F)(F)F)c1nc(Cl)ncc1N(C)C2=O</smiles>

General Procedure D was followed on $0.35 \mathrm{mmol}$ scale using $\mathbf{S - 2 3}(122.8 \mathrm{mg})$ and iodomethane ( $50.0 \mu \mathrm{L}, 0.80 \mathrm{mmol}, 2.3$ equiv) as the electrophile, for 2 hours. The reaction was then quenched with water $(20 \mathrm{~mL})$ and extracted with DCM $(4 \times 10 \mathrm{~mL})$. The combined organic layers were washed twice with water, once with brine, dried over $\mathrm{MgSO}_{4}$, filtered and concentrated. ISCO flash chromatography ( $12 \mathrm{~g}$ silica, $0-10 \% \mathrm{MeOH} / \mathrm{DCM}, 12 \mathrm{~min}$ gradient) provided the title compound as a yellow oil (91.1 $\mathrm{mg}, 52 \%$ yield over 2 steps).

${ }^{1} \mathrm{H}$ NMR (500 MHz, $\left.\mathrm{CDCl}_{3}\right) \delta 8.39(\mathrm{~s}, 1 \mathrm{H}), 4.69(\mathrm{q}, \mathrm{J}=8.1 \mathrm{~Hz}, 2 \mathrm{H}), 3.47(\mathrm{~s}, 3 \mathrm{H}), 2.62(\mathrm{~s}, 3 \mathrm{H}) .{ }^{19} \mathrm{~F}$ NMR (471 MHz, $\left.\mathrm{CDCl}_{3}\right) \delta-70.87\left(\mathrm{t}, \mathrm{J}=8.1 \mathrm{~Hz}\right.$ ). LRMS (ESI) calculated for $\mathrm{C}_{12} \mathrm{H}_{10} \mathrm{ClF}_{3} \mathrm{~N}_{5} \mathrm{OS}$ $[\mathrm{M}+\mathrm{H}]^{+} 364.02$, found 363.77 . 

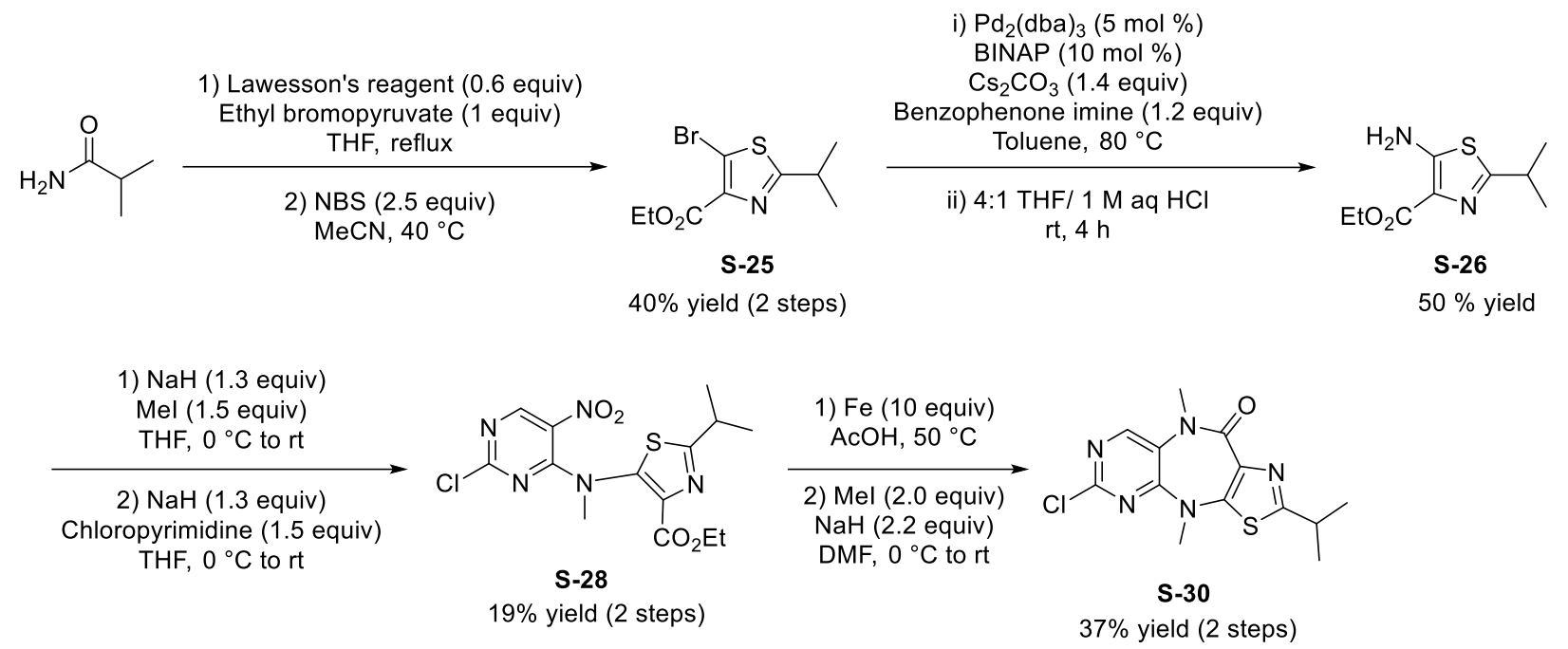

Ethyl 5-bromo-2-isopropylthiazole-4-carboxylate (S-25) ${ }^{12}$<smiles>CCOC(=O)c1nc(C(C)C)sc1Br</smiles>

A suspension of isobutyramide ( $2.62 \mathrm{~g}, 30.0 \mathrm{mmol}, 1.0$ equiv) and Lawesson's reagent (7.23 g, $18.0 \mathrm{mmol}, 0.6$ equiv) in anhydrous THF $(40 \mathrm{~mL})$ was heated to $70^{\circ} \mathrm{C}$ for 24 hours. The reaction flask was removed from the heat, cooled to room temperature, and ethyl bromopyruvate $(3.75 \mathrm{~mL}$, $30.0 \mathrm{mmol}, 1.0$ equiv) was added, and then the reaction was returned to $70{ }^{\circ} \mathrm{C}$ for 2 days. The reaction mixture was then cooled to room temperature, quenched with water $(100 \mathrm{~mL})$, and extracted with EtOAc $(3 \times 75 \mathrm{~mL})$. The combined organic layers were washed with brine, dried over $\mathrm{MgSO}_{4}$, filtered and concentrated. ISCO flash chromatography (80 g silica, 10-60\% EtOAc/Hex, 28 min gradient) provided ethyl 2-isopropylthiazole-4-carboxylate as a yellow oil (7.3 g, quantitative yield, $\sim 50 \%$ purity).

${ }^{1} \mathrm{H}$ NMR $\left(500 \mathrm{MHz}, \mathrm{CDCl}_{3}\right) \delta 8.06(\mathrm{~s}, 1 \mathrm{H}), 4.42(\mathrm{q}, \mathrm{J}=7.1 \mathrm{~Hz}, 2 \mathrm{H}), 3.54-3.47(\mathrm{~m}, 1 \mathrm{H}), 1.45-$ $1.38(\mathrm{~m}, 9 \mathrm{H})$. MS (ESI) $\mathrm{m} / \mathrm{z}: 200.08(\mathrm{M}+\mathrm{H})^{+}$.

To the impure mixture of ethyl 2-isopropylthiazole-4-carboxylate ( $7.3 \mathrm{~g}, 18.3 \mathrm{mmol}, 1$ equiv) in acetonitrile $(37 \mathrm{~mL})$ was added $\mathrm{N}$-bromosuccinimide $(8.14 \mathrm{~g}, 45.8 \mathrm{mmol}, 2.5$ equiv). The mixture 
was heated to $40{ }^{\circ} \mathrm{C}$ for 24 hours. The reaction was then diluted with DCM (100 mL) and water $(200 \mathrm{~mL})$. The organic layer was removed, and the aqueous layer extracted with DCM $(3 \times 75 \mathrm{~mL})$. The combined organic layers were washed twice with water, once with brine, dried over $\mathrm{MgSO}_{4}$, filtered and then concentrated to provide a dark, viscous oil. ISCO flash chromatography (40 g silica, 15-80\% EtOAc/hexanes, 11 min gradient) provided the title compound as a light yellow solid (3.35 g, 40\% yield over 2 steps).

LRMS (ESI) calculated for $\mathrm{C}_{9} \mathrm{H}_{13} \mathrm{BrNO}_{2} \mathrm{~S}[\mathrm{M}+\mathrm{H}]^{+}$277.98, found 277.77, 279.77 .

\section{Ethyl 5-amino-2-isopropylthiazole-4-carboxylate (S-26)}<smiles>CCOC(=O)c1nc(C(C)C)sc1N</smiles>

A mixture of ethyl 5-bromo-2-isopropylthiazole-4-carboxylate $\mathbf{S - 2 5}$ (1.6 g, 5.75 mmol, 1.0 equiv), $\mathrm{Pd}_{2}(\mathrm{dba})_{3}(262 \mathrm{mg}, 0.29 \mathrm{mmol}, 0.05$ equiv), ( \pm )-BINAP (358 mg, $0.58 \mathrm{mmol}, 0.10$ equiv), cesium carbonate $(2.61 \mathrm{~g}, 8.0 \mathrm{mmol}, 1.4$ equiv), and benzophenone imine (1.16 mL, $6.9 \mathrm{mmol}, 1.2$ equiv) in anhydrous toluene ( $23 \mathrm{~mL}$ ) was sparged with $\mathrm{N}_{2}$ for 5 minutes. The reaction was heated to 80 ${ }^{\circ} \mathrm{C}$ overnight, and then cooled to room temperature, diluted with EtOAc (100 mL) and filtered through Celite. Solvents were removed in vacuo to provide a dark red syrup. The intermediate ethyl 5-((diphenylmethylene)amino)-2-isopropylthiazole-4-carboxylate was carried on directly to hydrolysis.

LRMS (ESI) calculated for $\mathrm{C}_{22} \mathrm{H}_{23} \mathrm{~N}_{2} \mathrm{O}_{2} \mathrm{~S}[\mathrm{M}+\mathrm{H}]^{+} 379.15$, found 378.97 .

Crude ethyl 5-((diphenylmethylene)amino)-2-isopropylthiazole-4-carboxylate was dissolved in 20 $\mathrm{mL}$ THF and $4 \mathrm{~mL} 1 \mathrm{M} \mathrm{HCl}$. The mixture was stirred at room temperature overnight, at which point UPLC-MS analysis showed complete hydrolysis of the imine. The reaction was diluted with $100 \mathrm{~mL}$ EtOAc, neutralized to $\mathrm{pH} \sim 10$, washed twice with water and once with brine, dried over $\mathrm{MgSO}_{4}$, filtered and concentrated. ISCO flash chromatography (24 g silica, 25-80\% EtOAc/hexanes, $10 \mathrm{~min}$ gradient) to provide the title compound as a yellow-orange solid (660 mg, $50 \%$ yield). 
${ }^{1} \mathrm{H}$ NMR (500 MHz, CDCl $) \delta 5.87$ (s, 2H), 4.39 (q, J = 7.1 Hz, 2H), 3.25 (h, J = 7.0 Hz, 1H), 1.40 $(\mathrm{t}, \mathrm{J}=7.1 \mathrm{~Hz}, 3 \mathrm{H}), 1.32(\mathrm{~d}, \mathrm{~J}=6.9 \mathrm{~Hz}, 6 \mathrm{H})$. LRMS (ESI) calculated for $\mathrm{C}_{9} \mathrm{H}_{15} \mathrm{~N}_{2} \mathrm{O}_{2} \mathrm{~S}[\mathrm{M}+\mathrm{H}]^{+}$ 215.08, found 215.08.

Ethyl 2-isopropyl-5-(methylamino)thiazole-4-carboxylate (S-27)<smiles>CCOC(=O)c1nc(C(C)C)sc1NC</smiles>

General Procedure B was followed on $3.4 \mathrm{mmol}$ scale using ethyl 5-amino-2-phenylthiazole-4carboxylate $\mathbf{S - 2 6}(728 \mathrm{mg})$ and iodomethane $(0.31 \mathrm{~mL}, 5.1 \mathrm{mmol}, 1.5$ equiv) as the electrophile. ISCO flash chromatography (20-80\% EtOAC/hex, 10 minutes, 12 g silica) provided the title compound as a yellow solid (205 mg, 26\% yield).

LRMS (ESI) calculated for $\mathrm{C}_{10} \mathrm{H}_{17} \mathrm{~N}_{2} \mathrm{O}_{2} \mathrm{~S}[\mathrm{M}+\mathrm{H}]^{+} 229.10$, found 229.08.

Ethyl 5-((2-chloro-5-nitropyrimidin-4-yl)(methyl)amino)-2-isopropylthiazole-4-carboxylate (S-28)<smiles>CCOC(=O)c1nc(C(C)C)sc1N(C)c1nc(Cl)ncc1[N+](=O)[O-]</smiles>

General Procedure B was followed on $1.2 \mathrm{mmol}$ scale using ethyl 2-isopropyl-5(methylamino)thiazole-4-carboxylate (256 mg) and 2,4-dichloro-5-nitropyrimidine (350 mg, 1.8 mmol, 1.5 equiv) as the electrophile. ISCO flash chromatography (20-80\% EtOAC/hex, 15 minutes, $40 \mathrm{~g}$ silica) provided the title compound as a red-orange oil (350 $\mathrm{mg}$, $75 \%$ isolated yield). LRMS (ESI) calculated for $\mathrm{C}_{14} \mathrm{H}_{17} \mathrm{ClN}_{5} \mathrm{O}_{4} \mathrm{~S}[\mathrm{M}+\mathrm{H}]^{+} 386.07$, found 385.87 . 
<smiles>CC(C)c1nc2c(s1)N(C)c1nc(Cl)ncc1NC2=O</smiles>

General Procedure C was followed on 0.9 mmol scale using S-28 (350 mg). The crude reaction mixture was poured into $100 \mathrm{~mL}$ water and stirred for 30 minutes. The precipitate was collected by suction filtration, washing with $50 \mathrm{~mL}$ water to provide the title compound as a yellow solid (110 mg, 39\% yield).

${ }^{1} \mathrm{H}$ NMR (500 MHz, DMSO-d6) $\delta 9.95$ (s, 1H), 8.11 (s, 1H), 3.37 (s, 3H), 3.16 (p, J = 6.9 Hz, 1H), $1.28(\mathrm{~d}, \mathrm{~J}=6.9 \mathrm{~Hz}, 6 \mathrm{H})$. LRMS (ESI) calculated for $\mathrm{C}_{12} \mathrm{H}_{13} \mathrm{ClN}_{5} \mathrm{OS}[\mathrm{M}+\mathrm{H}]^{+} 310.05$, found 309.77 .

\section{6-chloro-2-isopropyl-4,9-dimethyl-4,9-dihydro-10H-pyrimido[5,4-b]thiazolo[5,4-} e][1,4]diazepin-10-one (S-30)<smiles>CC(C)c1nc2c(s1)N(C)c1nc(Cl)ncc1N(C)C2=O</smiles>

To a suspension of 6-chloro-2-isopropyl-4-methyl-4,9-dihydro-10H-pyrimido[5,4-b]thiazolo[5,4e][1,4]diazepin-10-one $\mathbf{S - 2 9}$ (110 $\mathrm{mg}, 0.36 \mathrm{mmol}, 1.0$ equiv) and iodomethane $(50.0 \mu \mathrm{L}, 0.80$ mmol, 2.2 equiv) in anhydrous DMF $(3.5 \mathrm{~mL})$ at $0{ }^{\circ} \mathrm{C}, \mathrm{NaH}(18.8 \mathrm{mg}, 0.78 \mathrm{mmol}, 2.1$ equiv) was added in a single portion. The reaction was stirred overnight and then quenched with water (20 $\mathrm{mL})$ and extracted with EtOAc $(5 \times 10 \mathrm{~mL})$. The combined organic layers were washed twice with water, once with brine, dried over $\mathrm{MgSO}_{4}$, filtered and concentrated to provide the title compound as a yellow oil (110 mg, 95\% yield). The material was sufficiently pure by NMR analysis and not purified further. 
${ }^{1} \mathrm{H}$ NMR (500 MHz, DMSO-d6) $\delta 8.57$ (s, 1H), 3.38 (s, 3H), 3.31 (s, 3H), 3.17 (p, J = 6.9 Hz, 1H), $1.27(\mathrm{~d}, \mathrm{~J}=6.9 \mathrm{~Hz}, 6 \mathrm{H})$. LRMS (ESI) calculated for $\mathrm{C}_{13} \mathrm{H}_{15} \mathrm{ClN}_{5} \mathrm{OS}[\mathrm{M}+\mathrm{H}]^{+}$324.07, found 323.87 .

Synthesis of 2-phenylthiazole core

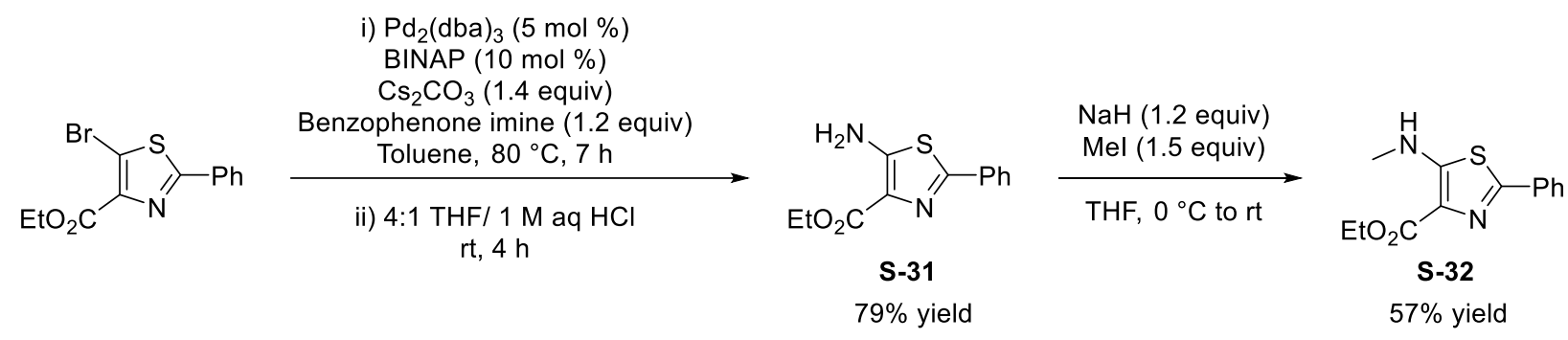

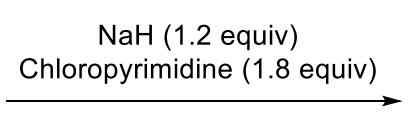

THF, $0{ }^{\circ} \mathrm{C}$ to $\mathrm{rt}$

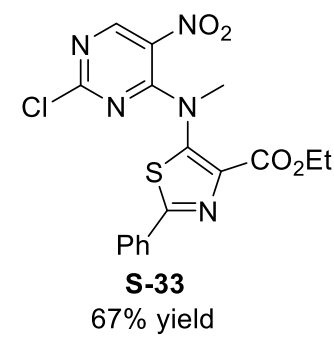

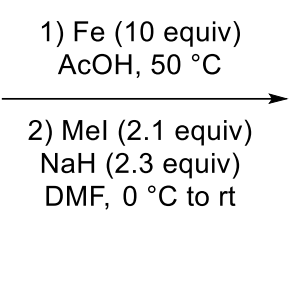

$\mathrm{DMF}, 0^{\circ} \mathrm{C}$ to rt

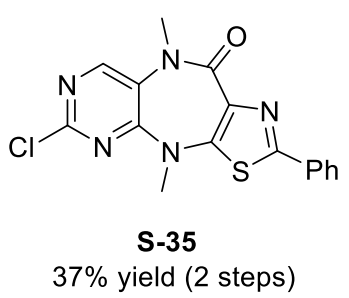

\section{Ethyl 5-amino-2-phenylthiazole-4-carboxylate (S-31)}<smiles>CCOC(=O)c1nc(-c2ccccc2)sc1N</smiles>

A mixture of ethyl 5-bromo-2-phenylthiazole-4-carboxylate (2.015 g, $6.5 \mathrm{mmol}, 1.0$ equiv), $\mathrm{Pd}_{2}(\mathrm{dba})_{3}$ (298 mg, $0.33 \mathrm{mmol}, 0.05$ equiv), ( \pm )-BINAP (405 mg, $0.65 \mathrm{mmol}, 0.10$ equiv), and cesium carbonate $(2.965 \mathrm{~g}, 9.1 \mathrm{mmol}, 1.4$ equiv), and benzophenone imine $(1.31 \mathrm{~mL}, 7.8 \mathrm{mmol}$, 1.2 equiv) in anhydrous toluene $(25 \mathrm{~mL})$ was sparged with $\mathrm{N}_{2}$ for 10 minutes. The reaction was heated to $80^{\circ} \mathrm{C}$ for 19 hours, and then cooled to room temperature, diluted with EtOAc (100 mL) and filtered through Celite. Solvents were removed in vacuo to provide a dark red syrup. The intermediate ethyl 5-((diphenylmethylene)amino)-2-phenylthiazole-4-carboxylate was carried on directly to hydrolysis.

LRMS (ESI) calculated for $\mathrm{C}_{25} \mathrm{H}_{21} \mathrm{~N}_{2} \mathrm{O}_{2} \mathrm{~S}[\mathrm{M}+\mathrm{H}]^{+}$413.13, found 412.87. 
Crude ethyl 5-((diphenylmethylene)amino)-2-phenylthiazole-4-carboxylate was dissolved in 25 $\mathrm{mL}$ THF and $8 \mathrm{~mL} 1 \mathrm{M} \mathrm{HCl}$. The mixture was stirred at room temperature for 5 hours, at which point UPLC-MS analysis showed complete hydrolysis of the imine. The reaction was diluted with $100 \mathrm{~mL}$ EtOAc, neutralized to $\mathrm{pH} \sim 10$, washed twice with water and once with brine, dried over $\mathrm{MgSO}_{4}$, filtered and concentrated. ISCO flash chromatography (40 g silica, 25-80\% EtOAc/Hex, 20 min gradient) to provide the title compound as a yellow solid (1.273 g, 79\% yield over 2 steps).

${ }^{1} \mathrm{H} \mathrm{NMR}\left(500 \mathrm{MHz}, \mathrm{CDCl}_{3}\right) \delta 7.82-7.76(\mathrm{~m}, 2 \mathrm{H}), 7.43-7.33(\mathrm{~m}, 3 \mathrm{H}), 5.89$ (br s, 2H), 4.43 (q, $J=7.1 \mathrm{~Hz}, 2 \mathrm{H}), 1.44(\mathrm{t}, J=7.1 \mathrm{~Hz}, 3 \mathrm{H})$. LRMS (ESI) calculated for $\mathrm{C}_{12} \mathrm{H}_{13} \mathrm{~N}_{2} \mathrm{O}_{2} \mathrm{~S}[\mathrm{M}+\mathrm{H}]^{+} 249.07$, found 248.88 .

\section{Ethyl 5-(methylamino)-2-phenylthiazole-4-carboxylate (S-32)}<smiles>CCOC(=O)c1nc(-c2ccccc2)sc1NC</smiles>

General Procedure B was followed on $4.0 \mathrm{mmol}$ scale using ethyl 5-amino-2-phenylthiazole-4carboxylate S-31 (1.018 g) and iodomethane (0.37 mL, 1.5 equiv) as the electrophile, with a reaction time of 3 hours. ISCO flash chromatography (25-80\% EtOAc/hex, 19 minutes, $40 \mathrm{~g}$ silica) provided the title compound as a yellow viscous oil (620 mg, $57 \%$ yield).

${ }^{1} \mathrm{H}$ NMR $\left(500 \mathrm{MHz}, \mathrm{CDCl}_{3}\right) \delta 7.87-7.75(\mathrm{~m}, 2 \mathrm{H}), 7.43-7.34(\mathrm{~m}, 3 \mathrm{H}), 6.08(\mathrm{~s}, 1 \mathrm{H}), 4.41(\mathrm{t}, J=$ $7.1 \mathrm{~Hz}, 2 \mathrm{H}), 3.11(\mathrm{~s}, 3 \mathrm{H}), 1.43(\mathrm{t}, J=7.1 \mathrm{~Hz}, 3 \mathrm{H})$. LRMS (ESI) calculated for $\mathrm{C}_{13} \mathrm{H}_{15} \mathrm{~N}_{2} \mathrm{O}_{2} \mathrm{~S}$ $[\mathrm{M}+\mathrm{H}]^{+}$263.08, found 262.87 .

Ethyl 5-((2-chloro-5-nitropyrimidin-4-yl)(methyl)amino)-2-phenylthiazole-4-carboxylate (S33)<smiles>CCOC(=O)c1nc(-c2ccccc2)sc1N(C)c1nc(Cl)ncc1[N+](=O)[O-]</smiles> 
General Procedure B was followed on $2.3 \mathrm{mmol}$ scale, using ethyl 5-(methylamino)-2phenylthiazole-4-carboxylate S-32 (620 mg) and 2,4-dichloro-5-nitropyrimidine (796 mg, 4.14 mmol, 1.8 equiv) as the electrophile. The reaction was stirred at room temperature overnight. ISCO flash chromatography (20-70\% EtOAc/hex, 18 minutes, $24 \mathrm{~g}$ silica) provided the title compound as a yellow-orange foam (655 $\mathrm{mg}, 67 \%$ isolated yield).

${ }^{1} \mathrm{H}$ NMR $\left(500 \mathrm{MHz}, \mathrm{CDCl}_{3}\right) \delta 8.62(\mathrm{~s}, 1 \mathrm{H}), 7.97-7.90(\mathrm{~m}, 2 \mathrm{H}), 7.52-7.44(\mathrm{~m}, 3 \mathrm{H}), 4.28(\mathrm{q}, J=$ $7.1 \mathrm{~Hz}, 2 \mathrm{H}), 3.69(\mathrm{~s}, 3 \mathrm{H}), 1.28\left(\mathrm{t}, J=7.1 \mathrm{~Hz}, 3 \mathrm{H}\right.$ ). LRMS (ESI) calculated for $\mathrm{C}_{17} \mathrm{H}_{15} \mathrm{ClN}_{5} \mathrm{O}_{4} \mathrm{~S}$ $[\mathrm{M}+\mathrm{H}]^{+} 420.05$, found 419.77 .

\section{6-chloro-4-methyl-2-phenyl-4,9-dihydro-10H-pyrimido[5,4-b]thiazolo[5,4-e][1,4]diazepin-} 10-one (S-34)<smiles>CN1c2nc(Cl)ncc2NC(=O)c2nc(-c3ccccc3)sc21</smiles>

General Procedure $\mathrm{C}$ was followed on $1.50 \mathrm{mmol}$ scale, using $\mathbf{S}-33(655 \mathrm{mg})$ at $50{ }^{\circ} \mathrm{C}$ for 16 hours. The title compound was isolated by suction filtration as a yellow solid ( $321 \mathrm{mg}, 60 \%$ yield).

${ }^{1} \mathrm{H}$ NMR (500 MHz, DMSO-d6) $\delta 10.09$ (s, 1H), 8.16 (s, 1H), 7.89 - 7.77 (m, 2H), 7.64 - 7.35 (m, 3H), $3.46(\mathrm{~s}, 3 \mathrm{H})$. MS (ESI) calculated for $\mathrm{C}_{15} \mathrm{H}_{11} \mathrm{ClN}_{5} \mathrm{OS}[\mathrm{M}+\mathrm{H}]^{+} 344.04$, found 343.77 .

\section{6-chloro-4,9-dimethyl-2-phenyl-4,9-dihydro-10H-pyrimido[5,4-b]thiazolo[5,4- e][1,4]diazepin-10-one (S-35)}<smiles>CN1C(=O)c2nc(-c3ccccc3)sc2N(C)c2nc(Cl)ncc21</smiles>

General Procedure D was followed on $0.70 \mathrm{mmol}$ scale, using S-34 (246 mg) and iodomethane ( $0.090 \mathrm{~mL}, 1.40 \mathrm{mmol}, 2.0$ equiv) as the electrophile, with a reaction time of 2 hours. The reaction was then quenched with water $(20 \mathrm{~mL})$ and extracted with DCM $(5 \times 10 \mathrm{~mL})$. The combined organic 
layers were washed three times with water, once with brine, dried over $\mathrm{MgSO}_{4}$, filtered and concentrated. ISCO flash chromatography (12 g silica, 0-10\% MeOH/DCM, 12 min gradient) provided the title compound as a red-orange solid (156.4 mg, 62\% yield).

${ }^{1} \mathrm{H}$ NMR (500 MHz, DMSO-d6) $\delta 8.63(\mathrm{~s}, 1 \mathrm{H}), 7.88$ - $7.81(\mathrm{~m}, 2 \mathrm{H}), 7.53$ - $7.44(\mathrm{~m}, 3 \mathrm{H}), 3.47$ (s, $3 \mathrm{H}), 3.35$ (s, 3H). LRMS (ESI) calculated for $\mathrm{C}_{16} \mathrm{H}_{13} \mathrm{ClN}_{5} \mathrm{OS}[\mathrm{M}+\mathrm{H}]^{+} 358.05$, found 357.77 .

Synthesis of 2-trifluoromethylthiazole core
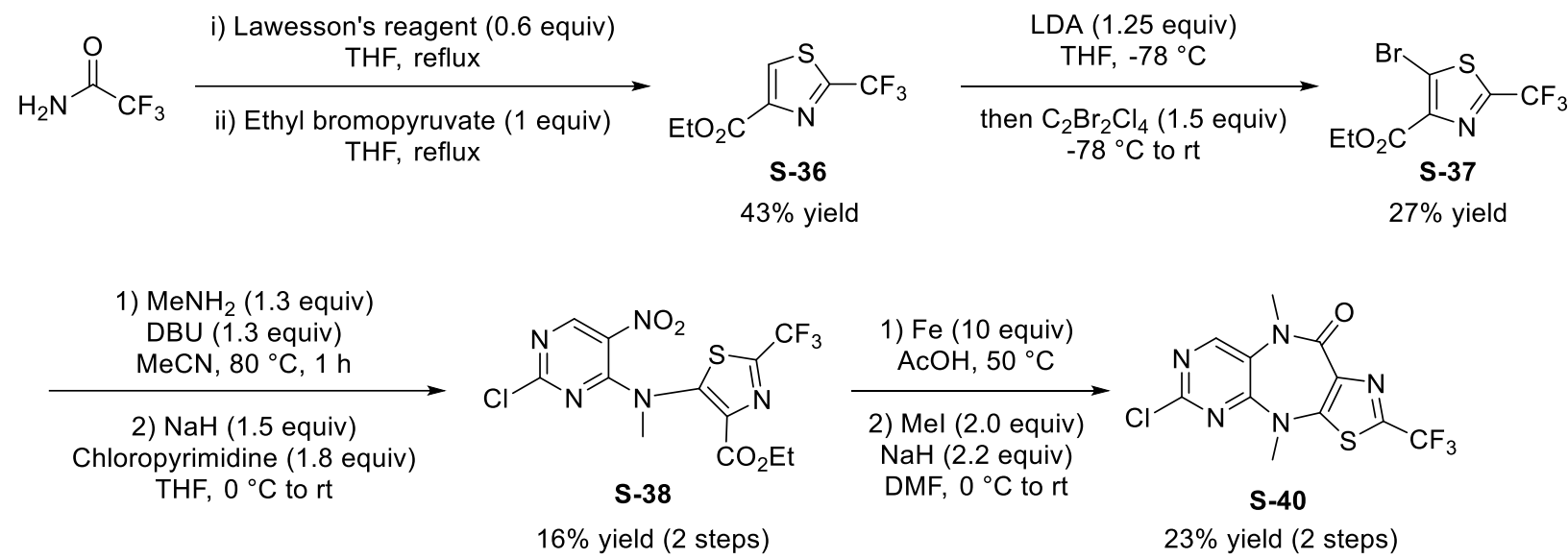

Ethyl 2-(trifluoromethyl)thiazole-4-carboxylate (S-36)<smiles>CCOC(OCC)c1csc(C(F)(F)F)n1</smiles>

A suspension of trifluoroacetamide $(2.267 \mathrm{~g}, 20.0 \mathrm{mmol}, 1.0$ equiv) and Lawesson's reagent (4.835 $\mathrm{g}, 12.0 \mathrm{mmol}, 0.6$ equiv) in anhydrous THF $(40 \mathrm{~mL})$ was heated to $70{ }^{\circ} \mathrm{C}$ for 2 days. The reaction flask was removed from the heat, cooled to room temperature, and ethyl bromopyruvate $(2.60 \mathrm{~mL}$, $20.0 \mathrm{mmol}, 1.0$ equiv) was added, and then the reaction was returned to $70{ }^{\circ} \mathrm{C}$ for 18 hours. The reaction mixture was then cooled to room temperature, quenched with water $(100 \mathrm{~mL})$, and extracted with EtOAc $(3 \times 75 \mathrm{~mL})$. The combined organic layers were washed with brine, dried over $\mathrm{MgSO}_{4}$, filtered and concentrated. ISCO flash chromatography (80 g silica, 10-60\% 
EtOAc/Hex, 28 min gradient) provided the title compound as a yellow-orange solid (1.936 g, $43 \%$ yield).

${ }^{1} \mathrm{H}$ NMR $\left(500 \mathrm{MHz}, \mathrm{CDCl}_{3}\right) \delta 8.38(\mathrm{~s}, 1 \mathrm{H}), 4.46(\mathrm{q}, J=7.1 \mathrm{~Hz}, 2 \mathrm{H}), 1.42(\mathrm{t}, J=7.1 \mathrm{~Hz}, 3 \mathrm{H}) .{ }^{19} \mathrm{~F}$ NMR (471 MHz, $\left.\mathrm{CDCl}_{3}\right) \delta$-61.02. LRMS (ESI) calculated for $\mathrm{C}_{7} \mathrm{H}_{7} \mathrm{~F}_{3} \mathrm{NO}_{2} \mathrm{~S}[\mathrm{M}+\mathrm{H}]^{+}$226.01, found 225.88 .

\section{Ethyl 5-bromo-2-(trifluoromethyl)thiazole-4-carboxylate (S-37) ${ }^{8}$}<smiles>CCOC(=O)c1nc(C(F)(F)F)sc1Br</smiles>

A $250 \mathrm{~mL}$ round-bottom flask was dried with a heat gun under vacuum. Under $\mathrm{N}_{2}$ atmosphere, anhydrous THF (40 mL) and LDA solution (2 M in THF, $4.70 \mathrm{~mL}, 9.40 \mathrm{mmol}, 1.25$ equiv) were cooled to $-78^{\circ} \mathrm{C}$. A solution of ethyl 2-(trifluoromethyl)thiazole-4-carboxylate $\mathbf{S - 3 6}$ (1.688 g, 7.5 mmol, 1.0 equiv) in $20 \mathrm{~mL}$ THF was added dropwise over 12 minutes, and then stirred at $-78{ }^{\circ} \mathrm{C}$ for thirty minutes. A solution of 1,2-dibromotetrachloroethane (3.663 g, $11.25 \mathrm{mmol}, 1.5$ equiv) in $15 \mathrm{~mL}$ THF was added dropwise over 7 minutes. The reaction was stirred at $-78^{\circ} \mathrm{C}$ for 1 hour, and then at room temperature for 1 hour. The reaction was quenched with saturated aqueous $\mathrm{NH} 4 \mathrm{Cl}(25 \mathrm{~mL})$ and diluted with water $(100 \mathrm{~mL})$, then extracted with EtOAc $(3 \times 75 \mathrm{~mL})$. The combined organic layers were washed with brine, dried over $\mathrm{MgSO}_{4}$, filtered and concentrated. ISCO flash chromatography (40 g silica, 0-40\% EtOAc/Hex, 18 min gradient) provided the title compound as a yellow-orange semi-solid (636 mg, 28\% yield).

${ }^{1} \mathrm{H} \mathrm{NMR}\left(500 \mathrm{MHz}, \mathrm{CDCl}_{3}\right) \delta 4.47$ (q, $\left.J=7.1 \mathrm{~Hz}, 2 \mathrm{H}\right), 1.43(\mathrm{t}, J=7.2 \mathrm{~Hz}, 3 \mathrm{H})$. 19F NMR (471 $\mathrm{MHz}, \mathrm{CDCl} 3) \delta$-61.16. LRMS (ESI) calculated for $\mathrm{C}_{7} \mathrm{H}_{6} \mathrm{BrF}_{3} \mathrm{NO}_{2} \mathrm{~S}[\mathrm{M}+\mathrm{H}]^{+}$303.92, found 303.67, 305.67 .

Ethyl 5-((2-chloro-5-nitropyrimidin-4-yl)(methyl)amino)-2-(trifluoromethyl)thiazole-4carboxylate (S-38)<smiles>CCOC(=O)c1nc(C(F)(F)F)sc1N(C)c1nc(Cl)ncc1[N+](=O)[O-]</smiles> 
To a solution of ethyl 5-bromo-2-(trifluoromethyl)thiazole-4-carboxylate $\mathbf{S - 3 7}$ (632 mg, $2.1 \mathrm{mmol}$, 1.0 equiv) in anhydrous acetonitrile $(10.5 \mathrm{~mL})$ were added methylamine $(2.0 \mathrm{M}$ in THF, $1.30 \mathrm{~mL}$, $2.6 \mathrm{mmol}, 1.24$ equiv) and $\mathrm{DBU}(0.40 \mathrm{~mL}, 2.8 \mathrm{mmol}, 1.3 \mathrm{equiv})$. The mixture was heated to 80 ${ }^{\circ} \mathrm{C}$ for 2 hours, and then cooled to room temperature. The reaction was diluted with $50 \mathrm{~mL}$ each EtOAc and water. The organic layer was removed and the aqueous was extracted with EtOAc (3x50 mL). The combined organic layers were washed with brine, dried over $\mathrm{MgSO}_{4}$, filtered and concentrated. UPLC-MS analysis showed ethyl 5-(methylamino)-2-(trifluoromethyl)thiazole-4carboxylate in approx. 30\% yield, and the crude mixture was carried on to the next step without purification. LRMS (ESI) calculated for $\mathrm{C}_{8} \mathrm{H}_{10} \mathrm{~F}_{3} \mathrm{~N}_{2} \mathrm{O}_{2} \mathrm{~S}[\mathrm{M}+\mathrm{H}]^{+} 255.04$, found 254.87 .

The entirety of the crude methylamination mixture was subjected to General Procedure B on 1.5 mmol scale, using 2,4-dichloro-5-nitropyrimidine (449.3 $\mathrm{mg}, 2.30 \mathrm{mmol}, 1.6$ equiv) as the electrophile. The reaction was stirred at room temperature overnight. ISCO flash chromatography (24 g silica, 0 to $40 \% \mathrm{EtOAc/hexanes} \mathrm{gradient,} 20$ minutes) provided the title compound as a clear yellow oil (143.7 $\mathrm{mg}, 16 \%$ yield over 2 steps).

${ }^{1} \mathrm{H}$ NMR $\left(500 \mathrm{MHz}, \mathrm{CDCl}_{3}\right) \delta 8.71(\mathrm{~s}, 1 \mathrm{H}), 4.29(\mathrm{q}, J=7.1 \mathrm{~Hz}, 2 \mathrm{H}), 3.71(\mathrm{~s}, 3 \mathrm{H}), 1.28(\mathrm{t}, J=7.2$ $\mathrm{Hz}, 3 \mathrm{H}) .{ }^{19} \mathrm{~F} \mathrm{NMR}\left(471 \mathrm{MHz}, \mathrm{CDCl}_{3}\right) \delta$-61.82. LRMS (ESI) calculated for $\mathrm{C}_{12} \mathrm{H}_{10} \mathrm{ClF}_{3} \mathrm{~N}_{5} \mathrm{O}_{4} \mathrm{~S}$ $[\mathrm{M}+\mathrm{H}]^{+}$412.00, found 411.87 .

\section{6-chloro-4-methyl-2-(trifluoromethyl)-4,9-dihydro-10H-pyrimido[5,4-b]thiazolo[5,4- e][1,4]diazepin-10-one (S-39)}<smiles>CN1c2nc(Cl)ncc2NC(=O)c2nc(C(F)(F)F)sc21</smiles>

General Procedure C was followed on $0.33 \mathrm{mmol}$ scale using S-38 (136.2 mg). Modified isolation: after removing iron, the reaction was diluted with water $(5 \mathrm{~mL})$ and extracted with DCM $(5 \times 5$ $\mathrm{mL})$. The combined organic layers were washed with sat. aqueous $\mathrm{NaHCO}_{3}(3 \times 15 \mathrm{~mL})$ and then brine, dried over $\mathrm{MgSO}_{4}$, filtered and concentrated to provide the title compound as a yellow solid (97.1 mg, 88\% yield). 
${ }^{1} \mathrm{H}$ NMR (500 MHz, DMSO- $\left.d_{6}\right) \delta 10.30(\mathrm{~s}, 1 \mathrm{H}), 8.20(\mathrm{~s}, 1 \mathrm{H}), 3.47(\mathrm{~s}, 3 \mathrm{H}) .{ }^{19} \mathrm{~F}$ NMR $(471 \mathrm{MHz}$, DMSO) $\delta$-60.55. LRMS (ESI) calculated for $\mathrm{C}_{10} \mathrm{H}_{6} \mathrm{ClF}_{3} \mathrm{~N}_{5} \mathrm{OS}[\mathrm{M}+\mathrm{H}]^{+} 335.99$, found 335.77 .

\section{6-chloro-4,9-dimethyl-2-(trifluoromethyl)-4,9-dihydro-10H-pyrimido[5,4-b]thiazolo[5,4- e][1,4]diazepin-10-one (S-40)}<smiles>CN1C(=O)c2nc(C(F)(F)F)sc2N(C)c2nc(Cl)ncc21</smiles>

General Procedure D was followed on $0.24 \mathrm{mmol}$ scale using S-39 $(81.3 \mathrm{mg})$ and iodomethane (30.0 $\mu \mathrm{L}, 2.0$ equiv) as the electrophile, with a reaction time of 2 hours. The reaction was then quenched with water $(5 \mathrm{~mL})$ and extracted with DCM $(5 \times 5 \mathrm{~mL})$. The combined organic layers were washed three times with water, once with brine, dried over $\mathrm{MgSO}_{4}$, filtered and concentrated. ISCO flash chromatography (12 g silica, $0-5 \% \mathrm{MeOH} / \mathrm{DCM}, 12 \mathrm{~min}$ gradient) provided the title compound as a yellow solid (32.6 mg, 39\% yield).

${ }^{1} \mathrm{H} \mathrm{NMR}\left(500 \mathrm{MHz}, \mathrm{CDCl}_{3}\right) \delta 8.33(\mathrm{~s}, 1 \mathrm{H}), 3.54(\mathrm{~s}, 3 \mathrm{H}), 3.47(\mathrm{~s}, 3 \mathrm{H}) .{ }^{19} \mathrm{~F} \mathrm{NMR}\left(471 \mathrm{MHz}, \mathrm{CDCl}_{3}\right)$ $\delta$-61.28. LRMS (ESI) calculated for $\mathrm{C}_{11} \mathrm{H}_{8} \mathrm{ClF}_{3} \mathrm{~N}_{5} \mathrm{OS}[\mathrm{M}+\mathrm{H}]^{+} 350.01$, found 349.77 .

Synthesis of 2-methyloxazole core<smiles>CCOC(=O)c1coc(C)n1</smiles>

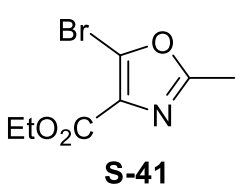

$29 \%$ yield
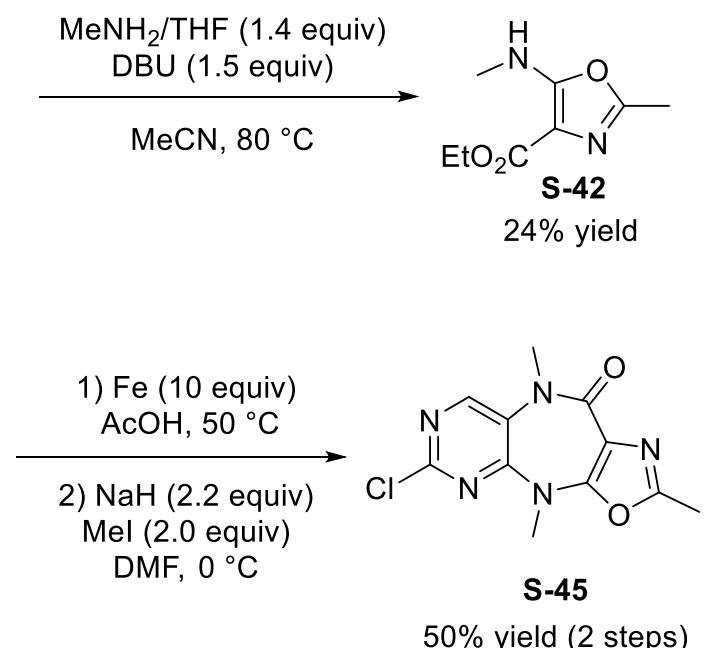

$50 \%$ yield (2 steps)
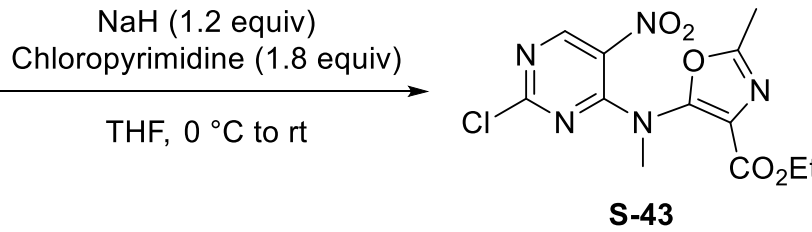

$19 \%$ yield 
Ethyl 5-bromo-2-methyloxazole-4-carboxylate (S-41)<smiles>CCOC(=O)c1nc(C)oc1Br</smiles>

General Procedure A was followed on 15.0 mmol scale, using ethyl 2-methyloxazole-4carboxylate. ISCO flash chromatography (40 g silica, 20-70\% EtOAc/hex, 11 min gradient then 3 min isocratic hold) provided the title compound as a yellow solid (990 mg, 29\% yield).

${ }^{1} \mathrm{H}$ NMR $\left(500 \mathrm{MHz}, \mathrm{CDCl}_{3}\right) \delta 4.40$ (q, $\left.J=7.2 \mathrm{~Hz}, 2 \mathrm{H}\right), 2.77(\mathrm{~s}, 3 \mathrm{H}), 1.39$ (t, $\left.J=7.1 \mathrm{~Hz}, 3 \mathrm{H}\right)$. LRMS (ESI) calculated for $\mathrm{C}_{7} \mathrm{H}_{9} \mathrm{BrNO}_{3}[\mathrm{M}+\mathrm{H}]^{+}$233.98, found 233.88, 235.88.

\section{Ethyl 2-methyl-5-(methylamino)oxazole-4-carboxylate (S-42)}<smiles>CCOC(=O)c1nc(C)oc1NC</smiles>

To a solution of ethyl 5-bromo-2-methyloxazole-4-carboxylate $\mathbf{S - 4 1}$ (970 mg, 4.15 mmol, 1.0 equiv) in anhydrous acetonitrile $(14 \mathrm{~mL})$ were added methylamine $(2.0 \mathrm{M}$ in THF, $3.1 \mathrm{~mL}, 6.2$ mmol, 1.5 equiv) and DBU ( $0.87 \mathrm{~mL}, 6.2 \mathrm{mmol}, 1.5$ equiv). The mixture was heated to $80{ }^{\circ} \mathrm{C}$ for 3 hours, and then cooled to room temperature. The reaction was diluted with $50 \mathrm{~mL}$ each EtOAc and water. The organic layer was removed and the aqueous was extracted with EtOAc $(3 \times 50$ $\mathrm{mL}$ ). The combined organic layers were washed with brine, dried over $\mathrm{MgSO}_{4}$, filtered and concentrated. ISCO flash chromatography (24 g silica, 25-80\% EtOAc/hex, $16 \mathrm{~min}$ ) provided the title compound (232 $\mathrm{mg}, 24 \%$ yield) as a yellow solid.

LRMS (ESI) calculated for $\mathrm{C}_{8} \mathrm{H}_{13} \mathrm{~N}_{2} \mathrm{O}_{3}[\mathrm{M}+\mathrm{H}]^{+}$185.09, found 185.09.

Ethyl 5-((2-chloro-5-nitropyrimidin-4-yl)(methyl)amino)-2-methyloxazole-4-carboxylate (S43)<smiles>CCOC(=O)c1nc(C)oc1N(C)c1nc(Cl)ncc1[N+](=O)[O-]</smiles>

General Procedure B was followed on $1.25 \mathrm{mmol}$ scale using S-42 (232 mg) and 2,4-dichloro-5nitropyrimidine (435 mg, $2.25 \mathrm{mmol}, 1.8$ equiv) as the electrophile. ISCO flash chromatography 
(12 $\mathrm{g}$ silica, 20-75\% EtOAc/hex, $15 \mathrm{~min}$ ) provided the title compound (80.4 $\mathrm{mg}, 19 \%$ yield) as a viscous yellow oil.

${ }^{1} \mathrm{H} \mathrm{NMR}\left(500 \mathrm{MHz}, \mathrm{CDCl}_{3}\right) \delta 8.75(\mathrm{~s}, 1 \mathrm{H}), 4.24(\mathrm{q}, J=7.2 \mathrm{~Hz}, 2 \mathrm{H}), 3.64(\mathrm{~s}, 3 \mathrm{H}), 2.50(\mathrm{~s}, 3 \mathrm{H})$, $1.25(\mathrm{t}, J=7.1 \mathrm{~Hz}, 3 \mathrm{H})$. LRMS (ESI) calculated for $\mathrm{C}_{12} \mathrm{H}_{13} \mathrm{ClN}_{5} \mathrm{O}_{5}[\mathrm{M}+\mathrm{H}]^{+} 342.06$, found 341.77 .

6-chloro-2,4-dimethyl-4,9-dihydro-10H-oxazolo[5,4-e]pyrimido[5,4-b][1,4]diazepin-10-one (S-44)<smiles>Cc1nc2c(o1)N(C)c1nc(Cl)ncc1NC2=O</smiles>

General Procedure C was followed on $0.24 \mathrm{mmol}$ scale using ethyl 5-((2-chloro-5nitropyrimidin-4-yl)(methyl)amino)-2-methyloxazole-4-carboxylate S-43 (80.4 mg). Modified isolation: after removing iron, the reaction was diluted with water $(5 \mathrm{~mL})$ and extracted with DCM $(5 \times 5 \mathrm{~mL})$. The combined organic layers were washed with sat. aqueous $\mathrm{NaHCO}_{3}(3 \times 15$ $\mathrm{mL}$ ) and then brine, dried over $\mathrm{MgSO}_{4}$, filtered and concentrated to provide the title compound as a yellow solid (48.8 $\mathrm{mg}, 77 \%$ yield).

${ }^{1} \mathrm{H}$ NMR (500 MHz, DMSO-d6) $\delta 9.51(\mathrm{~s}, 1 \mathrm{H}), 8.01(\mathrm{~s}, 1 \mathrm{H}), 2.34(\mathrm{~s}, 3 \mathrm{H})\left(\mathrm{N}-\mathrm{CH}_{3}\right.$ covered by water peak). LRMS (ESI) calculated for $\mathrm{C}_{10} \mathrm{H}_{9} \mathrm{ClN}_{5} \mathrm{O}_{2}[\mathrm{M}+\mathrm{H}]^{+}$266.04, found 265.87 .

6-chloro-2,4,9-trimethyl-4,9-dihydro-10H-oxazolo[5,4-e]pyrimido[5,4-b][1,4]diazepin-10one $(\mathrm{S}-45)$<smiles>Cc1nc2c(o1)N(C)c1nc(Cl)ncc1N(C)C2=O</smiles>

General Procedure D was followed on $0.135 \mathrm{mmol}$ scale using 6-chloro-2,4-dimethyl-4,9dihydro-10H-oxazolo[5,4-e]pyrimido[5,4-b][1,4]diazepin-10-one $\mathbf{S - 4 4 ~ ( 3 6 . 0 ~ m g ) ~ a n d ~}$ iodomethane $(17.00 \mu \mathrm{L}, 0.270 \mathrm{mmol}, 2.0$ equiv) as the electrophile. The reaction was then quenched with water $(5 \mathrm{~mL})$ and extracted with DCM $(5 \times 5 \mathrm{~mL})$. The combined organic layers 
were washed three times with water, once with brine, dried over $\mathrm{MgSO}_{4}$, filtered and concentrated. ISCO flash chromatography (4 g silica, $0-10 \% \mathrm{MeOH} / \mathrm{DCM}, 12$ min gradient) to provide the title compound as a yellow solid $(24.8 \mathrm{mg}, 66 \%$ yield).

LRMS (ESI) calculated for $\mathrm{C}_{11} \mathrm{H}_{11} \mathrm{ClN}_{5} \mathrm{O}_{2}[\mathrm{M}+\mathrm{H}]^{+} 280.06$, found 279.77 .

\section{$\underline{\text { Final Compounds }}$}

2,4-dimethyl-6-((4-(4-methylpiperazin-1-yl)phenyl)amino)-4,9-dihydro-10H-pyrimido[5,4b]thiazolo[5,4-e][1,4]diazepin-10-one (S-46, BJG-01-177)

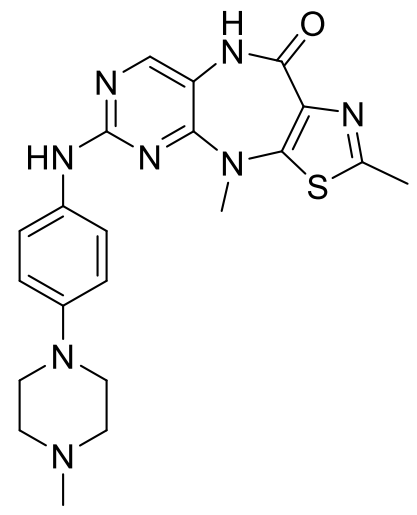

General Procedure E was run on $0.05 \mathrm{mmol}$ scale using 4-(4-methylpiperazin-1-yl)aniline (14.0 $\mathrm{mg}, 0.075 \mathrm{mmol}, 1.5$ equiv). The reaction mixture was purified by reverse-phase prep HPLC (100$40 \% \mathrm{H}_{2} \mathrm{O} / \mathrm{MeOH}, 20 \mathrm{~mL} / \mathrm{min}, 45 \mathrm{~min}$ ). Lyophilization from $\mathrm{H}_{2} \mathrm{O} / \mathrm{MeCN}$ provided the title compound as a yellow powder ( $8.1 \mathrm{mg}$ TFA salt).

${ }^{1} \mathrm{H}-\mathrm{NMR}\left(500 \mathrm{MHz}, \mathrm{DMSO}-d_{6}\right) \delta 9.96(\mathrm{~s}, 1 \mathrm{H}), 9.53(\mathrm{~s}, 1 \mathrm{H}), 9.38(\mathrm{~s}, 1 \mathrm{H}), 7.98$ (s, 1H), 7.57 (d, $J$ $=8.8 \mathrm{~Hz}, 2 \mathrm{H}), 6.95(\mathrm{~d}, J=8.8 \mathrm{~Hz}, 2 \mathrm{H}), 3.72(\mathrm{~d}, J=13.2 \mathrm{~Hz}, 2 \mathrm{H}), 3.36(\mathrm{~s}, 3 \mathrm{H}), 3.16(\mathrm{~m}, 2 \mathrm{H}), 2.88$ (m, 5H), 2.53 (s, 3H). LRMS (ESI) calculated for $\mathrm{C}_{21} \mathrm{H}_{25} \mathrm{~N}_{8} \mathrm{OS}[\mathrm{M}+\mathrm{H}]^{+} 437.19$, found 437.28. 
<smiles>Cc1nc2c(s1)N(C)c1nc(Nc3ccc(N4CCC(O)CC4)cc3)ncc1NC2=O</smiles>

General Procedure E was run on $0.05 \mathrm{mmol}$ scale using 1-(4-aminophenyl)piperidin-4-ol (14.4 mg, $0.075 \mathrm{mmol}, 1.5$ equiv). The reaction mixture was purified by reverse-phase prep HPLC (100$40 \% \mathrm{H}_{2} \mathrm{O} / \mathrm{MeOH}, 20 \mathrm{~mL} / \mathrm{min}, 45 \mathrm{~min}$ ). Lyophilization from $\mathrm{H}_{2} \mathrm{O} / \mathrm{MeCN}$ provided the title compound as a yellow powder (6.3 mg TFA salt).

LRMS (ESI) calculated for $\mathrm{C}_{21} \mathrm{H}_{24} \mathrm{~N}_{7} \mathrm{O}_{2} \mathrm{~S}[\mathrm{M}+\mathrm{H}]^{+}$438.17, found 438.28.

6-((2-methoxy-4-(4-(4-methylpiperazin-1-yl)piperidin-1-yl)phenyl)amino)-2,4-dimethyl-4,9dihydro-10H-pyrimido[5,4-b]thiazolo[5,4-e][1,4]diazepin-10-one (1, BJG-01-181)<smiles>COc1cc(N2CCC(N3CCN(C)CC3)CC2)ccc1Nc1ncc2c(n1)N(C)c1sc(C)nc1C(=O)N2</smiles>

General Procedure E was run on $0.05 \mathrm{mmol}$ scale using 2-methoxy-4-(4-(4-methylpiperazin-1yl)piperidin-1-yl)aniline $(21.1 \mathrm{mg}, 0.070 \mathrm{mmol}, 1.4$ equiv). The reaction mixture was purified by reverse-phase prep HPLC (100-40\% $\left.\mathrm{H}_{2} \mathrm{O} / \mathrm{MeOH}, 20 \mathrm{~mL} / \mathrm{min}, 45 \mathrm{~min}\right)$. Lyophilization from $\mathrm{H}_{2} \mathrm{O} / \mathrm{MeCN}$ provided the title compound as a beige powder ( $8.8 \mathrm{mg}$ TFA salt). 
${ }^{1} \mathrm{H}$ NMR $\left(500 \mathrm{MHz}, \mathrm{DMSO}-d_{6}\right) \delta 9.51(\mathrm{~s}, 1 \mathrm{H}), 7.95(\mathrm{~s}, 1 \mathrm{H}), 7.93(\mathrm{~s}, 1 \mathrm{H}), 7.72(\mathrm{~d}, J=8.7 \mathrm{~Hz}, 1 \mathrm{H})$, $6.70(\mathrm{~s}, 1 \mathrm{H}), 6.56(\mathrm{~d}, J=8.6 \mathrm{~Hz}, 1 \mathrm{H}), 3.81(\mathrm{~s}, 3 \mathrm{H}), 3.79-3.65(\mathrm{~m}, 8 \mathrm{H}), 3.31(\mathrm{~s}, 3 \mathrm{H}), 2.76(\mathrm{~s}, 3 \mathrm{H})$, $2.53(\mathrm{~s}, 3 \mathrm{H}), 2.04-1.92(\mathrm{~m}, 2 \mathrm{H}), 1.71-1.59(\mathrm{~m}, 2 \mathrm{H})$. LRMS (ESI) calculated for $\mathrm{C}_{27} \mathrm{H}_{36} \mathrm{~N}_{9} \mathrm{O}_{2} \mathrm{~S}$ $[\mathrm{M}+\mathrm{H}]^{+} 550.27$, found 550.39 .

6-((3-methoxy-4-(4-methylpiperazin-1-yl)phenyl)amino)-2,4-dimethyl-4,9-dihydro-10Hpyrimido[5,4-b]thiazolo[5,4-e][1,4]diazepin-10-one (S-48, BJG-02-015)<smiles>COc1cc(Nc2ncc3c(n2)N(C)c2sc(C)nc2C(=O)N3)ccc1N1CCN(C)CC1</smiles>

General Procedure E was run on 0.05 mmol scale using 3-methoxy-4-(4-methylpiperazin-1yl)aniline ( $16.5 \mathrm{mg}, 0.075 \mathrm{mmol}, 1.5$ equiv). The reaction mixture was purified by reverse-phase prep HPLC (90-40\% $\left.\mathrm{H}_{2} \mathrm{O} / \mathrm{MeOH}, 20 \mathrm{~mL} / \mathrm{min}, 45 \mathrm{~min}\right)$. Lyophilization from $\mathrm{H}_{2} \mathrm{O} / \mathrm{MeCN}$ provided the title compound as a yellow powder (16.0 mg TFA salt).

${ }^{1} \mathrm{H}$ NMR $\left(500 \mathrm{MHz}, \mathrm{DMSO}-d_{6}\right) \delta 9.62(\mathrm{~s}, 1 \mathrm{H}), 9.55(\mathrm{~s}, 1 \mathrm{H}), 9.45(\mathrm{~s}, 1 \mathrm{H}), 8.00(\mathrm{~s}, 1 \mathrm{H}), 7.46(\mathrm{~d}, J$ $=2.3 \mathrm{~Hz}, 1 \mathrm{H}), 7.21(\mathrm{dd}, J=8.6,2.4 \mathrm{~Hz}, 1 \mathrm{H}), 6.87(\mathrm{~d}, J=8.6 \mathrm{~Hz}, 1 \mathrm{H}), 3.79(\mathrm{~s}, 3 \mathrm{H}), 3.48(\mathrm{~d}, J=$ $11.8 \mathrm{~Hz}, 2 \mathrm{H}), 3.40$ (s, 5H), 3.19 (q, $J=11.0 \mathrm{~Hz}, 2 \mathrm{H}), 2.91-2.80(\mathrm{~m}, 5 \mathrm{H}), 2.54$ (s, 3H). LRMS (ESI) calculated for $\mathrm{C}_{22} \mathrm{H}_{27} \mathrm{~N}_{8} \mathrm{O}_{2} \mathrm{~S}[\mathrm{M}+\mathrm{H}]^{+} 467.20$, found 467.38 . 


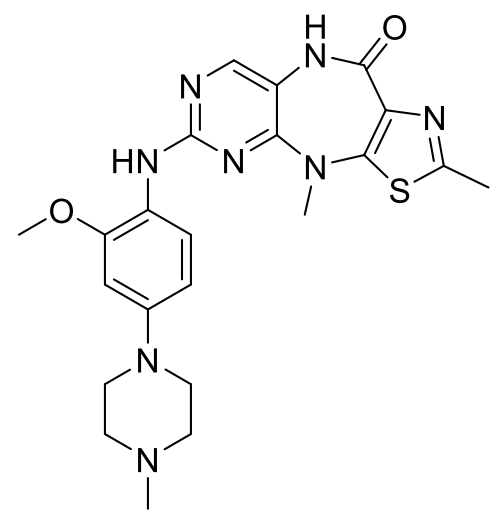

General Procedure E was run on $0.08 \mathrm{mmol}$ scale using 2-methoxy-4-(4-methylpiperazin-1yl)aniline (27.2 mg, $0.120 \mathrm{mmol}, 1.5$ equiv). The reaction mixture was purified by reverse-phase prep HPLC (100-30\% $\left.\mathrm{H}_{2} \mathrm{O} / \mathrm{MeCN}, 20 \mathrm{~mL} / \mathrm{min}, 28 \mathrm{~min}\right)$. Lyophilization from $\mathrm{H}_{2} \mathrm{O} / \mathrm{MeCN}$ provided the title compound as a light brown powder (3.9 mg TFA salt).

${ }^{1} \mathrm{H}$ NMR (500 MHz, DMSO-d6) $\delta 9.87$ (s, 1H), 9.51 (s, 1H), 7.96 (s, 1H), 7.93 (s, 1H), 7.74 (d, $J$ $=8.8 \mathrm{~Hz}, 1 \mathrm{H}), 6.70(\mathrm{~d}, J=2.6 \mathrm{~Hz}, 1 \mathrm{H}), 6.54(\mathrm{dd}, J=8.8,2.6 \mathrm{~Hz}, 1 \mathrm{H}), 3.82(\mathrm{~s}, 3 \mathrm{H}), 3.53(\mathrm{~d}, J=$ $12.4 \mathrm{~Hz}, 2 \mathrm{H}), 3.31(\mathrm{~s}, 3 \mathrm{H}), 3.24-3.11(\mathrm{~m}, 2 \mathrm{H}), 2.93(\mathrm{t}, J=12.5 \mathrm{~Hz}, 2 \mathrm{H}), 2.87(\mathrm{~s}, 3 \mathrm{H}), 2.53(\mathrm{~s}$, $3 \mathrm{H})$. LRMS (ESI) calculated for $\mathrm{C}_{22} \mathrm{H}_{27} \mathrm{~N}_{8} \mathrm{O}_{2} \mathrm{~S}[\mathrm{M}+\mathrm{H}]^{+} 467.20$, found 467.18.

6-((2-methoxy-4-(4-(4-methylpiperazin-1-yl)piperidin-1-yl)phenyl)amino)-2,4,9-trimethyl4,9-dihydro-10H-pyrimido[5,4-b]thiazolo[5,4-e][1,4]diazepin-10-one (4, BJG-03-025)<smiles>COc1cc(N2CCC(N3CCN(C)CC3)CC2)ccc1Nc1ncc2c(n1)N(C)c1sc(C)nc1C(=O)N2C</smiles>

General Procedure E was run on $0.05 \mathrm{mmol}$ scale using 2-methoxy-4-(4-(4-methylpiperazin-1yl)piperidin-1-yl)aniline (21.6 mg, $0.07 \mathrm{mmol}, 1.4$ equiv). The reaction mixture was purified by 
reverse-phase prep HPLC (100-50\% $\left.\mathrm{H}_{2} \mathrm{O} / \mathrm{MeCN}, 20 \mathrm{~mL} / \mathrm{min}, 45 \mathrm{~min}\right)$. Lyophilization from $\mathrm{H}_{2} \mathrm{O} / \mathrm{MeCN}$ provided the title compound as a white powder (4.2 mg TFA salt).

${ }^{1} \mathrm{H}$ NMR $\left(500 \mathrm{MHz}\right.$, DMSO- $\left.d_{6}\right) \delta 8.30(\mathrm{~s}, 1 \mathrm{H}), 8.06(\mathrm{~s}, 1 \mathrm{H}), 7.64(\mathrm{~d}, J=8.7 \mathrm{~Hz}, 1 \mathrm{H}), 6.61(\mathrm{~d}, J=$ $2.6 \mathrm{~Hz}, 1 \mathrm{H}), 6.48(\mathrm{dd}, J=8.8,2.6 \mathrm{~Hz}, 1 \mathrm{H}), 3.79(\mathrm{~s}, 3 \mathrm{H}), 3.68(\mathrm{~d}, J=12.2 \mathrm{~Hz}, 2 \mathrm{H}), 3.28(\mathrm{~s}, 3 \mathrm{H})$, $3.26(\mathrm{~s}, 3 \mathrm{H}), 2.63(\mathrm{td}, J=12.1,2.3 \mathrm{~Hz}, 3 \mathrm{H}), 2.53(\mathrm{~s}, 4 \mathrm{H}), 2.38(\mathrm{~m}, 4 \mathrm{H}), 2.22(\mathrm{~s}, 3 \mathrm{H}), 1.84(\mathrm{~d}, J=$ $12.3 \mathrm{~Hz}, 2 \mathrm{H}), 1.58-1.44(\mathrm{~m}, 2 \mathrm{H})$. LRMS (ESI) calculated for $\mathrm{C}_{28} \mathrm{H}_{38} \mathrm{~N}_{9} \mathrm{O}_{2} \mathrm{~S}[\mathrm{M}+\mathrm{H}]^{+} 564.29$, found 564.49 .

6-((2-methoxy-4-(4-methylpiperazin-1-yl)phenyl)amino)-2,4,9-trimethyl-4,9-dihydro-10Hpyrimido[5,4-b]thiazolo[5,4-e][1,4]diazepin-10-one (11, BJG-03-161)<smiles>COc1cc(N2CCN(C)CC2)ccc1Nc1ncc2c(n1)N(C)c1sc(C)nc1C(=O)N2C</smiles>

General Procedure E was run on $0.055 \mathrm{mmol}$ scale using 2-methoxy-4-(4-methylpiperazin-1yl)aniline ( $21.7 \mathrm{mg}, 0.098 \mathrm{mmol}, 1.8$ equiv). The reaction mixture was purified by reverse-phase prep HPLC (100-40\% $\left.\mathrm{H}_{2} \mathrm{O} / \mathrm{MeCN}, 20 \mathrm{~mL} / \mathrm{min}, 45 \mathrm{~min}\right)$. The material was further purified by prep TLC (10\% MeOH/DCM). Lyophilization from $\mathrm{H}_{2} \mathrm{O} / \mathrm{MeCN}$ provided the title compound as a light yellow powder (10.1 mg TFA salt).

${ }^{1} \mathrm{H}$ NMR $\left(500 \mathrm{MHz}\right.$, DMSO- $\left.d_{6}\right) \delta 9.77(\mathrm{~s}, 1 \mathrm{H}), 8.31(\mathrm{~s}, 1 \mathrm{H}), 8.12(\mathrm{~s}, 1 \mathrm{H}), 7.72(\mathrm{~d}, J=8.7 \mathrm{~Hz}, 1 \mathrm{H})$, $6.69(\mathrm{~d}, J=2.6 \mathrm{~Hz}, 1 \mathrm{H}), 6.54(\mathrm{dd}, J=8.7,2.6 \mathrm{~Hz}, 1 \mathrm{H}), 3.81$ (s, 3H), $3.32(\mathrm{br} \mathrm{s}, 8 \mathrm{H}), 3.29$ (s, $3 \mathrm{H})$, $3.27(\mathrm{~s}, 3 \mathrm{H}), 2.79$ (s, 3H), 2.53 (s, 3H). LRMS (ESI) calculated for $\mathrm{C}_{23} \mathrm{H}_{29} \mathrm{~N}_{8} \mathrm{O}_{2} \mathrm{~S}[\mathrm{M}+\mathrm{H}]^{+} 481.21$, found 480.88 . 


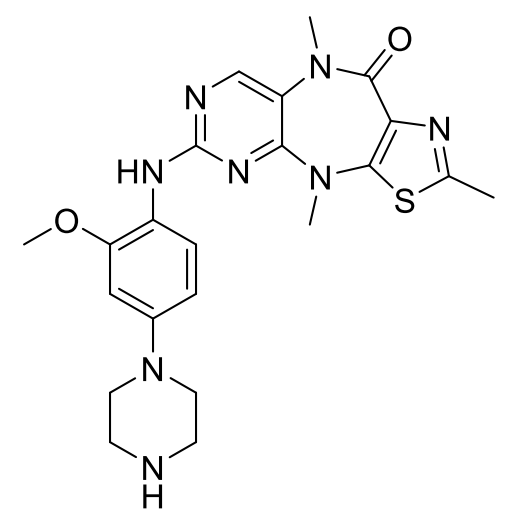

General Procedure E was run on $0.13 \mathrm{mmol}$ scale using tert-butyl 4-(4-amino-3methoxyphenyl)piperazine-1-carboxylate. ISCO flash chromatography (12 g silica, 0-10\% $\mathrm{MeOH} / \mathrm{DCM}, 12 \mathrm{~min}$ gradient) provided tert-butyl 4-(3-methoxy-4-((2,4,9-trimethyl-10-oxo9,10-dihydro-4H-pyrimido[5,4-b] thiazolo[5,4-e][1,4]diazepin-6-yl)amino)phenyl)piperazine-1carboxylate as a yellow solid (29.4 mg, $40 \%$ yield).

${ }^{1} \mathrm{H}$ NMR (500 MHz, DMSO- $\left.d_{6}\right) \delta 8.31(\mathrm{~s}, 1 \mathrm{H}), 8.09(\mathrm{~s}, 1 \mathrm{H}), 7.68(\mathrm{~d}, J=8.7 \mathrm{~Hz}, 1 \mathrm{H}), 6.66(\mathrm{~d}, J=$ $2.6 \mathrm{~Hz}, 1 \mathrm{H}), 6.51(\mathrm{dd}, J=8.8,2.6 \mathrm{~Hz}, 1 \mathrm{H}), 3.80$ (s, 3H), 3.46 (t, $J=5.1 \mathrm{~Hz}, 4 \mathrm{H}), 3.29$ (s, 3H), 3.26 $(\mathrm{s}, 3 \mathrm{H}), 3.07(\mathrm{t}, J=5.2 \mathrm{~Hz}, 4 \mathrm{H}), 2.53(\mathrm{~s}, 3 \mathrm{H}), 1.42(\mathrm{~s}, 9 \mathrm{H})$. LRMS (ESI) calculated for $\mathrm{C}_{27} \mathrm{H}_{35} \mathrm{~N}_{8} \mathrm{O}_{4} \mathrm{~S}$ $[\mathrm{M}+\mathrm{H}]^{+} 567.25$, found 567.39 .

The Boc-protected intermediate ( $29.4 \mathrm{mg}, 0.052 \mathrm{mmol}, 1.0$ equiv) was dissolved in DCM (0.8 mL) and TFA $(0.2 \mathrm{~mL})$. The mixture was stirred at room temp for 3 hours; UPLC-MS analysis showed complete deprotection. Volatiles were removed in vacuo; lyophilization from $\mathrm{H}_{2} \mathrm{O} / \mathrm{MeCN}$ provided the title compound as a yellow powder (44.4 mg, 2xTFA salt).

${ }^{1} \mathrm{H}$ NMR $\left(500 \mathrm{MHz}\right.$, DMSO- $\left.d_{6}\right) \delta 8.78(\mathrm{~s}, 2 \mathrm{H}), 8.31(\mathrm{~s}, 1 \mathrm{H}), 8.14(\mathrm{~s}, 1 \mathrm{H}), 7.72(\mathrm{~d}, J=8.7 \mathrm{~Hz}, 1 \mathrm{H})$, $6.69(\mathrm{~d}, J=2.6 \mathrm{~Hz}, 1 \mathrm{H}), 6.54(\mathrm{dd}, J=8.8,2.6 \mathrm{~Hz}, 1 \mathrm{H}), 3.81(\mathrm{~s}, 3 \mathrm{H}), 3.35-3.31(\mathrm{~m}, 4 \mathrm{H}), 3.30(\mathrm{~s}$, $3 \mathrm{H}$ ), $3.27(\mathrm{~s}, 3 \mathrm{H}), 3.26-3.20(\mathrm{~m}, 4 \mathrm{H}), 2.53$ (s, 3H). LRMS (ESI) calculated for $\mathrm{C}_{22} \mathrm{H}_{27} \mathrm{~N}_{8} \mathrm{O}_{2} \mathrm{~S}$ $[\mathrm{M}+\mathrm{H}]^{+} 467.20$, found 466.88 . 
<smiles>COc1cc(N2CCN(C)CC2=O)ccc1Nc1ncc2c(n1)N(C)C(=O)c1nc(C)sc1N2C</smiles>

General Procedure E was run on 0.05 mmol scale using 1-(4-amino-3-methoxyphenyl)-4methylpiperazin-2-one (16.0 $\mathrm{mg}, 0.065 \mathrm{mmol}, 1.3$ equiv). The reaction mixture was purified by reverse-phase prep HPLC (100-50\% $\left.\mathrm{H}_{2} \mathrm{O} / \mathrm{MeCN}, 20 \mathrm{~mL} / \mathrm{min}, 45 \mathrm{~min}\right)$. Lyophilization from $\mathrm{H}_{2} \mathrm{O} / \mathrm{MeCN}$ provided the title compound as a white powder (4.8 mg TFA salt).

${ }^{1} \mathrm{H}$ NMR $\left(500 \mathrm{MHz}, \mathrm{DMSO}-d_{6}\right) \delta 8.39(\mathrm{~s}, 1 \mathrm{H}), 8.22(\mathrm{~s}, 1 \mathrm{H}), 8.02(\mathrm{~d}, J=8.6 \mathrm{~Hz}, 1 \mathrm{H}), 7.01(\mathrm{~d}, J=$ $2.2 \mathrm{~Hz}, 1 \mathrm{H}), 6.88(\mathrm{dd}, J=8.6,2.2 \mathrm{~Hz}, 1 \mathrm{H}), 3.83(\mathrm{~s}, 3 \mathrm{H}), 3.65(\mathrm{dd}, J=6.3,4.6 \mathrm{~Hz}, 2 \mathrm{H}), 3.34$ (s, $2 \mathrm{H}), 3.28(\mathrm{~s}, 3 \mathrm{H}), 3.10(\mathrm{~s}, 2 \mathrm{H}), 2.75-2.70(\mathrm{~m}, 2 \mathrm{H}), 2.54(\mathrm{~s}, 3 \mathrm{H}), 2.29(\mathrm{~s}, 3 \mathrm{H})$. LRMS (ESI) calculated for $\mathrm{C}_{23} \mathrm{H}_{27} \mathrm{~N}_{8} \mathrm{O}_{3} \mathrm{~S}[\mathrm{M}+\mathrm{H}]^{+} 495.19$, found 494.88 .

6-((2-methoxy-4-(4-(methylsulfonyl)piperazin-1-yl)phenyl)amino)-2,4,9-trimethyl-4,9dihydro-10H-pyrimido[5,4-b]thiazolo[5,4-e][1,4]diazepin-10-one (14, BJG-03-181)<smiles>COc1cc(N2CCN(S(C)(=O)=O)CC2)ccc1Nc1ncc2c(n1)N(C)c1sc(C)nc1C(=O)N2C</smiles> 
General Procedure E was run on 0.05 mmol scale using 2-methoxy-4-(4(methylsulfonyl)piperazin-1-yl)aniline $(21.8 \mathrm{mg}, 0.065 \mathrm{mmol}, 1.3 \mathrm{equiv})$. The reaction mixture was purified by reverse-phase prep HPLC (100-50\% $\left.\mathrm{H}_{2} \mathrm{O} / \mathrm{MeCN}, 20 \mathrm{~mL} / \mathrm{min}, 45 \mathrm{~min}\right)$. Further purification by prep TLC (10\% $\left.\mathrm{MeOH} / 2 \% \mathrm{NEt}_{3} / \mathrm{DCM}\right)$ and lyophilization from $\mathrm{H}_{2} \mathrm{O} / \mathrm{MeCN}$ provided the title compound as a white powder (5.1 $\mathrm{mg}$ free base).

${ }^{1} \mathrm{H}$ NMR (500 MHz, DMSO-d6) $\delta 8.31(\mathrm{~s}, 1 \mathrm{H}), 8.10$ (s, 1H), $7.71(\mathrm{~d}, \mathrm{~J}=8.8 \mathrm{~Hz}, 1 \mathrm{H}), 6.68(\mathrm{~d}, \mathrm{~J}=$ $2.6 \mathrm{~Hz}, 1 \mathrm{H}), 6.53(\mathrm{dd}, \mathrm{J}=8.8,2.6 \mathrm{~Hz}, 1 \mathrm{H}), 3.81(\mathrm{~s}, 3 \mathrm{H}), 3.30(\mathrm{~s}, 3 \mathrm{H}), 3.27(\mathrm{~s}, 3 \mathrm{H}), 3.25$ - 3.20 (m, $8 \mathrm{H}), 2.93(\mathrm{~s}, 3 \mathrm{H}), 2.53(\mathrm{~s}, 3 \mathrm{H})$. LRMS (ESI) calculated for $\mathrm{C}_{23} \mathrm{H}_{29} \mathrm{~N}_{8} \mathrm{O}_{4} \mathrm{~S}_{2}[\mathrm{M}+\mathrm{H}]^{+}$545.17, found 544.79.

6-((4-(4-acetylpiperazin-1-yl)-2-methoxyphenyl)amino)-2,4,9-trimethyl-4,9-dihydro-10Hpyrimido[5,4-b]thiazolo[5,4-e][1,4]diazepin-10-one (15, BJG-04-064-1)<smiles>COc1cc(N2CCN(C(C)=O)CC2)ccc1Nc1ncc2c(n1)N(C)C(=O)c1nc(C)sc1N2C</smiles>

General Procedure E was run on 0.06 mmol scale using 1-(4-(4-amino-3methoxyphenyl)piperazin-1-yl)ethan-1-one (23.2 mg, $0.093 \mathrm{mmol}, 1.5$ equiv). The reaction mixture was purified by reverse-phase prep HPLC (100-50\% $\left.\mathrm{H}_{2} \mathrm{O} / \mathrm{MeCN}, 20 \mathrm{~mL} / \mathrm{min}, 45 \mathrm{~min}\right)$. Lyophilization from $\mathrm{H}_{2} \mathrm{O} / \mathrm{MeCN}$ provided the title compound as a white powder (10.7 mg TFA salt).

${ }^{1} \mathrm{H}$ NMR $\left(500 \mathrm{MHz}, \mathrm{DMSO}-d_{6}\right) \delta 8.31(\mathrm{~s}, 1 \mathrm{H}), 8.15(\mathrm{~s}, 1 \mathrm{H}), 7.69(\mathrm{~d}, J=8.7 \mathrm{~Hz}, 1 \mathrm{H}), 6.69(\mathrm{~d}, J=$ $2.6 \mathrm{~Hz}, 1 \mathrm{H}), 6.53(\mathrm{dd}, J=8.8,2.6 \mathrm{~Hz}, 1 \mathrm{H}), 3.81(\mathrm{~s}, 3 \mathrm{H}), 3.29(\mathrm{~s}, 3 \mathrm{H}), 3.26(\mathrm{~s}, 3 \mathrm{H}), 3.15(\mathrm{t}, J=5.2$ $\mathrm{Hz}, 2 \mathrm{H}), 3.09$ (t, J = 5.3 Hz, 2H), $2.54(\mathrm{~s}, 4 \mathrm{H}), 2.53$ (s, 3H), 2.05 (s, 3H). LRMS (ESI) calculated for $\mathrm{C}_{24} \mathrm{H}_{29} \mathrm{~N}_{8} \mathrm{O}_{3} \mathrm{~S}[\mathrm{M}+\mathrm{H}]^{+} 509.21$, found 509.16. 
6-((2-methoxy-5-methyl-4-(4-methylpiperazin-1-yl)phenyl)amino)-2,4,9-trimethyl-4,9dihydro-10H-pyrimido[5,4-b]thiazolo[5,4-e][1,4]diazepin-10-one (16, BJG-03-187-1)<smiles>COc1cc(N2CCN(C)CC2)c(C)cc1Nc1ncc2c(n1)N(C)C(=O)c1nc(C)sc1N2C</smiles>

General Procedure E was run on 0.05 mmol scale using 2-methoxy-5-methyl-4-(4methylpiperazin-1-yl)aniline (16.4 mg, $0.07 \mathrm{mmol}, 1.4$ equiv). The reaction mixture was purified by reverse-phase prep HPLC (100-45\% $\left.\mathrm{H}_{2} \mathrm{O} / \mathrm{MeCN}, 20 \mathrm{~mL} / \mathrm{min}, 45 \mathrm{~min}\right)$. Lyophilization from $\mathrm{H}_{2} \mathrm{O} / \mathrm{MeCN}$ provided the title compound as a light yellow powder (15.2 mg TFA salt).

${ }^{1} \mathrm{H}$ NMR (500 MHz, DMSO-d $) \delta 8.34(\mathrm{~s}, 1 \mathrm{H}), 8.07$ (s, 1H), $7.74(\mathrm{~s}, 1 \mathrm{H}), 6.71(\mathrm{~s}, 1 \mathrm{H}), 3.80(\mathrm{~s}$, $3 \mathrm{H}), 3.27(\mathrm{~s}, 3 \mathrm{H}), 2.86(\mathrm{t}, J=4.8 \mathrm{~Hz}, 4 \mathrm{H}), 2.53(\mathrm{~s}, 3 \mathrm{H}), 2.28(\mathrm{~s}, 3 \mathrm{H}), 2.18(\mathrm{~s}, 3 \mathrm{H})$. LRMS (ESI) calculated for $\mathrm{C}_{24} \mathrm{H}_{31} \mathrm{~N}_{8} \mathrm{O}_{2} \mathrm{~S}[\mathrm{M}+\mathrm{H}]^{+} 495.23$, found 494.88 .

6-((2-methoxy-4-(1-methylpiperidin-4-yl)phenyl)amino)-2,4,9-trimethyl-4,9-dihydro-10Hpyrimido[5,4-b]thiazolo[5,4-e][1,4]diazepin-10-one (17, BJG-04-015-1)<smiles>COc1cc(C2CCN(C)CC2)ccc1Nc1ncc2c(n1)N(C)C(=O)c1nc(C)sc1N2C</smiles>

General Procedure E was run on $0.06 \mathrm{mmol}$ scale using 2-methoxy-4-(1-methylpiperidin-4yl)aniline (18.3 mg, $0.083 \mathrm{mmol}, 1.4$ equiv). The reaction mixture was purified by reverse-phase 
prep HPLC (100-40\% $\left.\mathrm{H}_{2} \mathrm{O} / \mathrm{MeCN}, 20 \mathrm{~mL} / \mathrm{min}, 45 \mathrm{~min}\right)$. Lyophilization from $\mathrm{H}_{2} \mathrm{O} / \mathrm{MeCN}$ provided the title compound as a white powder ( $8.7 \mathrm{mg}$ TFA salt).

${ }^{1} \mathrm{H}$ NMR $\left(500 \mathrm{MHz}, \mathrm{DMSO}-d_{6}\right) \delta 9.47(\mathrm{~s}, 1 \mathrm{H}), 8.35(\mathrm{~s}, 1 \mathrm{H}), 8.16(\mathrm{~s}, 1 \mathrm{H}), 7.96(\mathrm{~d}, J=8.2 \mathrm{~Hz}, 1 \mathrm{H})$, $6.90(\mathrm{~d}, J=1.9 \mathrm{~Hz}, 1 \mathrm{H}), 6.81(\mathrm{dd}, J=8.3,1.9 \mathrm{~Hz}, 1 \mathrm{H}), 3.84(\mathrm{~s}, 3 \mathrm{H}), 3.33(\mathrm{~s}, 3 \mathrm{H}), 3.27(\mathrm{~s}, 3 \mathrm{H})$, $3.12-3.00(\mathrm{~m}, 2 \mathrm{H}), 2.81$ (s, 3H), $2.53(\mathrm{~s}, 3 \mathrm{H}), 2.03(\mathrm{~d}, J=13.9 \mathrm{~Hz}, 2 \mathrm{H}), 1.89-1.77(\mathrm{~m}, 2 \mathrm{H})$. LRMS (ESI) calculated for $\mathrm{C}_{24} \mathrm{H}_{30} \mathrm{~N}_{7} \mathrm{O}_{2} \mathrm{~S}[\mathrm{M}+\mathrm{H}]^{+} 480.22$, found 479.98 .

\section{6-((2-ethoxy-4-(4-methylpiperazin-1-yl)phenyl)amino)-2,4,9-trimethyl-4,9-dihydro-10H- pyrimido[5,4-b]thiazolo[5,4-e][1,4]diazepin-10-one (18, BJG-03-179-1)}

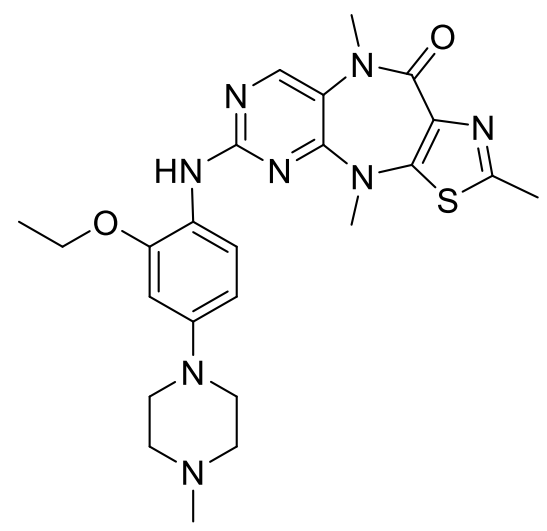

General Procedure E was run on $0.05 \mathrm{mmol}$ scale using 2-ethoxy-4-(4-methylpiperazin-1yl)aniline ( $18.3 \mathrm{mg}, 0.08 \mathrm{mmol}, 1.6$ equiv). The reaction mixture was purified by reverse-phase prep HPLC (100-50\% $\left.\mathrm{H}_{2} \mathrm{O} / \mathrm{MeCN}, 20 \mathrm{~mL} / \mathrm{min}, 45 \mathrm{~min}\right)$. Lyophilization from $\mathrm{H}_{2} \mathrm{O} / \mathrm{MeCN}$ provided the title compound as a yellow oil (5.5 $\mathrm{mg}$ free base).

${ }^{1} \mathrm{H}$ NMR (500 MHz, DMSO-d6) $\delta 8.31(\mathrm{~s}, 1 \mathrm{H}), 8.00(\mathrm{~s}, 1 \mathrm{H}), 7.70(\mathrm{~d}, \mathrm{~J}=8.8 \mathrm{~Hz}, 1 \mathrm{H}), 6.61(\mathrm{~d}, \mathrm{~J}=$ $2.6 \mathrm{~Hz}, 1 \mathrm{H}), 6.48(\mathrm{dd}, \mathrm{J}=8.8,2.6 \mathrm{~Hz}, 1 \mathrm{H}), 4.07(\mathrm{q}, \mathrm{J}=7.0 \mathrm{~Hz}, 2 \mathrm{H}), 3.29(\mathrm{~s}, 3 \mathrm{H}), 3.27(\mathrm{~s}, 3 \mathrm{H})$, $3.10(\mathrm{t}, \mathrm{J}=5.0 \mathrm{~Hz}, 4 \mathrm{H}), 2.53$ (s, 3H), 2.45 (t, J = 5.0 Hz, 4H), 2.22 (s, 3H), 1.29 (t, J = 7.0 Hz, 3H). LRMS (ESI) calculated for $\mathrm{C}_{24} \mathrm{H}_{31} \mathrm{~N}_{8} \mathrm{O}_{2} \mathrm{~S}[\mathrm{M}+\mathrm{H}]^{+} 495.23$, found 494.98 . 
6-((2-(2-hydroxyethoxy)-4-(4-methylpiperazin-1-yl)phenyl)amino)-2,4,9-trimethyl-4,9dihydro-10H-pyrimido[5,4-b]thiazolo[5,4-e][1,4]diazepin-10-one (19, BJG-03-179-2)<smiles>Cc1nc2c(s1)N(C)c1nc(Nc3ccc(N4CCN(C)CC4)cc3OCCO)ncc1N(C)C2=O</smiles>

General Procedure E was run on 0.05 mmol scale using 2-(2-amino-5-(4-methylpiperazin-1yl)phenoxy)ethan-1-ol (20.2 mg, $0.08 \mathrm{mmol}, 1.6$ equiv). The reaction mixture was purified by reverse-phase prep HPLC (100-50\% $\left.\mathrm{H}_{2} \mathrm{O} / \mathrm{MeCN}, 20 \mathrm{~mL} / \mathrm{min}, 45 \mathrm{~min}\right)$. Lyophilization from $\mathrm{H}_{2} \mathrm{O} / \mathrm{MeCN}$ provided the title compound as a light pink powder (7.0 $\mathrm{mg}$ free base).

${ }^{1} \mathrm{H}$ NMR (500 MHz, DMSO-d6) $\delta 9.64(\mathrm{~s}, 1 \mathrm{H}), 8.42(\mathrm{~s}, 1 \mathrm{H}), 8.35$ (s, 1H), $7.91(\mathrm{~d}, \mathrm{~J}=8.8 \mathrm{~Hz}, 1 \mathrm{H})$, $6.70(\mathrm{~d}, \mathrm{~J}=2.6 \mathrm{~Hz}, 1 \mathrm{H}), 6.57(\mathrm{dd}, \mathrm{J}=8.8,2.6 \mathrm{~Hz}, 1 \mathrm{H}), 5.11(\mathrm{~s}, 1 \mathrm{H}), 4.03(\mathrm{q}, \mathrm{J}=4.5 \mathrm{~Hz}, 2 \mathrm{H}), 3.81$ $(\mathrm{d}, \mathrm{J}=12.9 \mathrm{~Hz}, 2 \mathrm{H}), 3.71(\mathrm{t}, \mathrm{J}=4.7 \mathrm{~Hz}, 2 \mathrm{H}), 3.51(\mathrm{~s}, 3 \mathrm{H}), 3.28(\mathrm{~s}, 3 \mathrm{H}), 3.21-3.09(\mathrm{~m}, 3 \mathrm{H}), 2.95$ - $2.88(\mathrm{~m}, 2 \mathrm{H}), 2.87(\mathrm{~s}, 3 \mathrm{H}), 2.54(\mathrm{~s}, 3 \mathrm{H})$. LRMS (ESI) calculated for $\mathrm{C}_{24} \mathrm{H}_{31} \mathrm{~N}_{8} \mathrm{O}_{3} \mathrm{~S}[\mathrm{M}+\mathrm{H}]^{+}$ 511.22 , found 510.68 .

N-methyl-4-((2,4,9-trimethyl-10-oxo-9,10-dihydro-4H-pyrimido[5,4-b]thiazolo[5,4e][1,4]diazepin-6-yl)amino)benzamide (20, BJG-03-184-1)<smiles>CNC(=O)c1ccc(Nc2ncc3c(n2)N(C)c2sc(C)nc2C(=O)N3C)cc1</smiles> 
General Procedure E was run on $0.05 \mathrm{mmol}$ scale using 4-amino-N-methylbenzamide (13.0 mg, $0.086 \mathrm{mmol}, 1.7$ equiv). The reaction mixture was purified by reverse-phase prep HPLC (100-50\% $\left.\mathrm{H}_{2} \mathrm{O} / \mathrm{MeCN}, 20 \mathrm{~mL} / \mathrm{min}, 45 \mathrm{~min}\right)$. Further purification by prep TLC (10\% $\left.\mathrm{MeOH} / 2 \% \mathrm{NEt}_{3} / \mathrm{DCM}\right)$ and lyophilization from $\mathrm{H}_{2} \mathrm{O} / \mathrm{MeCN}$ provided the title compound as a white powder $(6.6 \mathrm{mg}$ free base).

${ }^{1} \mathrm{H}$ NMR (500 MHz, DMSO-d6) $\delta 9.97(\mathrm{~s}, 1 \mathrm{H}), 8.46$ (s, 1H), 8.24 (q, J=4.2 Hz, 1H), 7.78 (s, 4H), $3.40(\mathrm{~s}, 3 \mathrm{H}), 3.30(\mathrm{~s}, 3 \mathrm{H}), 2.76(\mathrm{~d}, J=4.4 \mathrm{~Hz}, 3 \mathrm{H}), 2.54(\mathrm{~s}, 3 \mathrm{H})$. LRMS (ESI) calculated for $\mathrm{C}_{19} \mathrm{H}_{20} \mathrm{~N}_{7} \mathrm{O}_{2} \mathrm{~S}[\mathrm{M}+\mathrm{H}]^{+} 410.14$, found 409.87 .

\section{3-methoxy-N-methyl-4-((2,4,9-trimethyl-10-oxo-9,10-dihydro-4H-pyrimido[5,4-} b]thiazolo[5,4-e][1,4]diazepin-6-yl)amino)benzamide (21, BJG-03-184-2)<smiles>CNC(=O)c1ccc(Nc2ncc3c(n2)N(C)C(=O)c2nc(C)sc2N3C)c(OC)c1</smiles>

General Procedure E was run on $0.05 \mathrm{mmol}$ scale using 4-amino-3-methoxy-N-methylbenzamide (12.8 mg, $0.07 \mathrm{mmol}, 1.4$ equiv). The reaction mixture was purified by reverse-phase prep HPLC (100-50\% $\left.\mathrm{H}_{2} \mathrm{O} / \mathrm{MeCN}, 20 \mathrm{~mL} / \mathrm{min}, 45 \mathrm{~min}\right)$. Further purification by prep TLC (10\% MeOH/2\% $\mathrm{NEt}_{3} / \mathrm{DCM}$ ) and lyophilization from $\mathrm{H}_{2} \mathrm{O} / \mathrm{MeCN}$ provided the title compound as a tan powder (5.4 mg free base).

${ }^{1} \mathrm{H}$ NMR (500 MHz, DMSO-d6) $\delta 8.44(\mathrm{~s}, 1 \mathrm{H}), 8.36(\mathrm{q}, J=4.5 \mathrm{~Hz}, 1 \mathrm{H}), 8.28-8.21(\mathrm{~m}, 2 \mathrm{H}), 7.52$ - 7.47 (m, 2H), 3.92 (s, 3H), 3.37 (s, 3H), 3.29 (s, 3H), 2.78 (d, J = 4.4 Hz, 3H), 2.54 (s, 3H). LRMS (ESI) calculated for $\mathrm{C}_{20} \mathrm{H}_{22} \mathrm{~N}_{7} \mathrm{O}_{3} \mathrm{~S}[\mathrm{M}+\mathrm{H}]^{+} 440.15$, found 439.98 . 


\section{2,4,9-trimethyl-6-((2-oxo-1,2,3,4-tetrahydroquinolin-6-yl)amino)-4,9-dihydro-10H-}

pyrimido[5,4-b]thiazolo[5,4-e][1,4]diazepin-10-one (22, BJG-04-013)<smiles>Cc1nc2c(s1)N(C)c1nc(Nc3ccc4c(c3)CCC(=O)N4)ncc1N(C)C2=O</smiles>

A solution of 6-chloro-2,4,9-trimethyl-4,9-dihydro-10H-pyrimido[5,4-b]thiazolo[5,4e][1,4]diazepin-10-one 10 (17.6 mg, $0.060 \mathrm{mmol}, 1.0$ equiv) and 6-amino-3,4-dihydroquinolin2(1H)-one (34.5 mg, $0.210 \mathrm{mmol}, 3.5$ equiv) in $4 \mathrm{M} \mathrm{HCl} /$ dioxane $(0.60 \mathrm{~mL})$ was stirred at $100{ }^{\circ} \mathrm{C}$ for 3 days. The reaction mixture was diluted with DCM and filtered through Celite, washing with $10 \mathrm{~mL} \mathrm{MeOH}$, and then purified by reverse-phase prep HPLC (100-50\% $\mathrm{H}_{2} \mathrm{O} / \mathrm{MeCN}, 20 \mathrm{~mL} / \mathrm{min}$, $45 \mathrm{~min})$. Further purification by prep TLC (10\% $\left.\mathrm{MeOH} / 2 \% \mathrm{NEt}_{3} / \mathrm{DCM}\right)$ and lyophilization from $\mathrm{H}_{2} \mathrm{O} / \mathrm{MeCN}$ provided the title compound as a beige powder (1.6 mg free base).

LRMS (ESI) calculated for $\mathrm{C}_{20} \mathrm{H}_{20} \mathrm{~N}_{7} \mathrm{O}_{2} \mathrm{~S}[\mathrm{M}+\mathrm{H}]^{+}$422.14, found 421.87 .

6-((6-methoxy-2-methyl-1,2,3,4-tetrahydroisoquinolin-7-yl)amino)-2,4,9-trimethyl-4,9dihydro-10H-pyrimido[5,4-b]thiazolo[5,4-e][1,4]diazepin-10-one (23, BJG-03-187-2)<smiles>COc1cc2c(cc1Nc1ncc3c(n1)N(C)C(=O)c1nc(C)sc1N3C)CN(C)CC2</smiles>

General Procedure E was run on $0.05 \mathrm{mmol}$ scale using 6-methoxy-2-methyl-1,2,3,4tetrahydroisoquinolin-7-amine $(12.7 \mathrm{mg}, 0.065 \mathrm{mmol}, 1.3$ equiv). The reaction mixture was purified by reverse-phase prep HPLC (100-45\% $\mathrm{H}_{2} \mathrm{O} / \mathrm{MeCN}, 20 \mathrm{~mL} / \mathrm{min}, 45 \mathrm{~min}$ ). Lyophilization from $\mathrm{H}_{2} \mathrm{O} / \mathrm{MeCN}$ provided the title compound as a yellow powder (11.3 mg TFA salt). 
${ }^{1} \mathrm{H}$ NMR (500 MHz, DMSO-d $) \delta 8.37$ (s, 1H), $8.08(\mathrm{~s}, 1 \mathrm{H}), 7.74(\mathrm{~s}, 1 \mathrm{H}), 6.77(\mathrm{~s}, 1 \mathrm{H}), 3.80$ (s, $3 \mathrm{H}), 3.49(\mathrm{~s}, 2 \mathrm{H}), 3.28(\mathrm{~s}, 3 \mathrm{H}), 2.80(\mathrm{t}, J=6.0 \mathrm{~Hz}, 2 \mathrm{H}), 2.69-2.60(\mathrm{~m}, 2 \mathrm{H}), 2.54(\mathrm{~s}, 3 \mathrm{H}), 2.38(\mathrm{~s}$, $3 \mathrm{H})$. LRMS (ESI) calculated for $\mathrm{C}_{22} \mathrm{H}_{26} \mathrm{~N}_{7} \mathrm{O}_{2} \mathrm{~S}[\mathrm{M}+\mathrm{H}]^{+} 452.19$, found 451.88 .

6-((4-(4-(2-hydroxyethyl)piperazin-1-yl)-2-methoxyphenyl)amino)-2,4,9-trimethyl-4,9dihydro-10H-pyrimido[5,4-b]thiazolo[5,4-e][1,4]diazepin-10-one (24, BJG-04-015-2)<smiles>COc1cc(N2CCN(CCO)CC2)ccc1Nc1ncc2c(n1)N(C)c1sc(C)nc1C(=O)N2C</smiles>

General Procedure E was run on 0.06 mmol scale using 2-(4-(4-amino-3methoxyphenyl)piperazin-1-yl)ethan-1-ol (20.6 mg, $0.082 \mathrm{mmol}, 1.4$ equiv). The reaction mixture was purified by reverse-phase prep HPLC (100-40\% $\left.\mathrm{H}_{2} \mathrm{O} / \mathrm{MeCN}, 20 \mathrm{~mL} / \mathrm{min}, 45 \mathrm{~min}\right)$. Lyophilization from $\mathrm{H}_{2} \mathrm{O} / \mathrm{MeCN}$ provided the title compound as a brown powder (15.6 mg TFA salt).

${ }^{1} \mathrm{H}$ NMR (500 MHz, DMSO-d6) $\delta 9.68(\mathrm{~s}, 1 \mathrm{H}), 8.31(\mathrm{~s}, 1 \mathrm{H}), 8.13(\mathrm{~s}, 1 \mathrm{H}), 7.72(\mathrm{~d}, J=8.7 \mathrm{~Hz}, 1 \mathrm{H})$, $6.70(\mathrm{~d}, J=2.6 \mathrm{~Hz}, 1 \mathrm{H}), 6.55(\mathrm{dd}, J=8.8,2.6 \mathrm{~Hz}, 1 \mathrm{H}), 5.42(\mathrm{~s}, 1 \mathrm{H}), 3.82(\mathrm{~s}, 3 \mathrm{H}), 3.78(\mathrm{t}, J=5.4$ $\mathrm{Hz}, 2 \mathrm{H}), 3.66-3.52(\mathrm{~m}, 2 \mathrm{H}), 3.30(\mathrm{~s}, 3 \mathrm{H}), 3.27$ (s, 3H), $3.21(\mathrm{~s}, 2 \mathrm{H}), 3.05$ (d, J = 13.3 Hz, 2H), $2.53(\mathrm{~s}, 3 \mathrm{H})$. LRMS (ESI) calculated for $\mathrm{C}_{24} \mathrm{H}_{31} \mathrm{~N}_{8} \mathrm{O}_{3} \mathrm{~S}[\mathrm{M}+\mathrm{H}]^{+}$511.22, found 510.88 . 
6-((4-(4-(2-(dimethylamino)ethyl)piperazin-1-yl)-2-methoxyphenyl)amino)-2,4,9-trimethyl4,9-dihydro-10H-pyrimido[5,4-b] thiazolo[5,4-e][1,4]diazepin-10-one (25, BJG-04-064-2)<smiles>COc1cc(N2CCN(CCN(C)C)CC2)ccc1Nc1ncc2c(n1)N(C)c1sc(C)nc1C(=O)N2C</smiles>

General Procedure E was run on 0.06 mmol scale using 4-(4-(2-(dimethylamino)ethyl)piperazin1-yl)-2-methoxyaniline ( $25.4 \mathrm{mg}, 0.091 \mathrm{mmol}, 1.5$ equiv). The reaction mixture was purified by reverse-phase prep HPLC (100-50\% $\left.\mathrm{H}_{2} \mathrm{O} / \mathrm{MeCN}, 20 \mathrm{~mL} / \mathrm{min}, 45 \mathrm{~min}\right)$. Further purification by prep TLC (10\% $\left.\mathrm{MeOH} / 2 \% \mathrm{NEt}_{3} / \mathrm{DCM}\right)$ and lyophilization from $\mathrm{H}_{2} \mathrm{O} / \mathrm{MeCN}$ provided the title compound as a white powder (14.7 $\mathrm{mg}$ free base).

${ }^{1} \mathrm{H}$ NMR $\left(500 \mathrm{MHz}, \mathrm{DMSO}-d_{6}\right) \delta 9.10(\mathrm{~s}, 1 \mathrm{H}), 8.30(\mathrm{~s}, 1 \mathrm{H}), 8.12(\mathrm{~s}, 1 \mathrm{H}), 7.69(\mathrm{~d}, J=8.7 \mathrm{~Hz}, 1 \mathrm{H})$, $6.67(\mathrm{~d}, J=2.5 \mathrm{~Hz}, 1 \mathrm{H}), 6.53(\mathrm{dd}, J=8.8,2.5 \mathrm{~Hz}, 1 \mathrm{H}), 3.81$ (s, 3H), 3.29 (s, 3H), 3.27 (s, 3H), $2.83(\mathrm{~s}, 6 \mathrm{H}), 2.53(\mathrm{~s}, 3 \mathrm{H})$. LRMS (ESI) calculated for $\mathrm{C}_{26} \mathrm{H}_{36} \mathrm{~N}_{9} \mathrm{O}_{2} \mathrm{~S}[\mathrm{M}+\mathrm{H}]^{+}$538.27, found 538.23. 
2,4,9-trimethyl-6-((5-(4-methylpiperazin-1-yl)pyridin-2-yl)amino)-4,9-dihydro-10Hpyrimido[5,4-b]thiazolo[5,4-e][1,4]diazepin-10-one (26, BJG-03-204)<smiles>Cc1nc2c(s1)N(C)c1nc(Nc3ccc(N4CCN(C)CC4)cn3)ncc1N(C)C2=O</smiles>

General Procedure E was run on $0.08 \mathrm{mmol}$ scale using 5-(4-methylpiperazin-1-yl)pyridin-2amine ( $21.6 \mathrm{mg}, 0.11 \mathrm{mmol}, 1.4$ equiv). The reaction mixture was purified by reverse-phase prep HPLC (100-50\% $\left.\mathrm{H}_{2} \mathrm{O} / \mathrm{MeCN}, 20 \mathrm{~mL} / \mathrm{min}, 45 \mathrm{~min}\right)$. Further purification by prep TLC (10\% $\mathrm{MeOH} / 2 \% \mathrm{NEt}_{3} / \mathrm{DCM}$ ) and lyophilization from $\mathrm{H}_{2} \mathrm{O} / \mathrm{MeCN}$ provided the title compound as a beige powder (1.7 $\mathrm{mg}$ free base).

LRMS (ESI) calculated for $\mathrm{C}_{21} \mathrm{H}_{26} \mathrm{~N}_{9} \mathrm{OS}[\mathrm{M}+\mathrm{H}]^{+} 452.20$, found 451.88 .

\section{2,4,9-trimethyl-6-((1-methyl-1H-pyrazol-4-yl)amino)-4,9-dihydro-10H-pyrimido[5,4- b] thiazolo[5,4-e][1,4]diazepin-10-one (27, BJG-04-006-1)}<smiles>Cc1nc2c(s1)N(C)c1nc(Nc3cnn(C)c3)ncc1N(C)C2=O</smiles>

General Procedure E was run on $0.06 \mathrm{mmol}$ scale using 1-methyl-1H-pyrazol-4-amine (12.6 mg, $0.13 \mathrm{mmol}, 2.15$ equiv). The reaction mixture was purified by reverse-phase prep HPLC (100-50\% $\mathrm{H}_{2} \mathrm{O} / \mathrm{MeCN}, 20 \mathrm{~mL} / \mathrm{min}, 45 \mathrm{~min}$ ). Lyophilization from $\mathrm{H}_{2} \mathrm{O} / \mathrm{MeCN}$ provided the title compound as a white powder (5.3 $\mathrm{mg}$ TFA salt). 
${ }^{1} \mathrm{H}$ NMR (500 MHz, DMSO-d $\left.d_{6}\right) \delta 9.58(\mathrm{~s}, 1 \mathrm{H}), 8.35$ (s, 1H), 7.82 (s, 1H), $7.48(\mathrm{~s}, 1 \mathrm{H}), 3.81$ (s, $3 \mathrm{H}), 3.27(\mathrm{~s}, 3 \mathrm{H}), 3.17(\mathrm{~s}, 3 \mathrm{H}), 2.54(\mathrm{~s}, 3 \mathrm{H})$. LRMS (ESI) calculated for $\mathrm{C}_{15} \mathrm{H}_{17} \mathrm{~N}_{8} \mathrm{OS}[\mathrm{M}+\mathrm{H}]^{+}$ 357.12 , found 356.97 .

6-((2-methoxy-4-(4-(4-methylpiperazin-1-yl)piperidine-1-carbonyl)phenyl)amino)-2,4,9trimethyl-4,9-dihydro-10H-pyrimido[5,4-b]thiazolo[5,4-e][1,4]diazepin-10-one (28, BJG-4005)<smiles>COc1cc(C(=O)N2CCC(N3CCN(C)CC3)CC2)ccc1Nc1ncc2c(n1)N(C)c1sc(C)nc1C(=O)N2C</smiles>

General Procedure E was run on 0.06 mmol scale using (4-amino-3-methoxyphenyl)-(4-(4methylpiperazin-1-yl)piperidin-1-yl)methanone (38.1 mg, $0.115 \mathrm{mmol}, 1.9$ equiv). The reaction mixture was purified by reverse-phase prep HPLC (100-50\% $\left.\mathrm{H}_{2} \mathrm{O} / \mathrm{MeCN}, 20 \mathrm{~mL} / \mathrm{min}, 45 \mathrm{~min}\right)$. Lyophilization from $\mathrm{H}_{2} \mathrm{O} / \mathrm{MeCN}$ provided the title compound as a white powder (6.4 mg TFA salt).

${ }^{1} \mathrm{H}$ NMR (500 MHz, DMSO-d6) $\delta 8.42(\mathrm{~s}, 1 \mathrm{H}), 8.26(\mathrm{~s}, 1 \mathrm{H}), 8.18(\mathrm{~d}, J=8.2 \mathrm{~Hz}, 1 \mathrm{H}), 7.01(\mathrm{dd}, J$ $=8.2,1.8 \mathrm{~Hz}, 1 \mathrm{H}), 3.89(\mathrm{~s}, 3 \mathrm{H}), 3.36(\mathrm{~s}, 3 \mathrm{H}), 3.29(\mathrm{~s}, 3 \mathrm{H}), 2.75(\mathrm{~s}, 3 \mathrm{H}), 2.54(\mathrm{~s}, 3 \mathrm{H}), 1.96-1.78$ $(\mathrm{m}, 2 \mathrm{H}), 1.54-1.38(\mathrm{~m}, 2 \mathrm{H})$ (Piperazine resonances obscured under water peak). LRMS (ESI) calculated for $\mathrm{C}_{29} \mathrm{H}_{38} \mathrm{~N}_{9} \mathrm{O}_{3} \mathrm{~S}[\mathrm{M}+\mathrm{H}]^{+}$592.28, found 591.89. 


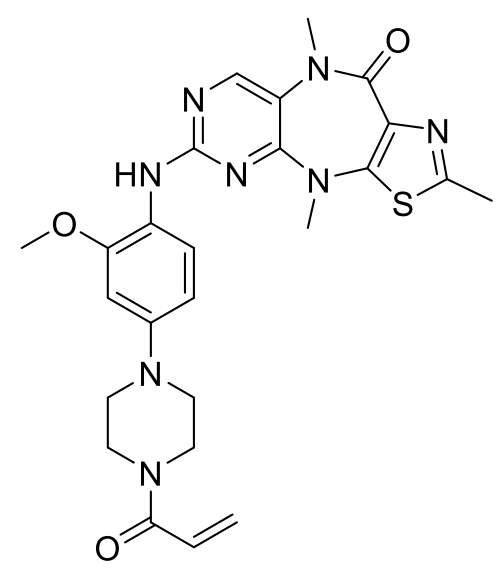

To a suspension of 12 (26.1 mg, $0.045 \mathrm{mmol}, 1.0$ equiv) in DCM (1.0 mL) at room temperature were added triethylamine $(30.00 \mu \mathrm{L}, 0.215 \mathrm{mmol}, 4.8$ equiv $)$ and then acryloyl chloride $(6.00 \mu \mathrm{L}$, $0.074 \mathrm{mmol}, 1.6$ equiv). The reaction was stirred for 2 hours, at which point UPLC-MS analysis showed full conversion of 12 . The mixture was quenched with water $(5 \mathrm{~mL})$ and extracted with DCM $(5 \times 5 \mathrm{~mL})$. The combined organic layers were washed with saturated aqueous $\mathrm{NaHCO}_{3}$ and then brine, dried over $\mathrm{MgSO}_{4}$, filtered and concentrated. Reverse-phase prep HPLC purification (100-25\% $\mathrm{H}_{2} \mathrm{O} / \mathrm{MeCN}, 20 \mathrm{~mL} / \mathrm{min}, 45 \mathrm{~min}$ ) and lyophilization from $\mathrm{H}_{2} \mathrm{O} / \mathrm{MeCN}$ provided the title compound as a yellow oil (2.8 mg TFA salt).

${ }^{1} \mathrm{H}$ NMR (500 MHz, DMSO-d $) \delta 8.31(\mathrm{~s}, 1 \mathrm{H}), 8.11(\mathrm{~s}, 1 \mathrm{H}), 7.69(\mathrm{~d}, J=8.7 \mathrm{~Hz}, 1 \mathrm{H}), 6.86(\mathrm{dd}, J$ $=16.7,10.5 \mathrm{~Hz}, 1 \mathrm{H}), 6.68(\mathrm{~d}, J=2.6 \mathrm{~Hz}, 1 \mathrm{H}), 6.52(\mathrm{dd}, J=8.7,2.6 \mathrm{~Hz}, 1 \mathrm{H}), 6.14(\mathrm{dd}, J=16.7$, $2.5 \mathrm{~Hz}, 1 \mathrm{H}), 5.71(\mathrm{dd}, J=10.5,2.3 \mathrm{~Hz}, 1 \mathrm{H}), 3.81(\mathrm{~s}, 3 \mathrm{H}), 3.74-3.64(\mathrm{~m}, 4 \mathrm{H}), 3.29(\mathrm{~s}, 3 \mathrm{H}), 3.26$ $(\mathrm{s}, 3 \mathrm{H}), 3.16-3.08(\mathrm{~m}, 4 \mathrm{H}), 2.53(\mathrm{~s}, 3 \mathrm{H})$. LRMS (ESI) calculated for $\mathrm{C}_{25} \mathrm{H}_{29} \mathrm{~N}_{8} \mathrm{O}_{3} \mathrm{~S}[\mathrm{M}+\mathrm{H}]^{+}$ 521.21, found 520.88 . 
6-((2-methoxy-4-(4-(1-methylpiperidin-4-yl)piperazin-1-yl)phenyl)amino)-2,4,9-trimethyl4,9-dihydro-10H-pyrimido[5,4-b]thiazolo[5,4-e][1,4]diazepin-10-one (S-51, BJG-04-155)<smiles>COc1cc(N2CCN(C3CCN(C)CC3)CC2)ccc1Nc1ncc2c(n1)N(C)c1sc(C)nc1C(=O)N2C</smiles>

General Procedure E was run on 0.07 mmol scale using 2-methoxy-4-(4-(1-methylpiperidin-4yl)piperazin-1-yl)aniline (29.5 mg, $0.105 \mathrm{mmol}, 1.5$ equiv). The reaction mixture was purified by reverse-phase prep HPLC (100-50\% $\left.\mathrm{H}_{2} \mathrm{O} / \mathrm{MeCN}, 20 \mathrm{~mL} / \mathrm{min}, 45 \mathrm{~min}\right)$. Further purification by prep TLC (10\% MeOH/DCM) and lyophilization from $\mathrm{H}_{2} \mathrm{O} / \mathrm{MeCN}$ provided the title compound as a white powder (13.7 mg TFA salt).

${ }^{1} \mathrm{H}$ NMR $\left(500 \mathrm{MHz}, \mathrm{DMSO}-d_{6}\right) \delta 8.31(\mathrm{~s}, 1 \mathrm{H}), 8.11(\mathrm{~s}, 1 \mathrm{H}), 7.70(\mathrm{~d}, J=6.6 \mathrm{~Hz}, 1 \mathrm{H}), 6.68(\mathrm{~s}, 1 \mathrm{H})$, $6.53(\mathrm{~d}, J=8.6 \mathrm{~Hz}, 1 \mathrm{H}), 3.81(\mathrm{~s}, 3 \mathrm{H}), 3.62-3.48(\mathrm{~m}, 2 \mathrm{H}), 3.29(\mathrm{~s}, 3 \mathrm{H}), 3.27(\mathrm{~s}, 3 \mathrm{H}), 3.02-2.90(\mathrm{~m}$, $2 \mathrm{H}), 2.77(\mathrm{~s}, 3 \mathrm{H}), 2.53$ (s, 3H). LRMS (ESI) calculated for $\mathrm{C}_{28} \mathrm{H}_{38} \mathrm{~N}_{9} \mathrm{O}_{2} \mathrm{~S}[\mathrm{M}+\mathrm{H}]^{+} 564.29$, found 563.99 .

2,4,9-trimethyl-6-((4-(4-methylpiperazin-1-yl)-2-(2,2,2-trifluoroethoxy)phenyl)amino)-4,9dihydro-10H-pyrimido[5,4-b]thiazolo[5,4-e][1,4]diazepin-10-one (S-52, CRC-01-042)<smiles>Cc1nc2c(s1)N(C)c1nc(Nc3ccc(N4CCN(C)CC4)cc3OCC(F)(F)F)ncc1N(C)C2=O</smiles>

General Procedure E was run on $0.05 \mathrm{mmol}$ scale using 4-(4-methylpiperazin-1-yl)-2-(2,2,2trifluoroethoxy)aniline (20 mg, $0.069 \mathrm{mmol}, 1.4$ equiv). The reaction mixture was purified by reverse-phase prep HPLC (100-50\% $\left.\mathrm{H}_{2} \mathrm{O} / \mathrm{MeCN}, 20 \mathrm{~mL} / \mathrm{min}, 45 \mathrm{~min}\right)$. Further purification by 
prep TLC (10\% MeOH/DCM) and lyophilization from $\mathrm{H}_{2} \mathrm{O} / \mathrm{MeCN}$ provided the title compound as a white powder (2.6 mg TFA salt).

${ }^{1} \mathrm{H}$ NMR $\left(500 \mathrm{MHz}\right.$, DMSO- $\left.d_{6}\right) \delta 8.28(\mathrm{~s}, 1 \mathrm{H}), 8.22(\mathrm{~s}, 1 \mathrm{H}), 7.50(\mathrm{~d}, J=8.8 \mathrm{~Hz}, 1 \mathrm{H}), 6.74(\mathrm{~d}, J=$ $2.6 \mathrm{~Hz}, 1 \mathrm{H}), 6.58(\mathrm{dd}, J=8.8,2.6 \mathrm{~Hz}, 1 \mathrm{H}), 4.73$ (q, $J=8.9 \mathrm{~Hz}, 2 \mathrm{H}), 3.25(\mathrm{~s}, 6 \mathrm{H}), 3.13$ (t, $J=5.0$ $\mathrm{Hz}, 4 \mathrm{H}), 2.53(\mathrm{~s}, 3 \mathrm{H}), 2.45$ (t, $J=5.1 \mathrm{~Hz}, 4 \mathrm{H}), 2.22(\mathrm{~s}, 3 \mathrm{H}) .{ }^{19} \mathrm{~F}$ NMR $\left(471 \mathrm{MHz}\right.$, DMSO- $\left.d_{6}\right) \delta-$ $72.62\left(\mathrm{t}, J=8.9 \mathrm{~Hz}\right.$ ). LRMS (ESI) calculated for $\mathrm{C}_{24} \mathrm{H}_{28} \mathrm{~F}_{3} \mathrm{~N}_{8} \mathrm{O}_{2} \mathrm{~S}[\mathrm{M}+\mathrm{H}]^{+} 549.20$, found 548.89 .

\section{2,4,9-trimethyl-6-((4-(4-methylpiperazin-1-yl)-2-propoxyphenyl)amino)-4,9-dihydro-10H- pyrimido[5,4-b]thiazolo[5,4-e][1,4]diazepin-10-one (S-53, CRC-01-031)}

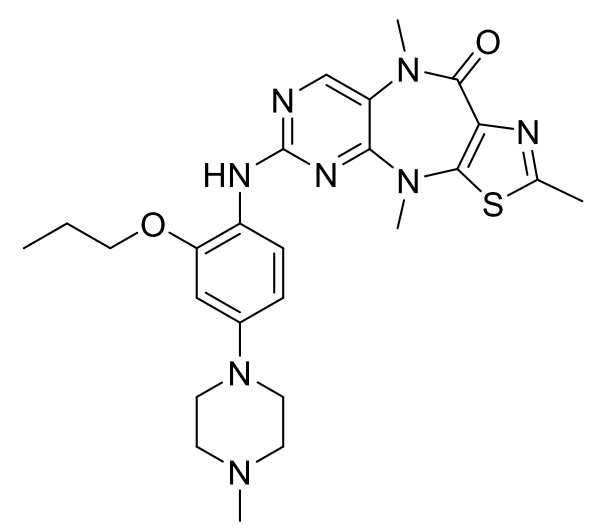

General Procedure E was run on 0.05 mmol scale using 4-(4-methylpiperazin-1-yl)-2propoxyaniline ( $17 \mathrm{mg}, 0.068 \mathrm{mmol}, 1.3$ equiv). The reaction mixture was purified by reversephase prep HPLC (100-50\% $\mathrm{H}_{2} \mathrm{O} / \mathrm{MeCN}, 20 \mathrm{~mL} / \mathrm{min}, 45 \mathrm{~min}$ ). Further purification by prep TLC $(10 \% \mathrm{MeOH} / \mathrm{DCM})$ and lyophilization from $\mathrm{H}_{2} \mathrm{O} / \mathrm{MeCN}$ provided the title compound as a white powder (6.8 mg TFA salt).

${ }^{1} \mathrm{H}$ NMR (500 MHz, DMSO- $\left.d_{6}\right) \delta 8.24(\mathrm{~s}, 1 \mathrm{H}), 7.96(\mathrm{~s}, 1 \mathrm{H}), 7.57(\mathrm{~d}, J=8.8 \mathrm{~Hz}, 1 \mathrm{H}), 6.55(\mathrm{~d}, J=$ $2.6 \mathrm{~Hz}, 1 \mathrm{H}), 6.41(\mathrm{dd}, J=8.8,2.6 \mathrm{~Hz}, 1 \mathrm{H}), 3.88$ (t, $J=6.5 \mathrm{~Hz}, 2 \mathrm{H}), 3.21$ (s, $3 \mathrm{H}), 3.19$ (s, 3H), 3.05 (s, 4H), 2.46 (s, 3H), 2.20 (br s, 2H), $1.67-1.56(\mathrm{~m}, 2 \mathrm{H}), 0.82$ (t, $J=7.4 \mathrm{~Hz}, 3 \mathrm{H})$. LRMS (ESI) calculated for $\mathrm{C}_{25} \mathrm{H}_{33} \mathrm{~N}_{8} \mathrm{O}_{2} \mathrm{~S}[\mathrm{M}+\mathrm{H}]^{+} 509.24$, found 508.98 . 
6-((2-methoxy-4-(4-(4-methylpiperazin-1-yl)piperidin-1-yl)phenyl)amino)-4-methyl-4,9-

dihydro-10H-pyrimido[5,4-b]thiazolo[5,4-e][1,4]diazepin-10-one (29, BJG-03-084)

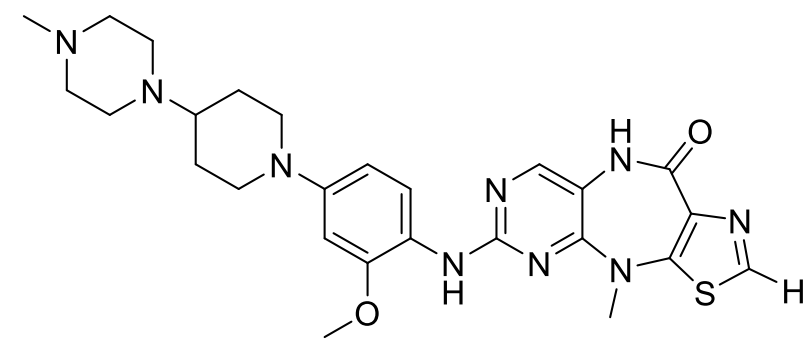

General Procedure E was run on $0.08 \mathrm{mmol}$ scale using 2-methoxy-4-(4-(4-methylpiperazin-1yl)piperidin-1-yl)aniline (34.6 mg, $0.11 \mathrm{mmol}, 1.4$ equiv). The reaction mixture was purified by reverse-phase prep HPLC (100-30\% $\left.\mathrm{H}_{2} \mathrm{O} / \mathrm{MeCN}, 20 \mathrm{~mL} / \mathrm{min}, 28 \mathrm{~min}\right)$. The material was further purified by prep TLC (10\% MeOH/DCM). Lyophilization from $\mathrm{H}_{2} \mathrm{O} / \mathrm{MeCN}$ provided the title compound as a dark gray powder (3.4 mg TFA salt).

${ }^{1} \mathrm{H}$ NMR (500 MHz, DMSO-d6) $\delta 9.62$ (s, 1H), 8.67 (s, 1H), 8.02 (s, 1H), 7.95 (s, 1H), 7.75 (d, $J$ $=8.7 \mathrm{~Hz}, 1 \mathrm{H}), 6.75(\mathrm{~s}, 1 \mathrm{H}), 6.61(\mathrm{~s}, 1 \mathrm{H}), 3.82(\mathrm{~s}, 3 \mathrm{H}), 3.80-3.74(\mathrm{~m}, 3 \mathrm{H}), 3.66-3.44(\mathrm{~m}, 5 \mathrm{H})$, $3.36(\mathrm{~s}, 3 \mathrm{H}), 2.81$ (br s, 7H), $2.08-2.00(\mathrm{~m}, 3 \mathrm{H}), 1.75-1.64(\mathrm{~m}, 3 \mathrm{H})$. LRMS (ESI) calculated for $\mathrm{C}_{26} \mathrm{H}_{34} \mathrm{~N}_{9} \mathrm{O}_{2} \mathrm{~S}[\mathrm{M}+\mathrm{H}]^{+}$536.26, found 536.38.

2-ethyl-6-((2-methoxy-4-(4-(4-methylpiperazin-1-yl)piperidin-1-yl)phenyl)amino)-4,9dimethyl-4,9-dihydro-10H-pyrimido[5,4-b]thiazolo[5,4-e][1,4]diazepin-10-one (30, CRC-01025)<smiles>CCc1nc2c(s1)N(C)c1nc(Nc3ccc(N4CCC(N5CCN(C)CC5)CC4)cc3OC)ncc1N(C)C2=O</smiles>

General Procedure E was run on $0.16 \mathrm{mmol}$ scale using 2-methoxy-4-(4-(4-methylpiperazin-1yl)piperidin-1-yl)aniline (70.9 mg, $0.23 \mathrm{mmol}, 1.5$ equiv). The reaction mixture was purified by reverse-phase prep HPLC (85-15\% $\left.\mathrm{H}_{2} \mathrm{O} / \mathrm{MeCN}, 40 \mathrm{~mL} / \mathrm{min}, 60 \mathrm{~min}\right)$. Further purification by prep 
TLC (10\% MeOH/DCM) and lyophilization from $\mathrm{H}_{2} \mathrm{O} / \mathrm{MeCN}$ provided the title compound as a light yellow powder (19.2 mg TFA salt).

${ }^{1} \mathrm{H}$ NMR (500 MHz, DMSO-d6) $\delta 8.31$ (s, 1H), 8.14 (s, 1H), 7.70 (d, J = 8.7 Hz, 1H), $6.71(\mathrm{~s}, 1 \mathrm{H})$, $6.58(\mathrm{~d}, \mathrm{~J}=8.6 \mathrm{~Hz}, 1 \mathrm{H}), 3.84-3.74(\mathrm{~m}, 9 \mathrm{H}), 3.31(\mathrm{~s}, 3 \mathrm{H}), 3.27(\mathrm{~s}, 3 \mathrm{H}), 2.86(\mathrm{q}, \mathrm{J}=7.5 \mathrm{~Hz}, 2 \mathrm{H})$, 2.78 (br s, 5H), $2.06-1.96(\mathrm{~m}, 2 \mathrm{H}), 1.65$ (d, J = $12.3 \mathrm{~Hz}, 2 \mathrm{H}), 1.23$ (t, J = 7.5 Hz, 3H). LRMS (ESI) calculated for $\mathrm{C}_{29} \mathrm{H}_{40} \mathrm{~N}_{9} \mathrm{O}_{2} \mathrm{~S}[\mathrm{M}+\mathrm{H}]^{+} 578.30$, found 577.98 .

2-isopropyl-6-((2-methoxy-4-(4-(4-methylpiperazin-1-yl)piperidin-1-yl)phenyl)amino)-4,9dimethyl-4,9-dihydro-10H-pyrimido[5,4-b]thiazolo[5,4-e][1,4]diazepin-10-one (31, CRC-01033)

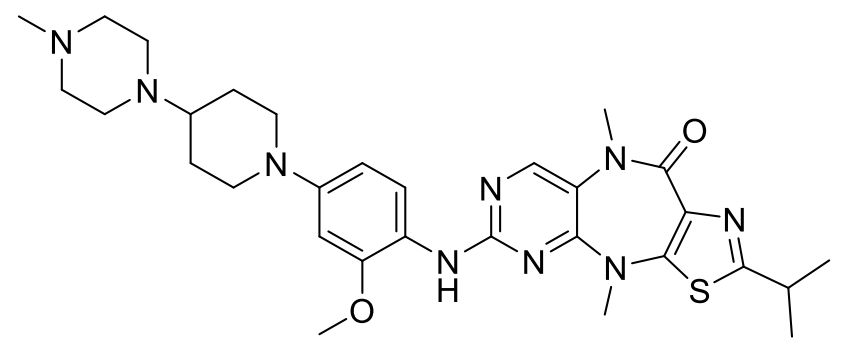

General Procedure E was run on $0.08 \mathrm{mmol}$ scale using 2-methoxy-4-(4-(4-methylpiperazin-1yl)piperidin-1-yl)aniline (31.6 mg, $0.104 \mathrm{mmol}, 1.3$ equiv). The reaction mixture was purified by reverse-phase prep HPLC (100-50\% $\left.\mathrm{H}_{2} \mathrm{O} / \mathrm{MeCN}, 20 \mathrm{~mL} / \mathrm{min}, 45 \mathrm{~min}\right)$. Further purification by prep TLC (10\% MeOH/DCM) and lyophilization from $\mathrm{H}_{2} \mathrm{O} / \mathrm{MeCN}$ provided the title compound as a white powder (8.5 mg TFA salt).

${ }^{1} \mathrm{H}$ NMR (500 MHz, DMSO-d $) \delta 8.29(\mathrm{~s}, 1 \mathrm{H}), 8.08(\mathrm{~s}, 1 \mathrm{H}), 7.64(\mathrm{~d}, J=8.7 \mathrm{~Hz}, 1 \mathrm{H}), 6.62(\mathrm{~s}, 1 \mathrm{H})$, $6.50(\mathrm{~d}, J=8.8 \mathrm{~Hz}, 1 \mathrm{H}), 3.80$ (s, 3H), 3.70 (s, 2H), 3.30 (s, 3H), 3.27 (s, 3H), 3.16 (p, J = 6.9 Hz, 1H), 3.10-2.89 (m, 4H), 2.82-2.71 (m, 2H), $2.66(\mathrm{t}, J=12.0 \mathrm{~Hz}, 2 \mathrm{H}), 1.95-1.74(\mathrm{~m}, 2 \mathrm{H}), 1.60-$ $1.44(\mathrm{~m}, 2 \mathrm{H}), 1.27(\mathrm{~d}, J=6.9 \mathrm{~Hz}, 6 \mathrm{H})$. LRMS (ESI) calculated for $\mathrm{C}_{30} \mathrm{H}_{42} \mathrm{~N}_{9} \mathrm{O}_{2} \mathrm{~S}[\mathrm{M}+\mathrm{H}]^{+} 592.32$, found 591.99. 
6-((2-methoxy-4-(4-(4-methylpiperazin-1-yl)piperidin-1-yl)phenyl)amino)-4,9-dimethyl-2phenyl-4,9-dihydro-10H-pyrimido[5,4-b]thiazolo[5,4-e][1,4]diazepin-10-one (32, BJG-04020)<smiles>COc1cc(N2CCC(N3CCN(C)CC3)CC2)ccc1Nc1ncc2c(n1)N(C)c1sc(-c3ccccc3)nc1C(=O)N2C</smiles>

General Procedure E was run on 0.065 mmol scale using 2-methoxy-4-(4-(4-methylpiperazin-1yl)piperidin-1-yl)aniline ( $27.8 \mathrm{mg}, 0.091 \mathrm{mmol}, 1.4$ equiv). The reaction mixture was purified by reverse-phase prep HPLC (100-50\% $\left.\mathrm{H}_{2} \mathrm{O} / \mathrm{MeCN}, 20 \mathrm{~mL} / \mathrm{min}, 45 \mathrm{~min}\right)$. Further purification by prep TLC (10\% MeOH/2\% $\left.\mathrm{NEt}_{3} / \mathrm{DCM}\right)$ and lyophilization from $\mathrm{H}_{2} \mathrm{O} / \mathrm{MeCN}$ provided the title compound as a yellow solid (8.1 $\mathrm{mg}$ free base).

${ }^{1} \mathrm{H}$ NMR $\left(500 \mathrm{MHz}, \mathrm{DMSO}-d_{6}\right) \delta 8.34(\mathrm{~s}, 1 \mathrm{H}), 8.14(\mathrm{~d}, J=1.4 \mathrm{~Hz}, 1 \mathrm{H}), 7.87-7.80(\mathrm{~m}, 2 \mathrm{H}), 7.64$ $(\mathrm{d}, J=8.7 \mathrm{~Hz}, 1 \mathrm{H}), 7.54-7.44(\mathrm{~m}, 3 \mathrm{H}), 6.63(\mathrm{~s}, 1 \mathrm{H}), 6.54-6.46(\mathrm{~m}, 1 \mathrm{H}), 3.80(\mathrm{~s}, 3 \mathrm{H}), 3.72(\mathrm{~s}$, $2 \mathrm{H}), 3.39(\mathrm{~s}, 3 \mathrm{H}), 3.31(\mathrm{~s}, 3 \mathrm{H}), 2.80-2.59(\mathrm{~m}, 5 \mathrm{H}), 2.07-1.80(\mathrm{~m}, 2 \mathrm{H}), 1.63-1.39(\mathrm{~m}, 2 \mathrm{H})$. LRMS (ESI) calculated for $\mathrm{C}_{33} \mathrm{H}_{40} \mathrm{~N}_{9} \mathrm{O}_{2} \mathrm{~S}[\mathrm{M}+\mathrm{H}]^{+}$626.30, found 625.99.

6-((2-methoxy-4-(4-(4-methylpiperazin-1-yl)piperidin-1-yl)phenyl)amino)-4,9-dimethyl-2(trifluoromethyl)-4,9-dihydro-10H-pyrimido[5,4-b]thiazolo[5,4-e][1,4]diazepin-10-one $(33$, BJG-04-035)<smiles>COc1cc(N2CCC(N3CCN(C)CC3)CC2)ccc1Nc1ncc2c(n1)N(C)c1sc(C(F)(F)F)nc1C(=O)N2C</smiles>

General Procedure E was run on $0.05 \mathrm{mmol}$ scale using 2-methoxy-4-(4-(4-methylpiperazin-1yl)piperidin-1-yl)aniline ( $23.1 \mathrm{mg}, 0.076 \mathrm{mmol}, 1.5$ equiv). The reaction mixture was purified by reverse-phase prep HPLC (100-40\% $\left.\mathrm{H}_{2} \mathrm{O} / \mathrm{MeCN}, 20 \mathrm{~mL} / \mathrm{min}, 45 \mathrm{~min}\right)$. Further purification by 
prep TLC (10\% MeOH/2\% NEt $3 / \mathrm{DCM})$ and lyophilization from $\mathrm{H}_{2} \mathrm{O} / \mathrm{MeCN}$ provided the title compound as a yellow solid (11.1 $\mathrm{mg}$ free base).

${ }^{1} \mathrm{H}$ NMR $\left(500 \mathrm{MHz}\right.$, DMSO- $\left.d_{6}\right) \delta 8.38(\mathrm{~s}, 1 \mathrm{H}), 8.26(\mathrm{~s}, 1 \mathrm{H}), 7.60(\mathrm{~d}, J=8.7 \mathrm{~Hz}, 1 \mathrm{H}), 6.64(\mathrm{~d}, J=$ $2.6 \mathrm{~Hz}, 1 \mathrm{H}), 6.52(\mathrm{dd}, J=8.8,2.6 \mathrm{~Hz}, 1 \mathrm{H}), 3.80(\mathrm{~s}, 3 \mathrm{H}), 3.75(\mathrm{~d}, J=12.3 \mathrm{~Hz}, 2 \mathrm{H}), 3.32(\mathrm{~s}, 3 \mathrm{H})$, $2.74-2.62(\mathrm{~m}, 5 \mathrm{H}), 1.91(\mathrm{~s}, 2 \mathrm{H}), 1.67-1.52(\mathrm{~m}, 2 \mathrm{H}) .{ }^{19} \mathrm{~F}$ NMR (471 MHz, DMSO) $\delta-64.35$. LRMS (ESI) calculated for $\mathrm{C}_{28} \mathrm{H}_{35} \mathrm{~F}_{3} \mathrm{~N}_{9} \mathrm{O}_{2} \mathrm{~S}[\mathrm{M}+\mathrm{H}]^{+} 618.26$, found 617.89 .

\section{9-ethyl-6-((2-methoxy-4-(4-(4-methylpiperazin-1-yl)piperidin-1-yl)phenyl)amino)-2,4-} dimethyl-4,9-dihydro-10H-pyrimido[5,4-b]thiazolo[5,4-e][1,4]diazepin-10-one (34, BJG-03180)<smiles>CCN1C(=O)c2nc(C)sc2N(C)c2nc(Nc3ccc(N4CCC(N5CCN(C)CC5)CC4)cc3OC)ncc21</smiles>

General Procedure E was run on $0.05 \mathrm{mmol}$ scale using 2-methoxy-4-(4-(4-methylpiperazin-1yl)piperidin-1-yl)aniline ( $23.1 \mathrm{mg}, 0.076 \mathrm{mmol}, 1.5$ equiv). The reaction mixture was purified by reverse-phase prep HPLC (100-50\% $\left.\mathrm{H}_{2} \mathrm{O} / \mathrm{MeCN}, 20 \mathrm{~mL} / \mathrm{min}, 45 \mathrm{~min}\right)$. Further purification by prep TLC (10\% MeOH/2\% NEt $3 / \mathrm{DCM})$ and lyophilization from $\mathrm{H}_{2} \mathrm{O} / \mathrm{MeCN}$ provided the title compound as a white powder (1.0 mg free base).

${ }^{1} \mathrm{H}$ NMR (500 MHz, DMSO- $\left.d_{6}\right) \delta 8.33(\mathrm{~s}, 1 \mathrm{H}), 8.12(\mathrm{~s}, 1 \mathrm{H}), 7.65(\mathrm{~d}, J=8.7 \mathrm{~Hz}, 1 \mathrm{H}), 6.64(\mathrm{~s}, 1 \mathrm{H})$, $6.51(\mathrm{~d}, J=8.8 \mathrm{~Hz}, 1 \mathrm{H}), 3.83(\mathrm{q}, J=7.0 \mathrm{~Hz}, 2 \mathrm{H}), 3.80(\mathrm{~s}, 3 \mathrm{H}), 3.74(\mathrm{br} \mathrm{s}, 2 \mathrm{H}), 2.67$ (t, $J=12.1$ $\mathrm{Hz}, 2 \mathrm{H}), 2.53$ (s, 3H), $2.02-1.81(\mathrm{~m}, 2 \mathrm{H}), 1.57(\mathrm{br} \mathrm{s}, 2 \mathrm{H}), 1.08(\mathrm{t}, J=7.1 \mathrm{~Hz}, 3 \mathrm{H})$. LRMS (ESI) calculated for $\mathrm{C}_{29} \mathrm{H}_{40} \mathrm{~N}_{9} \mathrm{O}_{2} \mathrm{~S}[\mathrm{M}+\mathrm{H}]^{+} 578.30$, found 577.99. 
4-ethyl-6-((2-methoxy-4-(4-(4-methylpiperazin-1-yl)piperidin-1-yl)phenyl)amino)-2,9dimethyl-4,9-dihydro-10H-pyrimido[5,4-b]thiazolo[5,4-e][1,4]diazepin-10-one (35, BJG-03201-1)<smiles>CCN1c2nc(Nc3ccc(N4CCC(N5CCN(C)CC5)CC4)cc3OC)ncc2N(C)C(=O)c2nc(C)sc21</smiles>

General Procedure E was run on $0.08 \mathrm{mmol}$ scale using 2-methoxy-4-(4-(4-methylpiperazin-1yl)piperidin-1-yl)aniline (34.5 mg, $0.11 \mathrm{mmol}, 1.4$ equiv). The reaction mixture was purified by reverse-phase prep HPLC (100-50\% $\left.\mathrm{H}_{2} \mathrm{O} / \mathrm{MeCN}, 20 \mathrm{~mL} / \mathrm{min}, 45 \mathrm{~min}\right)$. Further purification by prep TLC (10\% $\left.\mathrm{MeOH} / 2 \% \mathrm{NEt}_{3} / \mathrm{DCM}\right)$ and lyophilization from $\mathrm{H}_{2} \mathrm{O} / \mathrm{MeCN}$ provided the title compound as a white powder (4.0 mg free base).

${ }^{1} \mathrm{H}$ NMR $\left(500 \mathrm{MHz}, \mathrm{DMSO}-d_{6}\right) \delta 8.30(\mathrm{~s}, 1 \mathrm{H}), 8.11(\mathrm{~s}, 1 \mathrm{H}), 7.56(\mathrm{~d}, J=8.7 \mathrm{~Hz}, 1 \mathrm{H}), 6.61(\mathrm{~d}, J=$ $2.6 \mathrm{~Hz}, 1 \mathrm{H}), 6.49(\mathrm{dd}, J=8.8,2.6 \mathrm{~Hz}, 1 \mathrm{H}), 3.82-3.75(\mathrm{~m}, 5 \mathrm{H}), 3.72(\mathrm{~d}, J=12.0 \mathrm{~Hz}, 2 \mathrm{H}), 3.27$ (s, 3H), 3.09 (q, $J=7.3 \mathrm{~Hz}, 1 \mathrm{H}), 2.65(\mathrm{t}, J=12.2 \mathrm{~Hz}, 2 \mathrm{H}), 2.53(\mathrm{~s}, 3 \mathrm{H}), 1.96-1.80(\mathrm{~m}, 2 \mathrm{H}), 1.61$ $-1.48(\mathrm{~m}, 2 \mathrm{H}), 1.30(\mathrm{t}, J=7.1 \mathrm{~Hz}, 3 \mathrm{H})$. LRMS (ESI) calculated for $\mathrm{C}_{29} \mathrm{H}_{40} \mathrm{~N}_{9} \mathrm{O}_{2} \mathrm{~S}[\mathrm{M}+\mathrm{H}]^{+} 578.30$, found 578.09 .

6-((2-methoxy-4-(4-(4-methylpiperazin-1-yl)piperidin-1-yl)phenyl)amino)-2,9-dimethyl-4(2,2,2-trifluoroethyl)-4,9-dihydro-10H-pyrimido[5,4-b]thiazolo[5,4-e][1,4]diazepin-10-one (36, BJG-04-091)<smiles>COc1cc(N2CCC(N3CCN(C)CC3)CC2)ccc1Nc1ncc2c(n1)N(CC(F)(F)F)c1sc(C)nc1C(=O)N2C</smiles>

General Procedure E was run on 0.07 mmol scale using 2-methoxy-4-(4-(4-methylpiperazin-1yl)piperidin-1-yl)aniline (32.6 mg, $0.107 \mathrm{mmol}, 1.5$ equiv). The reaction mixture was purified by 
reverse-phase prep HPLC (100-50\% $\left.\mathrm{H}_{2} \mathrm{O} / \mathrm{MeCN}, 20 \mathrm{~mL} / \mathrm{min}, 45 \mathrm{~min}\right)$. Lyophilization from $\mathrm{H}_{2} \mathrm{O} / \mathrm{MeCN}$ provided the title compound as a beige powder (2.5 mg TFA salt).

${ }^{1} \mathrm{H}$ NMR (500 MHz, DMSO- $\left.d_{6}\right) \delta 8.52(\mathrm{~s}, 1 \mathrm{H}), 8.44(\mathrm{~s}, 1 \mathrm{H}), 7.50(\mathrm{~d}, J=8.6 \mathrm{~Hz}, 1 \mathrm{H}), 6.72(\mathrm{~s}, 1 \mathrm{H})$, $6.58(\mathrm{~d}, J=8.5 \mathrm{~Hz}, 1 \mathrm{H}), 4.88$ (br s, 2H), 3.80 (d, $J=7.2 \mathrm{~Hz}, 3 \mathrm{H}), 3.33$ (s, 3H), 2.85-2.72 (m, 7H), $2.56(\mathrm{~s}, 3 \mathrm{H}), 2.08-1.98(\mathrm{~m}, 2 \mathrm{H}), 1.75-1.59(\mathrm{~m}, 2 \mathrm{H}) .{ }^{19} \mathrm{~F}$ NMR (471 MHz, DMSO- $\left.d_{6}\right) \delta-73.58(\mathrm{t}$, $J=9.3 \mathrm{~Hz}$ ). LRMS (ESI) calculated for $\mathrm{C}_{29} \mathrm{H}_{37} \mathrm{~F}_{3} \mathrm{~N}_{9} \mathrm{O}_{2} \mathrm{~S}[\mathrm{M}+\mathrm{H}]^{+} 632.27$, found 632.00 .

6-((2-methoxy-4-(4-(4-methylpiperazin-1-yl)piperidin-1-yl)phenyl)amino)-2,9-dimethyl-4propyl-4,9-dihydro-10H-pyrimido[5,4-b]thiazolo[5,4-e][1,4]diazepin-10-one (37, BJG-04067)

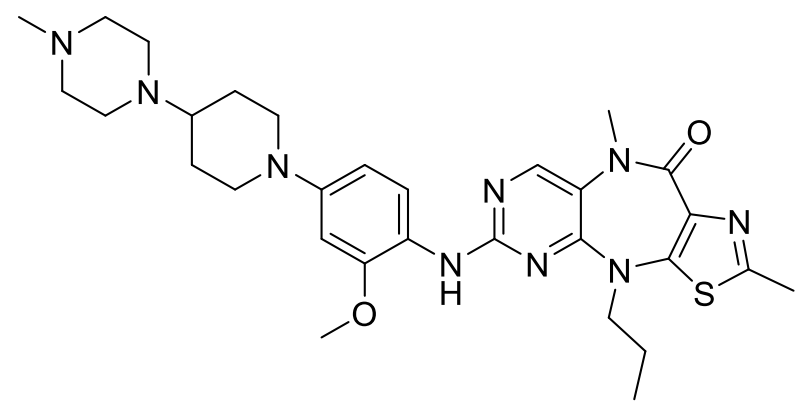

General Procedure E was run on $0.07 \mathrm{mmol}$ scale using 2-methoxy-4-(4-(4-methylpiperazin-1yl)piperidin-1-yl)aniline ( $30.1 \mathrm{mg}, 0.105 \mathrm{mmol}, 1.5$ equiv). The reaction mixture was purified by reverse-phase prep HPLC (100-50\% $\left.\mathrm{H}_{2} \mathrm{O} / \mathrm{MeCN}, 20 \mathrm{~mL} / \mathrm{min}, 45 \mathrm{~min}\right)$. Lyophilization from $\mathrm{H}_{2} \mathrm{O} / \mathrm{MeCN}$ provided the title compound as a white powder (8.0 mg TFA salt).

${ }^{1} \mathrm{H}$ NMR (500 MHz, DMSO- $\left.d_{6}\right) \delta 8.32(\mathrm{~s}, 1 \mathrm{H}), 8.22(\mathrm{~s}, 1 \mathrm{H}), 7.60(\mathrm{~d}, J=8.4 \mathrm{~Hz}, 1 \mathrm{H}), 6.73(\mathrm{~s}, 1 \mathrm{H})$, $6.59(\mathrm{~d}, J=8.8 \mathrm{~Hz}, 1 \mathrm{H}), 3.83-3.77(\mathrm{~m}, 5 \mathrm{H}), 3.73(\mathrm{t}, J=7.2 \mathrm{~Hz}, 2 \mathrm{H}), 3.28(\mathrm{~s}, 3 \mathrm{H}), 2.81(\mathrm{~s}, 3 \mathrm{H})$, $2.53(\mathrm{~s}, 3 \mathrm{H}), 2.04(\mathrm{~d}, J=11.9 \mathrm{~Hz}, 2 \mathrm{H}), 1.77-1.61(\mathrm{~m}, 4 \mathrm{H}), 0.89$ (t, $J=7.3 \mathrm{~Hz}, 3 \mathrm{H})$. LRMS (ESI) calculated for $\mathrm{C}_{30} \mathrm{H}_{42} \mathrm{~N}_{9} \mathrm{O}_{2} \mathrm{~S}[\mathrm{M}+\mathrm{H}]^{+} 592.32$, found 592.09 . 
6-((2-methoxy-4-(4-(4-methylpiperazin-1-yl)piperidin-1-yl)phenyl)amino)-2,4,9-trimethyl4,9-dihydro-10H-oxazolo[5,4-e]pyrimido[5,4-b][1,4]diazepin-10-one (38, BJG-04-016)

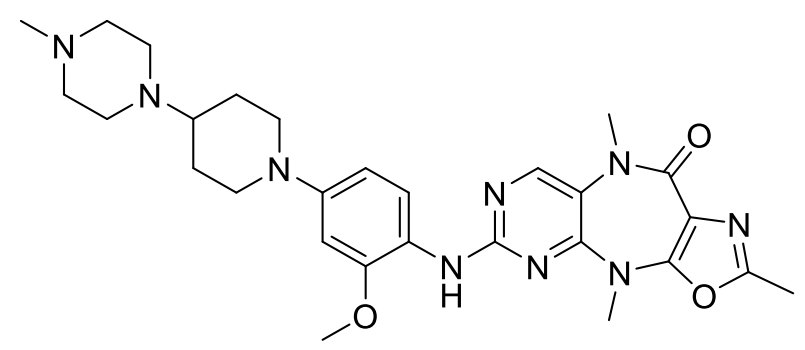

General Procedure E was run on $0.06 \mathrm{mmol}$ scale using 2-methoxy-4-(4-(4-methylpiperazin-1yl)piperidin-1-yl)aniline (23.6 mg, $0.078 \mathrm{mmol}, 1.3$ equiv). The reaction mixture was purified by reverse-phase prep HPLC (100-50\% $\left.\mathrm{H}_{2} \mathrm{O} / \mathrm{MeCN}, 20 \mathrm{~mL} / \mathrm{min}, 45 \mathrm{~min}\right)$. Further purification by prep TLC (10\% $\left.\mathrm{MeOH} / 2 \% \mathrm{NEt}_{3} / \mathrm{DCM}\right)$ and lyophilization from $\mathrm{H}_{2} \mathrm{O} / \mathrm{MeCN}$ provided the title compound as a yellow solid (7.0 $\mathrm{mg}$ free base).

${ }^{1} \mathrm{H}$ NMR (500 MHz, DMSO-d $\left.d_{6}\right) \delta 8.22(\mathrm{~s}, 1 \mathrm{H}), 8.07(\mathrm{~s}, 1 \mathrm{H}), 7.65(\mathrm{~d}, J=8.7 \mathrm{~Hz}, 1 \mathrm{H}), 6.63(\mathrm{~d}, J$ $=2.5 \mathrm{~Hz}, 1 \mathrm{H}), 6.51(\mathrm{dd}, J=8.9,2.6 \mathrm{~Hz}, 1 \mathrm{H}), 3.80(\mathrm{~s}, 3 \mathrm{H}), 3.32(\mathrm{~s}, 6 \mathrm{H}), 3.19(\mathrm{~s}, 3 \mathrm{H}), 2.73-2.61$ (m, 6H), 2.34 (s, 3H), $2.00-1.87$ (br s, 2H), $1.64-1.51$ (br s, 2H). LRMS (ESI) calculated for $\mathrm{C}_{28} \mathrm{H}_{38} \mathrm{~N}_{9} \mathrm{O}_{3}[\mathrm{M}+\mathrm{H}]^{+}$548.31, found 547.99. 


\section{$\underline{\text { Biological Methods }}$}

\section{Cell line experimental procedures}

\section{$\underline{\text { Cell line culturing }}$}

MDA-MB-231 (ATCC) cells were cultured in Dulbecco's Modified Eagle Medium with 10\% FBS and 1\% Penicillin-Streptomycin and HT-29 (ATCC) cells were cultured in McCoy’s 5a Medium with $10 \%$ FBS and 1\% Penicillin-Streptomycin. Cell lines were maintained in $37{ }^{\circ} \mathrm{C} / 5 \% \mathrm{CO}_{2}$ incubators and routinely tested negative for mycoplasma contamination using the MycoAlert Kit (Lonza).

Analysis of MDA-MB-231 and HT-29 cell viability cultured as 2D-adherent monolayers MDA-MB-231 and HT-29 cell viability was assayed in 2D-adherent monolayers using white 384well culture plates (Corning, \#3570) as previously described. ${ }^{13,14}$ In brief, MDA-MB-231 cells were plated at a density of 200 cells per well in $50 \mu \mathrm{L}$ media and allowed to adhere overnight. HT29 cells were plated at a density of 500 cells per well in $50 \mu \mathrm{L}$ media and allowed to adhere overnight. Cells were treated with $100 \mathrm{~nL}$ of compound from compound stock plates using a Janus Workstation pin tool (PerkinElmer) and incubated for $120 \mathrm{~h}$. Cell viability was measured by addition of $10 \mu \mathrm{L}$ of CellTiter-glo (Promega, \#G7570), followed by incubation for 15 minutes at room temperature. Luminescence was measured on an EnVision 2104 Multilabel Plate Reader (PerkinElmer). Data was normalized to DMSO-treated wells and analyzed using GraphPad PRISM v8. 
Analysis of MDA-MB-231 cell viability cultured as ultra-low adherent 3D-spheroids

MDA-MB-231 cell viability was assayed in ultra-low adherent 3D-spheroids using PrimeSurface 384-well 3D culture spheroid plates (S-bio, \#MS-9384WZ) as previously described. ${ }^{14}$ In brief, MDA-MB-231 cells were plated at a density of 500 cells per well in $20 \mu \mathrm{L}$ media with $10 \%$ Matrigel and allowed to form spheroids overnight. Cells were treated with $100 \mathrm{~nL}$ of compound from compound stock plates using a Janus Workstation pin tool (PerkinElmer) and incubated for $120 \mathrm{~h}$. Cell viability was measured by addition of $20 \mu \mathrm{L}$ 3D CellTiter-glo (Promega, \#G9682), followed by incubation for 15 minutes at room temperature. Luminescence was measured on an EnVision 2104 Multilabel Plate Reader (PerkinElmer). Data was normalized to DMSO-treated wells and analyzed using GraphPad PRISM v8.

Analysis of HT-29 cell viability cultured as ultra-low adherent 3D-spheroids

HT-29 cell viability was assayed in ultra-low adherent 3D-spheroids using PrimeSurface 384-well 3D culture spheroid plates (S-bio, \#MS-9384WZ) as previously described. ${ }^{14}$ In brief, HT-29 cells were plated at a density of 500 cells per well in $50 \mu \mathrm{L}$ media and allowed to adhere overnight. Cells were treated with $100 \mathrm{~nL}$ of compound from compound stock plates using a Janus Workstation pin tool (PerkinElmer) and incubated for $120 \mathrm{~h}$. Cell viability was measured by addition of $10 \mu \mathrm{L}$ of CellTiter-glo (Promega, \#G7570), followed by incubation for 15 minutes at room temperature. Luminescence was measured on an EnVision 2104 Multilabel Plate Reader (PerkinElmer). Data was normalized to DMSO-treated wells and analyzed using GraphPad PRISM v8. 


\section{$\underline{\text { Analysis of MDA-MB-231 cell migration }}$}

MDA-MB-231 cells were plated at 1,000,000 cells per $10 \mathrm{~cm}$ plate (Corning, \#0877223) with DMEM containing $10 \% \mathrm{FBS}$ and allowed to adhere overnight in a $37{ }^{\circ} \mathrm{C} / 5 \% \mathrm{CO}_{2}$ incubator. To serum starve cells, culture medium was then replaced with serum-free DMEM for 24 hours. Medium was subsequently removed and cells were dyed with $15 \mu \mathrm{M}$ CellTracker ${ }^{\mathrm{TM}}$ Green CMFDA Dye (Thermo Fisher Scientific, \#C7025) in $3 \mathrm{~mL}$ serum-free DMEM for 45 min in a 37 ${ }^{\circ} \mathrm{C} / 5 \% \mathrm{CO}_{2}$ incubator. Medium was then removed and replaced with $3 \mathrm{~mL}$ serum-free DMEM medium for $30 \mathrm{~min}$. Cells were dissociated with Cell Dissociation Buffer (Thermo Fisher Scientific, \#13151014), pre-treated in suspension in serum-free DMEM with DMSO or indicated

compound, and 25,000 cells per $50 \mu \mathrm{L}$ were seeded into the apical chamber of FluoroBlok ${ }^{\mathrm{TM}}$ 96well cell culture insert (Corning, \#351163). Basolateral chambers were filled with $150 \mu \mathrm{L}$ DMEM containing 10\% FBS (or $150 \mu \mathrm{L}$ serum-free DMEM for chemoattractant-free control samples) and plate was incubated in a $37^{\circ} \mathrm{C} / 5 \% \mathrm{CO}_{2}$ incubator for 24 hours. Fluorescence was measured with a CLARIOstar plate reader (BMG Labtech) and images were acquired with LD A-Plan 5x/0,15 optic (Zeiss, \#421230-9900-000) on Axio Vert.A1 microscope (Zeiss). Background signal acquired from chemoattractant-free control was subtracted from all conditions. Data was normalized to DMSO-treated wells and analyzed using GraphPad PRISM v8.

\section{Gastric organoid experimental procedures}

$\underline{\text { Gastric organoid culturing }}$

$C d h 1^{-/-}$RHOA $^{\mathrm{Y} 42 \mathrm{C} /+}$ gastric organoids were cultured as previously described. ${ }^{15}$ 
Immunoblotting of gastric organoids

$C d h 1^{-/} R H O A^{\mathrm{Y} 42 \mathrm{C} /+}$ gastric organoids were cultured, treated, lysed as previously. ${ }^{15}$ Immunoblotting was performed as previously described ${ }^{16}$ using the following primary antibodies: pFAK Y397 (Cell Signaling, \#3283), FAK (Cell Signaling, \#3285), YAP/TAZ (Cell Signaling, \#8418), active non-phosphorylated YAP1 (Abcam, \#ab205270), pAKT S473 (Cell Signaling, \#4060), AKT (Cell Signaling, \#9272) and $\beta$-Actin (Cell Signaling, \#12620).

Analysis of viability of gastric organoids

$\mathrm{Cdh}^{-/-} \mathrm{RHOA}^{\mathrm{Y} 42 \mathrm{C} /+}$ gastric organoids viability assessment using CellTiter-Glo (Promega, \#G7570) was performed as previously described. ${ }^{15}$ 


\section{Cell Line Supporting Figures}

Supporting Figure 7. FAK dependency in breast, colorectal, and gastric cancer cell lines.
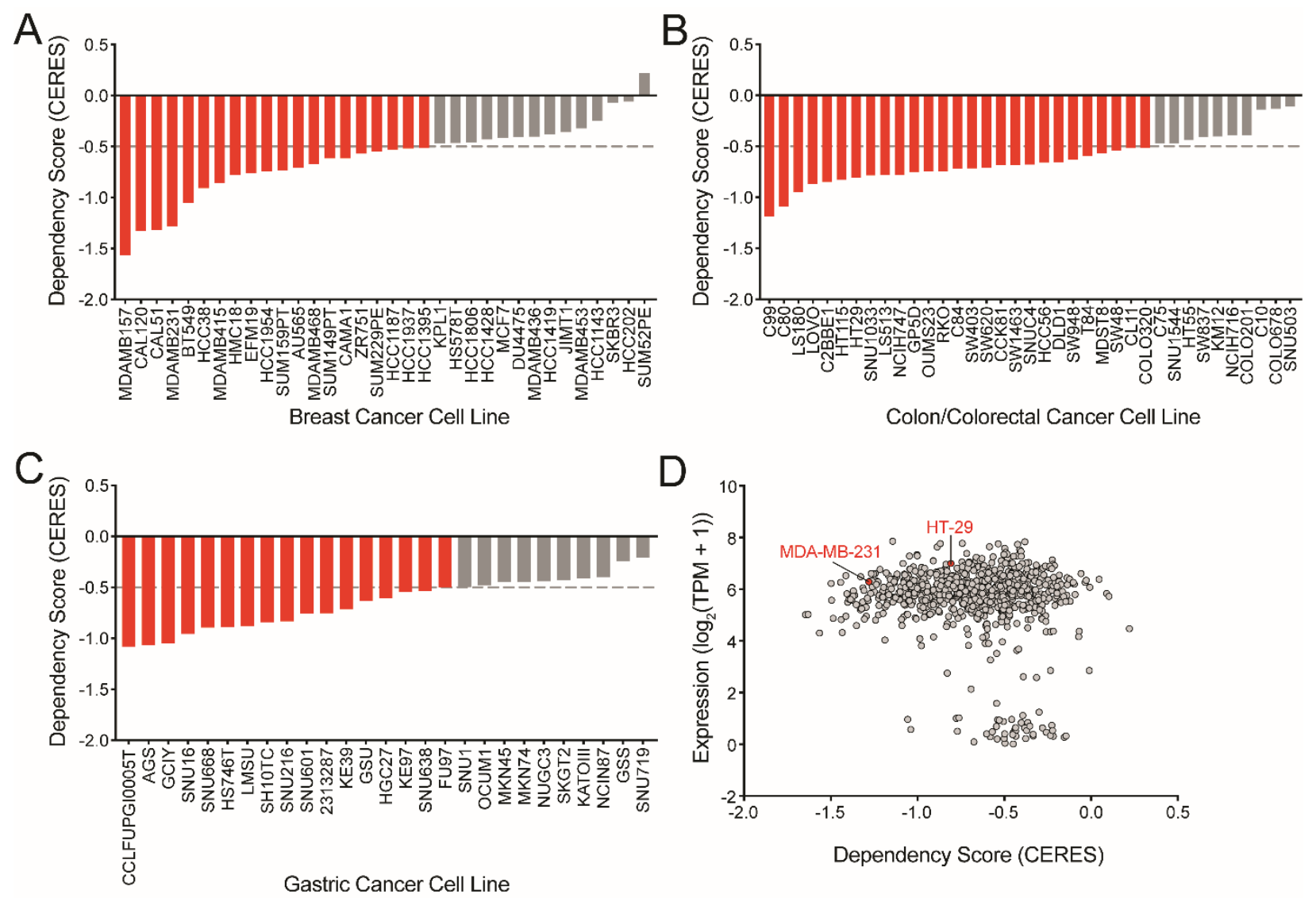

(A-C) PTK2 dependency score for breast (A), colon/colorectal (B), and gastric (C) cancer cell lines. (D) Scatterplot of PTK2 expression versus PTK2 dependency score for all cancer cell lines. Data in A-D is derived the cancer dependency map portal (depmap.org/portal). 
Supporting Figure 8. Evaluation of FAK inhibitors in MDA-MB-231 cells cultured in 2Dmonolayer and 3D-spheroid suspensions.
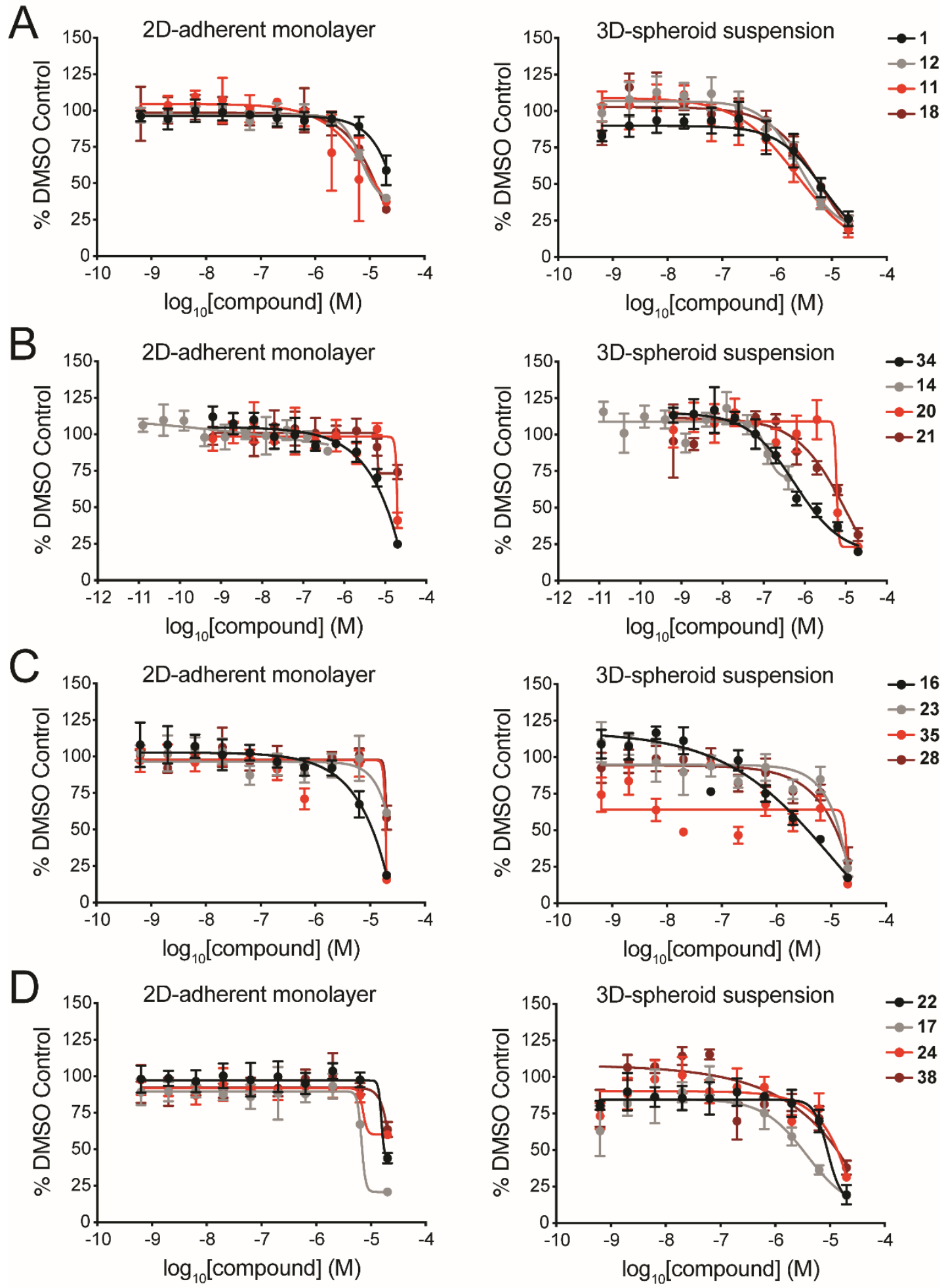
(A-D) DMSO-normalized antiproliferation of MDA-MB-231 cells treated with the indicated compounds for $120 \mathrm{~h}$. Cells were cultured as 2D-adherent monolayers or ultra-low adherent 3Dspheroid suspensions. Data are presented as mean \pm s.d. of $n=4$ biologically independent samples and are representative of $n=3$ independent experiments. 
Supporting Figure 9. Evaluation of FAK inhibitors on migration of breast cancer cells.

A

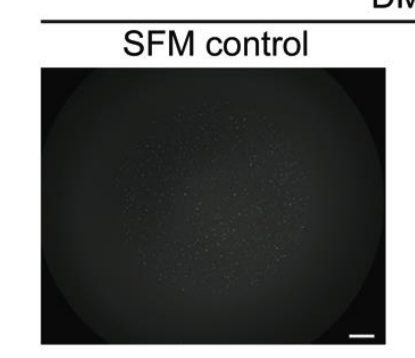

DMSO

B
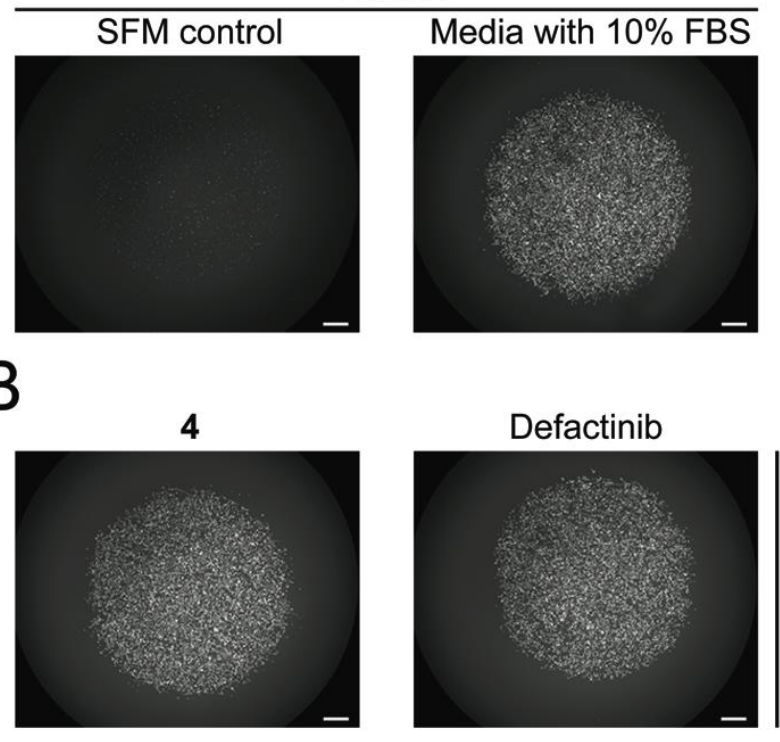

$5 \mu \mathrm{M}$ Nocodazole
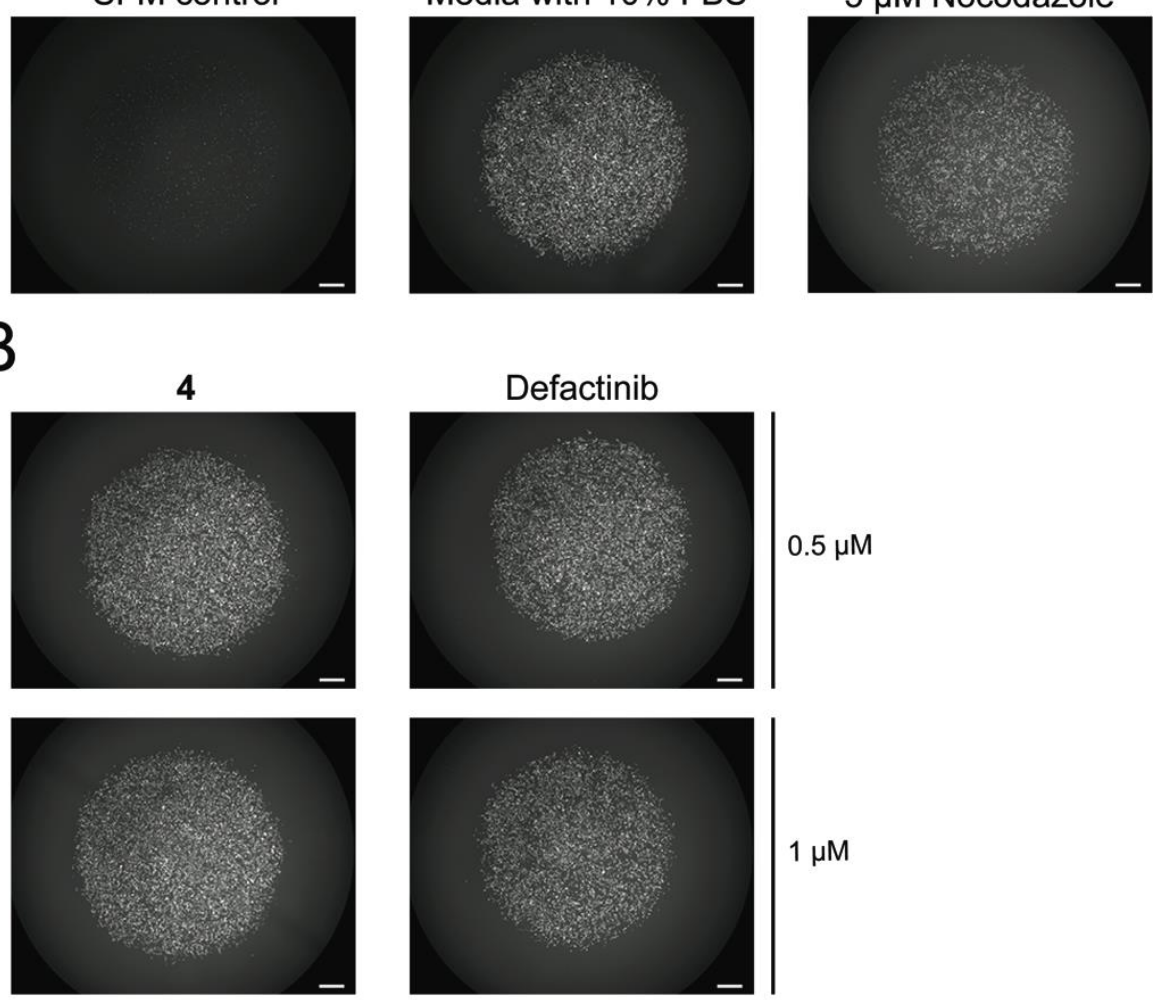

$0.5 \mu \mathrm{M}$
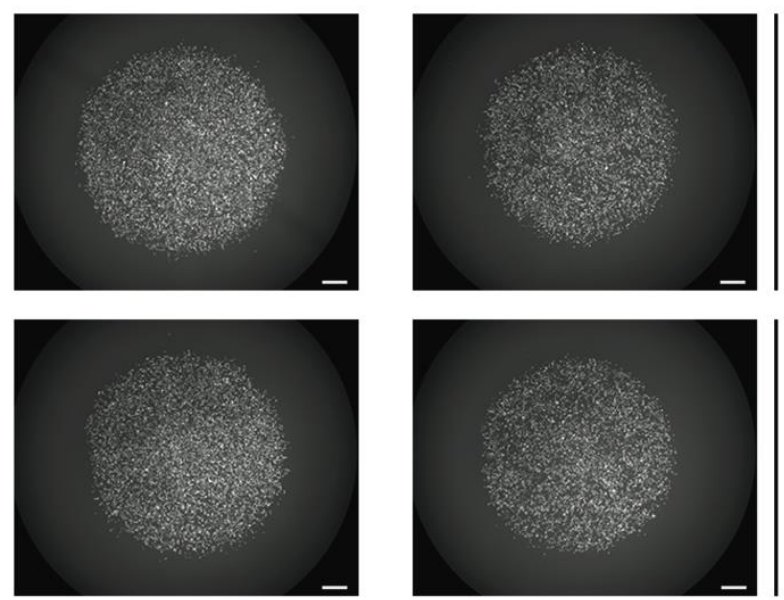

$1 \mu \mathrm{M}$
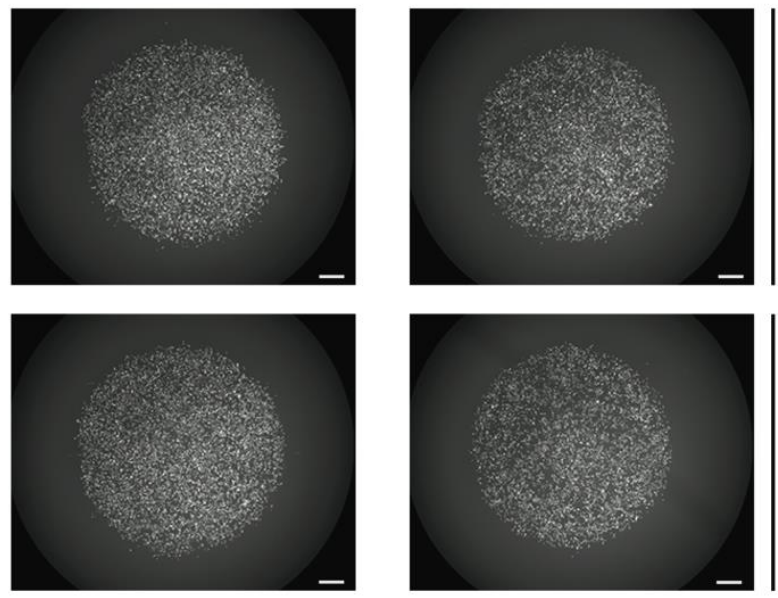

$2.5 \mu \mathrm{M}$
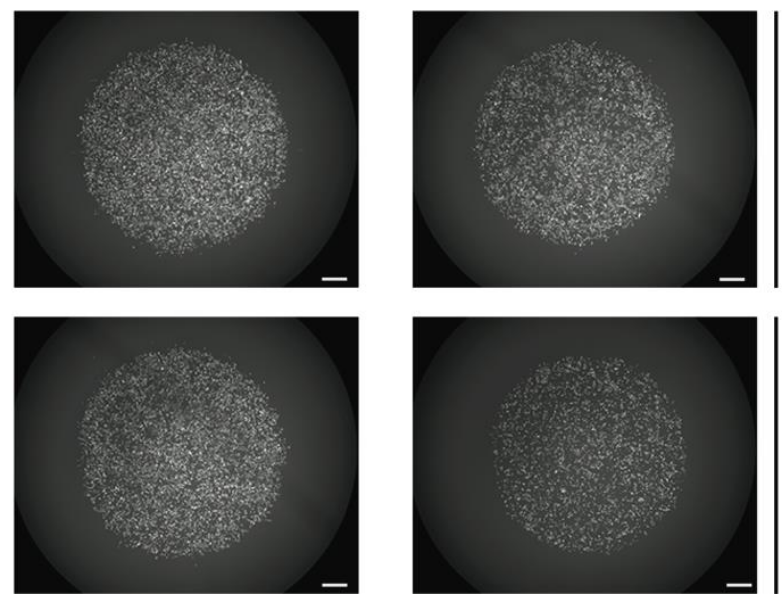

$5 \mu \mathrm{M}$

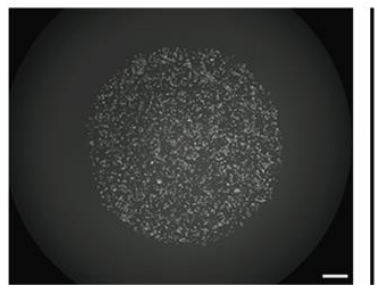

$10 \mu \mathrm{M}$ 
(A-B) Representative images following migration of MDA-MB-231 cells after $24 \mathrm{~h}$ under the indicated conditions. Control conditions are depicted in A (serum free media, SFM). Experimental treatments with $\mathbf{4}$ and defactinib are depicted in B. Images are associated with data presented in Figure 5C. Scale bar represents $400 \mu \mathrm{m}$. 
Supporting Figure 10. Evaluation of FAK inhibitors in HT-29 cells cultured in 2Dmonolayer and 3D-spheroid suspensions.
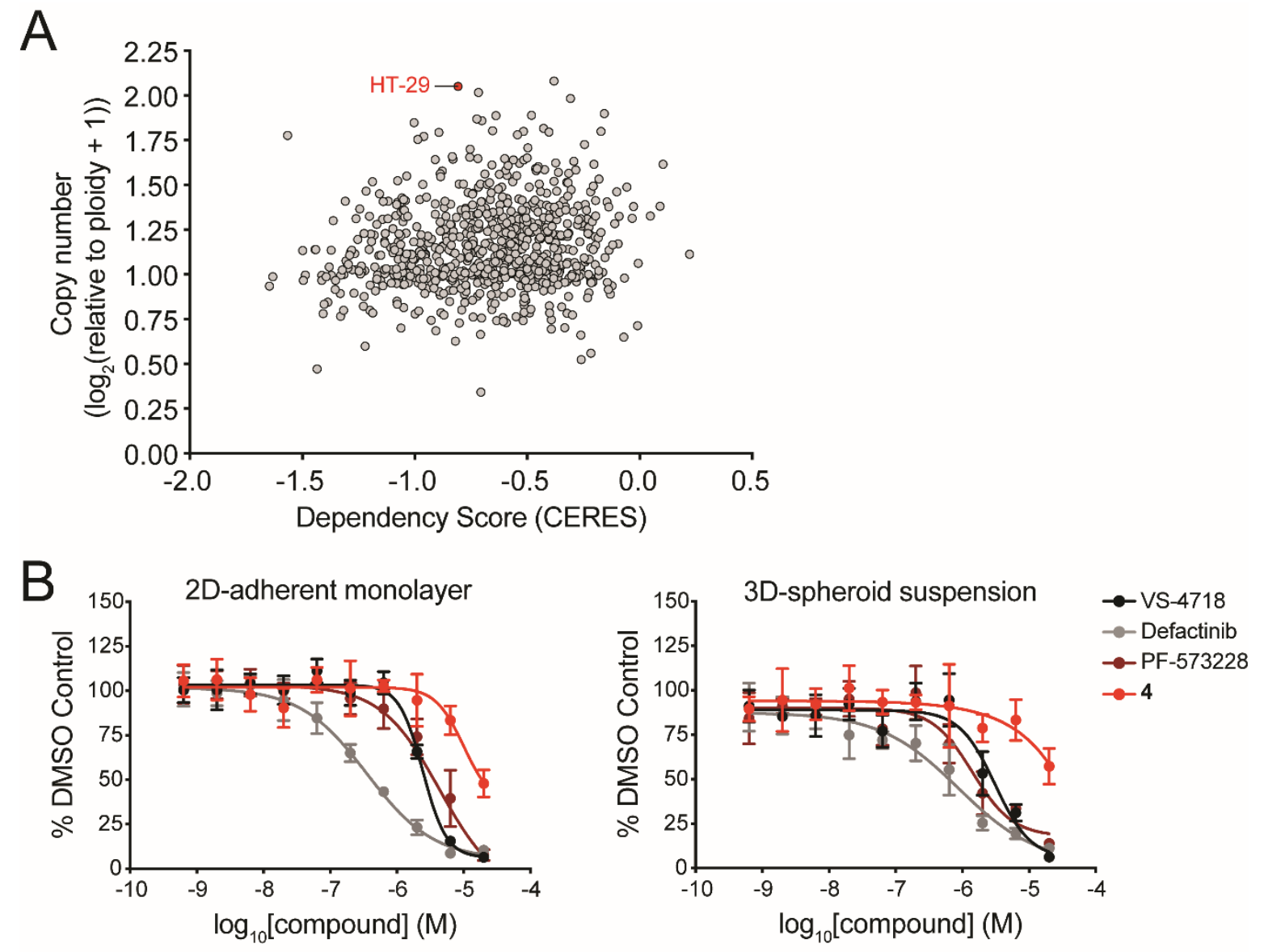

(A) Scatterplot of PTK2 copy number versus PTK2 dependency score for all cancer cell lines. Data is derived the cancer dependency map portal (depmap.org/portal). (B) DMSO-normalized antiproliferation of HT-29 cells treated with the indicated compounds for $120 \mathrm{~h}$. Cells were cultured as 2D-adherent monolayers or ultra-low adherent 3D-spheroid suspensions. Data are presented as mean \pm s.d. of $n=4$ biologically independent samples and are representative of $n=3$ independent experiments. 


\section{Organoid Supporting Figure}

Supporting Figure 11. FAK inhibition upon treatment with 4 decreases YAP and PI3K/AKT signaling of gastric cancer organoids.

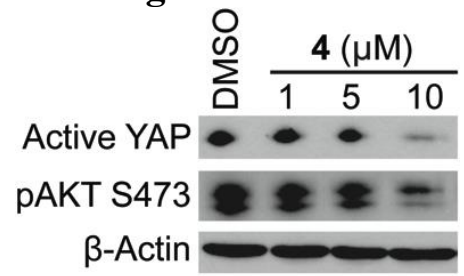

Immunoblot analysis of $C D H 1^{-/-} \mathrm{RHOA}^{\mathrm{Y} 42 \mathrm{C} /+}$ gastric organoids treated with DMSO or 4 at the indicated concentrations for $48 \mathrm{~h}$. Data are representative of $n=3$ independent experiments. 


\section{$\underline{\text { References }}$}

1. Cromm, P. M.; Samarasinghe, K. T. G.; Hines, J.; Crews, C. M., Addressing KinaseIndependent Functions of Fak via PROTAC-Mediated Degradation. J. Am. Chem. Soc. 2018, 140 (49), 17019-17026.

2. Ott, G. R.; Cheng, M.; Learn, K. S.; Wagner, J.; Gingrich, D. E.; Lisko, J. G.; Curry, M.; Mesaros, E. F.; Ghose, A. K.; Quail, M. R.; Wan, W.; Lu, L.; Dobrzanski, P.; Albom, M. S.; Angeles, T. S.; Wells-Knecht, K.; Huang, Z.; Aimone, L. D.; Bruckheimer, E.; Anderson, N.; Friedman, J.; Fernandez, S. V.; Ator, M. A.; Ruggeri, B. A.; Dorsey, B. D., Discovery of Clinical Candidate CEP-37440, a Selective Inhibitor of Focal Adhesion Kinase (FAK) and Anaplastic Lymphoma Kinase (ALK). J. Med. Chem. 2016, 59 (16), 7478-96.

3. Tanjoni, I.; Walsh, C.; Uryu, S.; Tomar, A.; Nam, J. O.; Mielgo, A.; Lim, S. T.; Liang, C.; Koenig, M.; Sun, C.; Patel, N.; Kwok, C.; McMahon, G.; Stupack, D. G.; Schlaepfer, D. D., PND-1186 FAK inhibitor selectively promotes tumor cell apoptosis in threedimensional environments. Cancer Biol. Ther. 2010, 9 (10), 764-77.

4. Roberts, W. G.; Ung, E.; Whalen, P.; Cooper, B.; Hulford, C.; Autry, C.; Richter, D.; Emerson, E.; Lin, J.; Kath, J.; Coleman, K.; Yao, L.; Martinez-Alsina, L.; Lorenzen, M.; Berliner, M.; Luzzio, M.; Patel, N.; Schmitt, E.; LaGreca, S.; Jani, J.; Wessel, M.; Marr, E.; Griffor, M.; Vajdos, F., Antitumor Activity and Pharmacology of a Selective Focal Adhesion Kinase Inhibitor, PF-562,271. Cancer Research 2008, 68 (6), 1935-1944.

5. Chronos Therapeutics Ltd. Micheli, F. B., B.; Gibson, K. R.; Di Fabio, R.; Raveglia, L.; Zanaletti, R.; Cremonesi, S.; Pozzan, A.; Semeraro, T.; Tarsi, L.; Luker, T. J., Substituted 2azabicyclo[3.1.1] heptane and 2-azabicyclo[3.2.1] octane derivatives as orexin receptor antagonists. Patent WO2019/43407 2019.

6. Deng, X.; Yang, Q.; Kwiatkowski, N.; Sim, T.; McDermott, U.; Settleman, J. E.; Lee, J. D.; Gray, N. S., Discovery of a benzo[e]pyrimido-[5,4-b][1,4]diazepin-6(11H)-one as a Potent and Selective Inhibitor of Big MAP Kinase 1. ACS Med. Chem. Lett. 2011, 2 (3), 195-200.

7. Wolfe, J. P.; Åhman, J.; Sadighi, J. P.; Singer, R. A.; Buchwald, S. L., An Ammonia Equivalent for the Palladium-Catalyzed Amination of Aryl Halides and Triflates. Tetrahedron Letters 1997, 38 (36), 6367-6370.

8. Enanta Pharmaceuticals, I. S., B. C.; Kim, I. J.; Blaisdell, T. P.; Yu, J.; Panarese, J.; Or, Y. S., Benzodiazepine Derivatives as RSV Inhibitors. Patent WO2017/015449 2017.

9. Yan, L. H.; Le Roux, A.; Boyapelly, K.; Lamontagne, A. M.; Archambault, M. A.; Picard-Jean, F.; Lalonde-Seguin, D.; St-Pierre, E.; Najmanovich, R. J.; Fortier, L. C.; Lafontaine, D.; Marsault, E., Purine analogs targeting the guanine riboswitch as potential antibiotics against Clostridioides difficile. Eur. J. Med. Chem. 2018, 143, 755-768.

10. Abdel-Magid, A. F.; Carson, K. G.; Harris, B. D.; Maryanoff, C. A.; Shah, R. D., Reductive Amination of Aldehydes and Ketones with Sodium Triacetoxyborohydride. Studies on Direct and Indirect Reductive Amination Procedures. J. Org. Chem. 1996, 61 (11), 3849-3862.

11. Mimura, H.; Kawada, K.; Yamashita, T.; Sakamoto, T.; Kikugawa, Y., Trifluoroacetaldehyde: A useful industrial bulk material for the synthesis of trifluoromethylated amino compounds. Journal of Fluorine Chemistry 2010, 131 (4), 477-486.

12. Montréal., B.-M. S. C. a. U. d.; Banville, J.; Remillard, R.; Ruediger, E. H.; Deon, D. H.; Gagnon, M.; Dubé, L.; Guy, J.; Priestley, E. S.; Posy, S. L.; Maxwell, B. D.; Wong, P. C.; Lawrence, M. R.; Miller, M. M., Imidazothiadiazole and Imidazopyrazine Derivatives as 
Protease Activated Receptor 4 (PAR4) Inhibitors for Treating Platelet Aggregation. Patent WO2013/163279 2013.

13. Nabet, B.; Roberts, J. M.; Buckley, D. L.; Paulk, J.; Dastjerdi, S.; Yang, A.; Leggett, A. L.; Erb, M. A.; Lawlor, M. A.; Souza, A.; Scott, T. G.; Vittori, S.; Perry, J. A.; Qi, J.; Winter, G. E.; Wong, K. K.; Gray, N. S.; Bradner, J. E., The dTAG system for immediate and target-specific protein degradation. Nat Chem Biol 2018, 14 (5), 431-441.

14. Ferguson, F. M.; Nabet, B.; Raghavan, S.; Liu, Y.; Leggett, A. L.; Kuljanin, M.;

Kalekar, R. L.; Yang, A.; He, S.; Wang, J.; Ng, R. W. S.; Sulahian, R.; Li, L.; Poulin, E. J.; Huang, L.; Koren, J.; Dieguez-Martinez, N.; Espinosa, S.; Zeng, Z.; Corona, C. R.; Vasta, J. D.; Ohi, R.; Sim, T.; Kim, N. D.; Harshbarger, W.; Lizcano, J. M.; Robers, M. B.; Muthaswamy, S.; Lin, C. Y.; Look, A. T.; Haigis, K. M.; Mancias, J. D.; Wolpin, B. M.; Aguirre, A. J.; Hahn, W. C.; Westover, K. D.; Gray, N. S., Discovery of a selective inhibitor of doublecortin like kinase 1. Nat. Chem. Biol. 2020.

15. Zhang, H.; Schaefer, A.; Wang, Y.; Hodge, R. G.; Blake, D. R.; Diehl, J. N.; Papageorge, A. G.; Stachler, M. D.; Liao, J.; Zhou, J.; Wu, Z.; Akarca, F. G.; de Klerk, L. K.; Derks, S.; Pierobon, M.; Hoadley, K. A.; Wang, T. C.; Church, G.; Wong, K. K.; Petricoin, E. F.; Cox, A. D.; Lowy, D. R.; Der, C. J.; Bass, A. J., Gain-of-Function RHOA Mutations Promote Focal Adhesion Kinase Activation and Dependency in Diffuse Gastric Cancer. Cancer Discov. 2020, 10 (2), 288-305.

16. Zhang, H. S.; Yan, B.; Li, X. B.; Fan, L.; Zhang, Y. F.; Wu, G. H.; Li, M.; Fang, J., PAX2 protein induces expression of cyclin D1 through activating AP-1 protein and promotes proliferation of colon cancer cells. J Biol Chem 2012, 287 (53), 44164-72. 